\title{
Wnt/PCP-primed intestinal stem cells directly differentiate into enteroendocrine or Paneth cells
}

Anika Böttcher ${ }^{1,2,3}$, Maren Büttner ${ }^{4}$, Sophie Tritschler ${ }^{4,5}$, Michael Sterr ${ }^{1,2,3}$, Alexandra Aliluev ${ }^{1,2,3}$, Lena Oppenländer ${ }^{1,2,3}$, Ingo Burtscher ${ }^{1,2,3}$, Steffen Sass ${ }^{4}$, Martin Irmler ${ }^{3,6}$, Johannes Beckers ${ }^{3,6,7}$, Christoph Ziegenhain $^{8}$, Wolfgang Enard ${ }^{8}$, Andrea C. Schamberger ${ }^{9}$, Fien M. Verhamme ${ }^{9}$, Oliver Eickelberg',

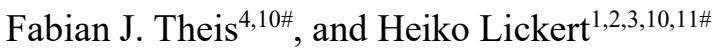

${ }^{1}$ Institute of Diabetes and Regeneration Research, Helmholtz Diabetes Center, Helmholtz Center Munich, 85764 Neuherberg, Germany.

${ }^{2}$ Institute of Stem cell Research, Helmholtz Center Munich, 85764 Neuherberg, Germany.

${ }^{3}$ German Center for Diabetes Research (DZD), 85764 Neuherberg, Germany.

${ }^{4}$ Institute of Computational Biology, Helmholtz Center Munich, 85764 Neuherberg, Germany.

${ }^{5}$ School of Life Sciences Weihenstephan, Technical University of Munich, Freising, Germany.

${ }^{6}$ Institute of Experimental Genetics, Helmholtz Center Munich, 85764 Neuherberg, Germany.

${ }^{7}$ Technical University of Munich, Chair of Experimental Genetics, 85354 Freising, Germany.

${ }^{8}$ Department Biology II, Anthropology and Human Genomics, Ludwig-Maximilians-University of Munich, 82152 Martinsried, Germany.

${ }^{9}$ Comprehensive Pneumology Center, Helmholtz Center Munich, 81377 Munich, Germany.

${ }^{10}$ Technical University of Munich, 80333 Munich, Germany.

${ }^{11}$ Lead contact

\#Authors for correspondence: Heiko Lickert (heiko.lickert@helmholtz-muenchen.de) and Fabian J. Theis (fabian.theis@helmholtz-muenchen.de)

Correspondence and requests for materials should be addressed to H.L.

(heiko.lickert@helmholtz-muenchen.de)

Heiko Lickert, Helmholtz Center Munich, Ingolstaedter Landstraße 1, 85764, Neuherberg, Germany. Phone: +49 8931873760 , FAX: +49 8931873761. 


\section{SUMMARY}

A detailed understanding of intestinal stem cell (ISC) self-renewal and differentiation is required to better treat chronic intestinal diseases. However, different models of ISC lineage hierarchy ${ }^{1-6}$ and segregation ${ }^{7-12}$ are debated. Here we report the identification of $\operatorname{Lgr}^{+}$ISCs that express Flattop (Fltp), a Wnt/planar cell polarity (PCP) reporter and effector gene. Lineage labelling revealed that $\mathrm{Wnt} / \mathrm{PCP}$-activated Fltp ${ }^{+}$ISCs are primed either towards the enteroendocrine or the Paneth cell lineage in vivo. Integration of time-resolved lineage labelling with genome-wide and targeted single-cell gene expression analysis allowed us to delineate the ISC differentiation path into enteroendocrine and Paneth cells at the molecular level. Strikingly, we found that both lineages are directly recruited from ISCs via unipotent transition states, challenging the existence of formerly predicted bi- or multipotent secretory progenitors ${ }^{7-12}$. Transitory cells that mature into Paneth cells are quiescent and express both stem cell and secretory lineage genes, indicating that these cells are the previously described $\mathrm{Lgr}^{+}$labelretaining cells ${ }^{7}$. Wnt/PCP-activated $\mathrm{Lgr}^{+}$ISCs are indistinguishable from Wnt/ $\beta$-cateninactivated $\operatorname{Lgr} 5^{+}$ISCs based on the expression of stem-cell signature or secretory lineagespecifying genes but possess less self-renewal activity. This suggests that lineage priming and cell-cycle exit is triggered at the post-transcriptional level by polarity cues and a switch from canonical to non-canonical Wnt/PCP signalling. Taken together, we identified the Wnt/PCP pathway as a new niche signal and polarity cue regulating stem cell fate. Active Wnt/PCP signalling represents one of the earliest events in ISC lineage priming towards the Paneth and enteroendocrine cell fate, preceding lateral inhibition and expression of secretory lineagespecifying genes. Thus, our findings provide a better understanding of the niche signals and redefine the mechanisms underlying ISC lineage hierarchy and segregation. 
The intestinal epithelium renews continuously throughout life from a pool of $\mathrm{Wnt} / \beta$-catenindependent Lgr5 $^{+}$intestinal stem cells (ISCs) ${ }^{1,13}$. Lineage commitment of Lgr $^{+}$ISCs has been viewed as a binary decision between an absorptive and a secretory progenitor through Notch/Delta-mediated lateral inhibition ${ }^{14,15}$. However, controversies exist regarding the potency of secretory progenitors and their differentiation routes into goblet cells, tuft cells, Paneth cells $(\mathrm{PCs})$ or enteroendocrine cells $(\mathrm{EECs})^{8-12}$. One possibility is that ISCs are functionally heterogeneous and directly differentiate into secretory cell types without passing through proposed stages of bi- or multipotent secretory progenitors. Indeed, functional heterogeneity of ISCs is evident in that i) ISCs positioned at the centre and the periphery of the crypt base produce different clone sizes ${ }^{16}$, ii) reserve stem cells are described at crypt position $+4^{17-20}$ and iii) not all $\operatorname{Lgr}^{+}$cells constitute functional ISCs in vivo ${ }^{21}$ and some might correspond to non-cycling $\mathrm{Lgr}^{+}$label-retaining cells (LRCs), which are PC and/or EEC progenitors ${ }^{7}$. The non-canonical Wnt/planar cell polarity (PCP) pathway controls beta cell differentiation in the pancreas ${ }^{22,23}$ and determines functional heterogeneity in the islets of Langerhans ${ }^{23,24}$. Remarkably, the canonical Wnt/ $\beta$-catenin pathway (which is required for the maintenance of $\mathrm{Lgr5}^{+}$ISCs) and the non-canonical Wnt/PCP pathway share signalling components and antagonize each other's function in some tissues ${ }^{25,26}$. Hence, Wnt/PCP signalling is a prime candidate pathway to control ISC heterogeneity and lineage choice.

\section{Fltp $^{+}$ISCs are fate biased}

To analyse the impact of Wnt/PCP activation on ISC self-renewal and differentiation in the crypt stem cell niche we took advantage of the first reported sentinel for pathway activation, our knock-in Fltp ${ }^{Z V}$ (LacZ $\underline{Z}$ and H2B-VEenus) dual reporter mouse model (Fig. 1a) ${ }^{23,27}$. In this mouse model, Fltp-H2B Venus reporter (FVR) activity is cell-cycle dependent and restricted to quiescent and terminally differentiated cells that had previously induced Fltp expression during Wnt/PCP acquisition ${ }^{23,27}$. When analysing small intestinal (SI) crypt cells in this model, we could distinguish three populations based on their FVR label intensity: FVR ${ }^{\text {neg }}, F R^{\text {low }}$ and FVR $^{\text {hi }}$ cells (Fig. 1b). We isolated these populations using flow cytometry and characterized them using genome-wide transcriptional profiling (Fig. 1c and Extended Data Fig. 1a, b). KEGG pathway analysis revealed that the $\mathrm{FVR}^{\text {neg }}, \mathrm{FVR}^{\text {low }}$ and $\mathrm{FVR}^{\text {hi }}$ populations can be distinguished by their expression of genes involved in absorption, hormone secretion and immune-regulation, respectively, and by genes regulating cell-cycle behaviour and metabolic activity (Extended Data Fig. 1c and Supplementary Table 1). FVR ${ }^{\text {low }}$ and FVR ${ }^{\text {hi }}$ cells were 
highly enriched in transcripts associated with different EEC subsets (Chga, Chgb, Nkx2-2, Neurod1) and the PC lineage (Nupr1, Dll4, Mmp7, Lyz1), respectively, when compared to Lgr5 ${ }^{\text {high }}$ ISCs (Fig. 1c and Extended Data Fig. 1d, e). Further, the FVR ${ }^{\text {hi }}$ population coexpressed ISC markers (Fig. 1c). This combined expression pattern is similar to the transcriptional profile of quiescent $\operatorname{Lgr} 5^{+}$LRCs (Fig. 1c $)^{7}$. The low expression of cell proliferation genes $(C c n d 1, K i 67)$ and the high level of the cell-cycle inhibitor gene Cdkn la in both $\mathrm{FVR}^{+}$populations suggested that $\mathrm{FVR}^{+}$cells undergo terminal differentiation (Extended Data Fig. 1f). Indeed, FVR activity labelled a subset of the postmitotic secretory lineage namely essentially all Lyz1 ${ }^{+}$PCs (95.58\%) and ChgA ${ }^{+}$crypt EECs (96.13\%), but not goblet or tuft cells (Fig. 1d-f and Extended Data Fig. 1g-i). Using a targeted single-cell qRT-PCR approach, we found that Fltp expression was restricted to a few FVR ${ }^{\text {low }}$ EECs and FVR ${ }^{\text {hi }}$ PCs, and to a subset of Lgr5 ${ }^{+}$ISCs (6.2\%) (Fig. 1g). Fltp mRNA expression was induced by Wnt/PCP ligand stimulation indicating that Fltp is also a Wnt/PCP effector in the gut (Fig. 1h). From these data we conclude that i) Fltp is transiently expressed in ISCs, and ii) FVR due to reporter protein stability labels the immediate daughter cells of Fltp ${ }^{+}$ISCs; i.e., more than $95 \%$ of the EECs and PCs.

\section{Wnt/PCP activated Fltp $^{+}$ISCs and Wnt/ $\beta$-catenin activated Lgr5 $^{+}$ISCs are indistinguishable at the transcriptional level}

Homogeneity and equipotency of the ISC pool is vividly discussed ${ }^{3-5,16-19,21,28,29}$. Our data suggested that Fltp ${ }^{+}$ISCs are primed towards the EEC and/or PC lineage. To determine the mechanism underlying ISC heterogeneity and lineage priming, we next used a dual-fluorescent Cre-reporter Fltp ${ }^{T 2 A i C r e /+}$; Gt(ROSA) $26^{m T m G /+}$ mouse line (Extended Data Fig. 2a) ${ }^{30,31}$. In this model, Cre recombinase induces a switch from membrane-Tomato (mT) to membrane-GFP (mG). The intermediate $\mathrm{mTmG}$ state can be captured by flow cytometry and highly expresses Fltp (Fig. 2a-c). In vivo, Fltp ${ }^{+} \mathrm{mTmG}$ cells predominantly located at crypt position $+4 /+5$ (Fig. $2 \mathrm{~d}, \mathrm{e})$. Consistent with the transient expression of the Wnt/PCP reporter gene Fltp in a subset of $\mathrm{Lgr5}^{+}$ISCs (Fig. 1g), the Wnt/PCP activated Fltp ${ }^{+} \mathrm{mTmG}$ cells and $\mathrm{Wnt} / \beta$-catenin activated $\mathrm{Lgr}^{+}$ISCs are closely related as exemplified by similar expression of the $\mathrm{Lgr} 5^{+}$ISC signature genes $^{28}$, lineage-specifying genes and Notch pathway genes, as well as the lack of Lgr $5^{+}$LRC markers $^{7}$ (Fig. 2f-i and Extended Data Fig. 2b, c). However, we found that less mTmG cells were in cell-cycle and formed organoids compared to Lgr $5^{+}$ISCs (Extended Data Fig. 2d-i). As 
chemical injury of the intestine can activate these cells. In contrast to $\mathrm{Wnt} / \beta$-catenin activated $\mathrm{Lgr}^{+}$ISCs, $\mathrm{mTmG}$ cells were resistant to 5-FU treatment but showed reduced mitotic activity (Extended Data Fig. 3a-i). We sporadically detected lineage-traced villi in the small intestine in homeostasis and the number did not increase in 5-FU treated mice indicating that $\mathrm{mTmG}$ cells do not contribute to regeneration after intestinal injury (Extended Data Fig. 3j-1). Together, these data show that Fltp ${ }^{+}$ISCs are transcriptionally similar to $\operatorname{Lgr} 5^{+}$ISCs but possess limited self-renewal capacity in vitro and in vivo. Further, we conclude that Wnt/PCP signallinginduced lineage priming in Fltp ${ }^{+}$ISCs represents the earliest step in the commitment of ISCs towards the PC or EEC lineage. These early cell fate decisions are triggered at the posttranscriptional level by polarity cues and precede Notch/Delta-mediated lateral inhibition.

\section{Intestinal lineages form directly from ISCs via unipotent lineage-specified progenitors}

Secretory lineage specification and in particular the signalling pathways that regulate secretory subtype specification remain poorly understood. Multi-potent secretory progenitors that specify during multiple rounds of division ${ }^{11,15}$, in addition to different lineage-restricted bi-potent secretory progenitors ${ }^{7,9,10,12}$ and direct differentiation from $\mathrm{ISCs}^{2}$, have been proposed (Extended Data Fig. 4a, b). Our data suggested that the PC and EEC lineage directly allocate from ISCs. To elucidate the lineage hierarchy and conclude on lineage relationships in the intestinal epithelium, we made use of the Fltp ${ }^{Z V}$ and Foxa2 Venus Fusion (FVF) reporter mouse lines ${ }^{27,32}$ that label rare intestinal cell populations and performed scRNAseq of 60,000 cells in homeostasis (Fig. 3a, Supplementary Table 2). Using this approach, we could highly enrich for the PC, EEC, goblet and tuft cell lineages (Fig. 3b, c, Extended Data Fig. 4c, d and Supplementary Table 3). Strikingly, due to the enrichment and high resolution of the secretory lineages we could identify all described intestinal epithelial populations and in addition lineagespecified progenitors with proliferative activity and distinct expression profiles (Fig. 3b-e, Extended Data Fig. 4c-g, Extended Data Fig. 5a-c, Supplementary Table 4). Further, to computational reconstruct possible lineage relationships and differentiation trajectories we used partitioned graph abstraction (PAGA) ${ }^{33}$. Strikingly, we found that all lineages originated from ISCs. Progenitor states were highly interconnected reflecting the high plasticity of intestinal epithelial cells ${ }^{34}$ (Extended Data Fig. 5d). Together, these data imply that for every intestinal lineage a progenitor with a distinct transcriptional signature exists and that all lineages directly allocate from ISCs. 


\section{Integration of temporal-resolved lineage labelling with single-cell expression analysis confirms unipotent secretory progenitors}

As transcriptomes alone may not accurately determine the future cell fate of ISCs and progenitors ${ }^{35,36}$ we combined temporal-resolved lineage labelling with a highly sensitive singlecell qPCR approach to fine-map fate decisions towards the PC and EEC lineage (Fig. 4a, b). We determined the expression of 80 well-known and functionally important intestinal signature genes from defined molecular categories; i.e., stem-cell signature genes, lineage markers and determinants, signalling components and cell-cycle regulators (Fig. 4b and Supplementary Table 5). To gain temporal and progenitor state resolution we included: i) Lgr5 ${ }^{\text {hi }}$ ISCs from Lgr5-ki mice, ii) early and late mTmG cells from Fltp ${ }^{T 2 A i C r e /+} ; G t(R O S A) 26^{m T m G /+}$ mice, iii) $\mathrm{FVR}^{+} / \mathrm{Lgr}^{\text {hi }}$ and $\mathrm{FVR}^{+} / \operatorname{Lgr} 5^{\text {low }}$ double-positive cells from $\mathrm{Fltp}^{Z V} / \mathrm{Lgr} 5$-ki dual reporter mice (Fig. 4c-e), and iv) Neurog3-expressing EEC progenitors from Ngn3-Venus mice ${ }^{37}$. UMAP visualization of the single-cell qPCR data showed that the flow-sorted FVR ${ }^{\text {hi }}$ PCs grouped into one defined cluster, whereas the FVR ${ }^{\text {low }}$ EECs grouped into two clusters (Fig. 4f). Early mTmG cells grouped together with $\mathrm{Lgr} 5^{\mathrm{hi}}$ ISCs and late $\mathrm{mTmG}$ cells grouped together with a subset of FVR $^{\text {low }}$ cells (Fig. 4f).

To delineate the ISC differentiation path into the EEC and PC lineages we used PAGA ${ }^{33}$. Ordering of cells along a pseudotime as a proxy for real-time differentiation identified three terminal states from our single-cell qPCR snapshot data: the PC branch with terminal state 1, the EEC branch with terminal state 2 and a third branch with unassigned cells (Fig. 4g-j and Extended Data Fig. 6a). Separation into the EEC and PC lineages occurred early and still within the ISC population, which reinforces that EECs and PCs directly allocate from $\mathrm{Lgr}^{+}$ISCs (Fig. 4i, j and Extended Data Fig. 6b). Plotting gene expression versus pseudotime revealed that cells differentiate via lineage-specific unipotent transition states characterized by downregulation of stem cell markers for the EEC branch and co-expression of stem-cell and secretory markers for the PC branch (Extended Data Fig. 6b-f). Unassigned cells were mainly late mTmG and FVR ${ }^{\text {low }}$ cells (Fig. 4j) and did not express mature EEC or PC markers (Extended Data Fig. 6a), but instead were characterized by the expression of genes implicated in cell-matrix adhesion and signalling (Itgal, ItgaV), repression of endocrine cell fate (Gfil) and regulation of Notch signalling (Lfng) (Extended Data Fig. 6b). Together, the pseudotemporal analysis further supports our realtime lineage reporter-based finding that PCs and EECs directly allocate from ISCs via unipotent progenitors. 


\section{FVR $^{+}$LRCs differentiate into PCs}

Single-cell transcriptomics data suggest that PCs are mainly formed via a PC/goblet cell precursor and that only a small subset directly differentiates from $\mathrm{ISCs}^{2}$. However, our integrated analysis of lineage labelling with single-cell gene expression indicates that in addition to EECs most of the PCs also directly allocate from ISCs via a unipotent transition state comprising mainly Lgr5 ${ }^{+} / \mathrm{FVR}^{+}$double-positive cells (Fig. 4j and Extended Data Fig. 6).

Using the FVR mice we obtained transcriptional profiles of more than 20,000 cells from the rare and difficult to capture PC lineage ${ }^{29}$ (Fig. 3). When we investigated the transition of ISCs to PCs we identified a ISC population that connects to the PC progenitor population, which we termed PC-primed ISCs (Fig. 5a). In addition, we found two mature PC types which differ in the expression of $L y z 2$ (Fig. 5b). Pseudotemporal ordering of ISCs and PC subclusters placed the PC progenitor in between the mature PCs and ISCs, while PC-primed ISCs link to PC progenitors (Fig. 5c). The co-expression of stem-cell and secretory lineages genes in the PC progenitor suggested that these are the recently described quiescent $\operatorname{Lgr}^{+}$LRCs $^{7}$ (Fig. 4, 5c, d and Extended Data Fig. 6a-c). With the exception of PCs that have a lifespan of about 6-8 weeks, all other differentiated intestinal lineages are renewed every 4-5 days. Quiescent or slowly cycling intestinal cells persist for more than 10 days and thus are defined by the property of label-retention. To assess label-retention within the $\mathrm{FVR}^{+}$population we "birth-dated" this population with 5-bromodeoxyuridine (BrdU) (Fig. 5e-g). After a chase period of 10 days, 30\% of the $\mathrm{FVR}^{+}$cells retained the label and were non-PCs (Lyz1 ${ }^{-}$), and hence LRCs (Fig. 5h). After a chase period of 21 days, all $\mathrm{BrdU}^{+}$LRCs present in the crypt were also $\mathrm{FVR}^{+}$. An increase in $\mathrm{FVR}^{+} / \mathrm{BrdU}^{+} / \mathrm{Lyz}^{+}$cells implied that $\mathrm{FVR}^{+}$LRCs primarily give rise to PCs (Fig. 5h). Like Fltp $^{+}$ISCs (mTmG cells), $\mathrm{FVR}^{+}$LRCs were predominantly located at position $+4 /+5$ (Fig. $2 \mathrm{~d}$, e and Fig. 5i-k). These results indicate that PCs directly arise from ISCs via a quiescent, labelretaining transition state, which is characterized by co-expression of stem cell and secretory lineage markers. The identification of lineage-specific unipotent transition states together with the fact that FVR labels more than 95\% of all PCs and EECs, but not goblet or tuft cells challenges the existence of formerly predicted bi- or multipotent secretory progenitors.

\section{Wnt/PCP signalling is activated during PC and EEC differentiation}

Fltp is transiently expressed in ISCs that acquired Wnt/PCP activation and are committed to differentiate into PCs and EECs (Fig. 1g, Fig. 2f, g). Cell ordering along the pseudotime 
confirmed that i) Wnt/PCP signalling is specifically activated in ISCs that differentiate towards PCs and EECs indicated by upregulation of several Wnt/PCP genes such as Vangl2, Dvl2, Ror2 and Celsrl and ii) Wnt/PCP pathway activation precedes Notch/Delta-mediated lateral inhibition and cell-cycle exit (Extended Data Fig. 7a, b and Extended Data Fig. 2b). Consistent with the expression of core pathway components we also detected increased expression of Wnt/PCP pathway genes and Jnk activity in the $\mathrm{FVR}^{+}$population, indicative of active Wnt/PCP signalling (Extended Data Fig. 7c-f). To further corroborate a role of Wnt/PCP signalling in cell fate regulation we analysed Celsr ${ }^{\mathrm{crsh} /+} ; F{ }^{2}{ }^{Z V / Z V}$ compound mutant mice. Celsr1 is a core Wnt/PCP component and member of a family consisting of Celsr1-3. Gene expression analysis of 14,000 mutant cells (from $n=4$ mutant mice) revealed a less proliferative PC progenitor population and aberrant gene expression of known secretory lineage regulators (e.g. Sox9, Atoh1, Spdef, Foxa3, Tead2 and Jun) and potentially new regulators (e.g. Ybx1, Pa2g4, Hnrnpk) as well as canonical Wnt target genes specifically in the PC lineage suggesting disturbances in the differentiation of PCs (Extended Data Fig. 8a-e). Assessment of PC and EEC numbers showed a slight reduction of PCs when compared to control mice (Extended Data Fig. 8f, g). These weak disturbances in gene expression and PC and EEC numbers in this mutant mouse model is most likely due to functional redundancy of Wnt/PCP proteins during PC and EEC differentiation (Extended Data Fig. 7).

Taken together, we identified the Wnt/PCP pathway as a new niche signal that determines stem cell fate. We propose that a switch from $\mathrm{Wnt} / \beta$-catenin to $\mathrm{Wnt} / \mathrm{PCP}$ signalling induces PC and EEC lineage priming and cell-cycle exit of ISCs. This is consistent with our recent findings that Wnt/PCP activation triggers functional maturation and cell-cycle exit of endocrine insulinproducing $\beta$-cells in the pancreatic islet ${ }^{23}$ and suggests that polarity cues regulate cell heterogeneity and terminal differentiation in the crypt and islet cell niche. 


\section{Methods and Supplementary material}

Animal studies. Animal experiments were carried out in compliance with the German Animal Protection Act and with the approved guidelines of the Society of Laboratory Animals (GVSOLAS) and of the Federation of Laboratory Animal Science Associations (FELASA). This study was approved by the institutional Animal Welfare Officer and by the Government of Upper Bavaria, Germany.

Mouse lines used:

Fltp ${ }^{Z V}(\mathrm{C} 57 \mathrm{BL} / 6 \mathrm{~J})^{27}$, Fltp ${ }^{T 2 A i C r e}$ (mixed C57BL/6J, CD1 background) ${ }^{30}$ crossed with Gt(ROSA)26 $6^{m T m G}$ (mixed 129/SvJ, C57BL/6J background) ${ }^{31}$, Lgr5-EGFP-IRES-creERT2 $(\mathrm{C} 57 \mathrm{BL} / 6 \mathrm{~J})^{1}, \mathrm{Celsr}^{\mathrm{Crsh}}$ (mixed BALB/c, C57BL/6J background) ${ }^{38}$, Ngn3-VF (mixed 129/SvJ, $\mathrm{C} 57 \mathrm{BL} / 6 \mathrm{~J}$ background $)^{37}$, homozygous Foxa2-Venus fusion (FVF) ${ }^{39}$ mice were generated as previously described and backcrossed to $\mathrm{C} 57 \mathrm{BL} / 6$ background for at least 10 generations.

All experiments were performed using 3-6-month-old mice, unless indicated otherwise.

\section{Tissue preparation and immunohistochemistry}

The intestine was isolated and flushed with ice-cold PBS, fixed with 4\% paraformaldehyde (PFA) for $3 \mathrm{~h}$ at $4{ }^{\circ} \mathrm{C}$ and then placed for cryoprotection in a progressive sucrose gradient $(7.5 \%$ sucrose for $1 \mathrm{~h}, 15 \%$ sucrose for $1 \mathrm{~h}, 30 \%$ sucrose overnight). Tissue was embedded in Optimum

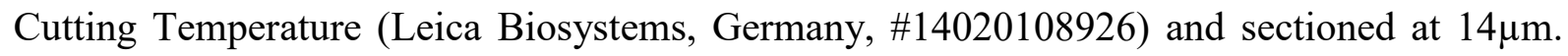
Isolated intestinal crypts were fixed for whole-mount stainings with 4\% PFA for 30min at room temperature (RT). After fixation crypts were washed three times with PBS. For immunofluorescence staining, sections or isolated crypts were permeabilized with $0.5 \%$ Triton $\mathrm{X}-100$ in PBS for $30 \mathrm{~min}$ at RT, blocked [(10\% FCS, $0.1 \%$ BSA and 3\% donkey serum in PBS/0.1\%Tween-20 (PBST)] for $1 \mathrm{~h}$ and incubated with primary antibodies overnight at $4{ }^{\circ} \mathrm{C}$. Sections or crypts were washed in PBST, incubated with secondary antibodies in blocking solution for $1 \mathrm{~h}$ at RT, followed by a DAPI (ROTH, 6335.1) staining to visualize the nuclei and mounted with the Elvanol antifade reagent.

For BrdU staining tissue was sectioned at $14 \mu \mathrm{m}$ and stained according to standard procedure followed by incubation in $3.3 \mathrm{~N} \mathrm{HCl}$ for $10 \mathrm{~min}$ on ice, $50 \mathrm{~min}$ at $37^{\circ} \mathrm{C}$ and incubation with borate buffer $\mathrm{pH} 8.5$ for $2 \mathrm{x} 15 \mathrm{~min}$ at $\mathrm{RT}$.

For stainings on single cells (Ki67, pJnk, EdU, Venus) cells were isolated by flow cytometry and cytospun on glass slides. Cells were dried and fixed with $4 \%$ PFA for 10min at RT, permeabilized with $0.25 \%$ Triton-X100 in PBS for $15 \mathrm{~min}$ at RT and then blocked for $1 \mathrm{~h}$ at RT 
followed by an overnight incubation with the primary antibody. Cells were washed in PBST, incubated with secondary antibodies in blocking solution for $1 \mathrm{~h}$ at RT, followed by a DAPI (ROTH, 6335.1) staining to visualize the nuclei and mounted with the Elvanol antifade reagent. Sections, cells and crypts were visualized using a Leica SP5 confocal microscope.

The following primary antibodies were used for immunofluorescent stainings: chicken antiGFP (1:600, Aves Labs, USA, GFP-1020); rat anti-BrdU (1:200, Abcam, ab6326); rat anti-RFP (1:500, Chromotek, ORD003515), goat anti-ChgA (1:200, Santa Cruz, sc-1488); rabbit antiLyz1 (1:1000, DAKO, A0099); rabbit anti-Muc2 (1:500, Santa Cruz, sc-7314); rabbit antiDclk1 (1:200, Abcam, ab37994); rat anti-BrdU (1:200, Abcam, ab6326); rabbit anti-Ki67 (1:200, Abcam, ab15580); rabbit anti-pJnk (1:100, NEB, \#4668). The following secondary antibodies were used: donkey anti-chicken Alexa Fluor 488 (Dianova, 703-225-155); donkey anti-mouse Cy5 (Dianova, 715-175-151); donkey anti-goat Alexa Fluor 555 (Invitrogen, A21432); donkey anti-rabbit Alexa Fluor 555 (Invitrogen, A31572); donkey anti-rabbit Alexa Fluor 649 (Dianova, 711-605-152); donkey anti-rat DyLight 549 (Dianova, \#712-505-153).

\section{pJnk fluorescence intensity analysis}

Lgr5 $^{\text {hi }}$ and $\mathrm{mTmG}$ cells were isolated by flow cytometry and cytospun on glass slides. Cells were stained and imaged using a Leica SP5 Confocal microscope. Analysis was performed using the Leica LAS-AF (Version 2.7.3.9723) software. pJnk fluorescent intensity signal was determined from each cell and background signal was subtracted (secondary antibody only).

\section{BrdU pulse-chase experiment}

$1 \mathrm{mg} / \mathrm{ml} \mathrm{BrdU}$ (Sigma, \#B5002), in combination with 1\% sucrose, was administered to mice via their drinking water for 14 days. BrdU-containing drinking water was exchanged every three days. Mice were sacrificed after 14 days of continuous BrdU labelling to assess the initial labelling efficiency and after a chase period of 10 and 21 days to assess label retention. Intestines were removed, flushed with ice-cold PBS, fixed with $4 \%$ PFA for $3 \mathrm{~h}$ at $4{ }^{\circ} \mathrm{C}$, cryoprotected by a sucrose gradient and embedded in OCT. Cryosection imaging was performed using a Leica SP5 confocal microscope and cells were counted manually.

\section{5-FU treatment}

Intestinal injury was induced by injecting two intraperitoneal doses of 5-fluorouracil (5-FU, $100 \mathrm{mg} / \mathrm{kg}$, Sigma) over a $48 \mathrm{~h}$ period. Mice were sacrificed $48 \mathrm{~h}$ or 26 days after the last 5-FU dose and intestinal tissue was analyzed by immunohistochemistry and FACS. To assess the 
replication rate in the small intestine, 5-Ethynyl-2-deoxyuridine (EdU) (Thermo Fisher Scientific, A10044) was administered as an i.p. injection at $100 \mu \mathrm{g} / \mathrm{g}$ body weight from a 10 $\mathrm{mg} / \mathrm{ml}$ stock in sterile PBS. Mice were sacrificed $4 \mathrm{~h}$ post EdU administration. Lgr ${ }^{\text {hi }}$ and $\mathrm{mTmG}$ cells were isolated by flow cytometry and cytospun on glass slides. EdU staining was performed on cytopun cells using the Click-iT Staining Kit (Invitrogen, \#C10340) according to the manufacturer's instructions.

\section{Western blot analysis}

For Western blot analysis, $\mathrm{FVR}^{+}$and $\mathrm{FVR}^{-}$cells were FAC-sorted and lysed in RIPA buffer (50mM Tris pH7.5, 150mM NaCl, 1mM EDTA, 1\% Igepal, 0.1\% SDS, 0.5\% Sodiumdeoxycholate) containing phosphatase inhibitor (Sigma-Aldrich, P5726, P0044) and proteinase inhibitor (Sigma-Aldrich, P8340). Cell lysates were resolved by SDS-PAGE, transferred to PVDF membrane (Biorad) and incubated with the following primary antibodies: rabbit antipJnk (1:1000, NEB, \#4668); rabbit anti-Jnk (1:1000, NEB, \#9258); mouse anti-Gapdh (Merck Biosciences, CB1001); rabbit anti mTor (Cell Signaling, \#2972); rabbit anti-pmTor (Ser2448) (Cell Signaling, \#5536). Protein bands were visualized using horseradish peroxidase (HRP)conjugated antibody, goat anti-mouse HRP (Dianova, 115-036-062) or goat anti-rabbit HRP (Dianova, 111-036-045) and chemiluminescence reagent (Millipore). The bands were quantified using ImageJ.

\section{Flow cytometry}

For gene expression (microarray, single-cell RNAseq/qRT-PCR, qRT-PCR) analysis, Western Blot, and single-cell culture, crypt cells were sorted using FACS-Aria III (BD Bioscience) with a $100 \mu \mathrm{m}$ nozzle. For all experiments, single cells were gated according to their FSC-A (front scatter area) and SSC-A (side scatter area). Singlets were gated dependent on the FSC-W (front scatter width) and FSC-H (front scatter height) and dead cells were excluded using the 7-AAD marker (eBioscience, \#00-6993-50).

\section{Crypt isolation, crypt culture in matrigel, single-cell preparation for FACS, intestinal single-cell culture}

Isolation and culture of small intestinal crypts and organoid culture was performed as previously described ${ }^{40}$. Briefly, intestines were harvested and washed with PBS. Villi were scraped away using coverslips. The remaining tissue was cut into $2 \mathrm{~cm}$ pieces and incubated in $2 \mathrm{mM}$ EDTA/PBS for $35 \mathrm{~min}$ at $4^{\circ} \mathrm{C}$. Finally, crypts were collected by shaking. For Wnt- 
stimulation, isolated crypts were cultured in growth factor-free matrigel (BD Bioscience \#356231) overlaid with medium containing 50ng/ml EGF (Life technologies PMG8043)/100ng/ml mNoggin (Peprotech, \#250-38)/1 $\mu \mathrm{g} / \mathrm{ml} \mathrm{mR}$-spondin1 (R\&D sytems, \#2474-RS-050) (ENR) in the presence of 10 $\mu \mathrm{M}$ Rock-inhibitor (Sigma, Y0503). Crypts were plated in 24-well plates at a density of 400 crypts/40 $\mu 1$ matrigel. Two days after plating, the medium was changed for Wnt stimulation to ENR containing 400ng/ml Wnt ligand (Wnt5a, R\&D systems \#645-WN-010; Wnt11, R\&D systems \#6179-WN-010). After 2 days culture with Wnt ligands, crypts were intensively washed with ice-cold PBS and lysed in QIAzol (Qiagen, \#79306) for RNA isolation (Qiagen, \#79306) and cDNA synthesis (Invitrogen, SuperScript VILO cDNA synthesis kit, \#11754).

For single-cell preparation, the crypt pellet was resuspended in 1-1,5ml TrypLE (Life technologies, \#12605), incubated on ice for $5 \mathrm{~min}$, followed by $5 \mathrm{~min}$ incubation at $37^{\circ} \mathrm{C}$ in a water bath. Then, $6 \mathrm{ml}$ of crypt complete medium containing $10 \%$ FCS and $10 \mu \mathrm{g} / \mathrm{ml}$ DNase were added, and cells were incubated for $5 \mathrm{~min}$ at $37^{\circ} \mathrm{C}$ in a water bath. The cells were gently resuspended by pipetting up and down 10 times, $10 \mathrm{ml} \mathrm{FACS}$ buffer ( $2 \%$ FCS, $2 \mathrm{mM}$ EDTA in PBS) was added and the cells were centrifuged at $300 \mathrm{xg}, 5 \mathrm{~min}, 4^{\circ} \mathrm{C}$. Cells were washed twice with FACS buffer and finally the cell pellet was re-suspended in 1-2ml FACS buffer containing $10 \mu \mathrm{M}$ Rock-inhibitor (Sigma, Y0503), and cells were passed through the $40 \mu \mathrm{m}$ cell strainer caps of FACS tubes.

Single-cell culture to assess organoid formation efficiency, was performed as described previously ${ }^{41}$. 6,000 cells/25 $\mu 1$ matrigel (BD Bioscience \#356231) were seeded in a 24-well and overlaid with medium containing ENR, 10 $\mu$ M Rock-inhibitor (Sigma, Y0503), 1mM Valproic

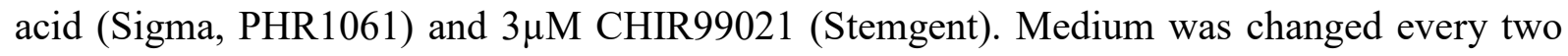
days. VPA and CHIR99021 were added for the first 6 culture days. Bright-field images were acquired using a Zeiss microscope. Organoids were counted after 12 days culture.

\section{RNA isolation, qRT-PCR and microarray mRNA profiling}

For gene profiling and qRT-PCR cells were directly sorted into Qiazol lysis reagent (Qiagen, \#79306) and total RNA was extracted using the miRNeasy Micro kit (Qiagen, \#217084), RNA integrity was checked using Agilent 2100 Bioanalyzer (Agilent RNA 6000 Pico Kit) and cDNA was amplified with the Ovation PicoSL WTA System V2 in combination with the Encore Biotin Module (Nugen, USA). Amplified cDNA was hybridized on Affymetrix Mouse Gene 1.0 ST arrays (FVR) or Affymetrix Mouse Gene 2.0 ST arrays ( $\left.\mathrm{Lgr}^{\mathrm{hi}} / \mathrm{mTmG}\right)$. Staining and scanning was performed according to the Affymetrix expression protocol, including minor modifications 
as suggested in the Encore Biotin protocol. For FVR data Expression Console (v.1.3.0.187, Affymetrix) was used for quality control and to obtain annotated normalized RMA gene-level data (standard settings including median polish and sketch-quantile normalisation). $\mathrm{Lgr}^{\text {hi }} / \mathrm{mTmG}$ data were RMA normalized using R/Bioconductor package oligo (version 1.38.0) and probesets were annotated using the R/Bioconductor package mogene20sttranscriptcluster.db (version 8.5.0). Differential expression analyses were performed with the $\mathrm{R}$ environment for statistical computing ( $\mathrm{R}$ Development Core Team, http://www.R-project.org/) by using the limma package (version 3.30.7) and P-values were adjusted for multiple testing by Benjamini-Hochberg correction. A gene was considered as differentially expressed if the adjusted p-value (FDR) was below a threshold of 0.05 (for FVR) or $<0.01$ (for $\mathrm{Lgr}^{\mathrm{hi}} / \mathrm{mTmG}$; an additional filter for fold-change $>2 \mathrm{x}$ was applied). Heatmaps were drawn using the pheatmap library for R. Expression is scaled row-wise and the colours range from dark blue (low expression) to orange (high expression). Functional enrichments were conducted using the GOstats package for R.

\section{TaqMan qRT-PCR}

TaqMan qRT-PCR was performed under standard conditions using ViiA7 (Applied Biosystems) and TaqMan Fast Advanced Master Mix (Applied Biosystems, \#4444557) or TaqMan Universal Master Mix II (Applied Biosystems, \#4440040) for amplified cDNA. Samples were normalized to housekeeping genes: 18S ribosomal RNA (RN18S) and glyceraldehyde 3-phosphate dehydrogenase (Gapdh).

Taqman probes (Applied Biosystems): Lyz1, Mm00657323_m1; Chga, Mm00514341_m1, Gapdh, Mm99999915_g1; RN18S, Mm03928990_g1; Mki67, Mm01278617_m1;Cdkn1a (p21), Mm04205640_g1; Muc2, Mm01276696_m1; Ccnd1, Mm00432359_m1; Ror2, Mm01341765_m1/ Mm00443470_m1; Fltp, Mm01290543_g1; Fltp, Mm01290541_m1 Prickle1, Mm01297035_m1; Dvl2, Mm00432899_m1; Celsr1, Mm00464808_m1; Fzd6, Mm00433387_m1;Jun, Mm00495062_s1.

\section{Single-cell gene expression analysis by microfluidic qRT-PCR}

Small intestinal crypt cells were kept cold and sorted using FACS-Aria III (BD Bioscience). Doublets were excluded and dead cells were excluded using 7-AAD (eBioscience, \#00-699350). The pre-amplification solution in 96 -wells included $5 \mu 1$ of a master mix containing $1.2 \mu 1$ 5x VILO reaction mix (Invitrogen, \#11754-050), 0.3 $\mu 1$ 20U/ $\mu 1$ SUPERase-In (Ambion, \#AM2694), 0.25 $\mu 1$ 10\% NP40 (Thermo Scientific, \#28324), 0.25 $\mu 1$ RNA spikes mix (Fluidigm, 
\#100-5582) and $3 \mu 1$ nuclease-free water (Promega, \#P119C). Cells were lysed by incubation at $65^{\circ} \mathrm{C}$ for $90 \mathrm{~s}$ and RNA was transcribed into cDNA by adding $1 \mu 1$ of RT mix solution

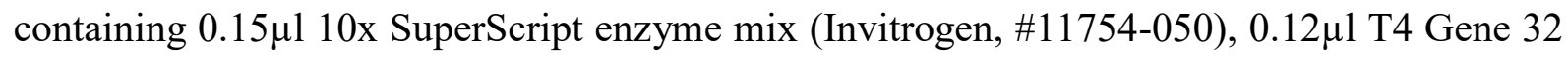
Protein (New England BioLabs, \#M0300S) and $0.73 \mu 1$ nuclease-free water and RT cycling $\left(25^{\circ} \mathrm{C}\right.$ for $5 \mathrm{~min}, 50^{\circ} \mathrm{C}$ for $30 \mathrm{~min}, 55^{\circ} \mathrm{C}$ for $25 \mathrm{~min}, 60^{\circ} \mathrm{C}$ for $5 \mathrm{~min}$ and $70^{\circ} \mathrm{C}$ for $10 \mathrm{~min}$ ). Target specific cDNA amplification was performed by adding $9 \mu 1$ reaction mix containing $7.5 \mu 1$ TaqMan PreAmp Master Mix (Applied Biosystems, \#4391128), 0.075 $\mu 1$ 0.5M EDTA, pH 8.0 (Invitrogen, \#Am9260G), 1.5 $\mu 1$ 10x outer primer mix (500nM) (see Supplementary Table 6) and 20 cycles of denaturation for $5 \mathrm{~s}$ at $96^{\circ} \mathrm{C}$ and 4 min annealing/extension at $60^{\circ} \mathrm{C}$ following an enzyme activation step at $95^{\circ} \mathrm{C}$ for $10 \mathrm{~min}$. Exonuclease I treatment was performed to clean up the reaction by adding a $6 \mu 1$ reaction mix containing $0.6 \mu 1$ reaction buffer, $1.2 \mu 1$ Exonuclease I (New England BioLabs, \#M0293S) and 4.2 $\mu 1$ nuclease-free water.

Amplified single-cell cDNAs were analysed with gene specific inner primer pairs (Supplementary Table 6) and SsoFast EvaGreen Supermix with Low ROX (Bio-Rad Laboratories, \#172-5210) using the $96 \times 96$ Dynamic Array on the BioMark System (Fluidigm). $\mathrm{Ct}$ values for each gene in each cell was calculated using BioMark Real-Time PCR Analysis software (Fluidigm).

\section{Computational analyses of single-cell qRT-PCR data}

Subsequent data processing and analysis of single cell qRT-PCR data was performed in R (http://www.r-project.org/). We manually removed 8 cells due to technical problems (pipetting error) during experimental processing. Samples were then corrected for deviating sample dilution of PCR runs. We followed the normalization procedure as suggested in9. Briefly, we subtracted $\mathrm{Ct}$ values from the assumed limit of detection of the BioMark ( $\mathrm{LOD}=30$ ). As quality control measure and reference we used the expression values of the three most robustly expressed housekeeping genes Rn18S, ActB and Hsp90. We excluded all cells that did not express all three housekeepers as well as cells for which the mean of the three housekeepers was \pm 3 s.d. from the mean of all cells. $\Delta \mathrm{Ct}$ values were then normalized on a cell-wise basis to the mean expression of the three housekeeping genes. The minimum of the normalized data - 2 was then assigned as a $\Delta \mathrm{Ct}$ value where a gene was not detected (failed qPCR runs). 595 of 672 sorted cells were retained for further analysis. Foxa1, Foxa2, GFP did not amplify correctly in one run so these genes have been excluded from the analysis as were all housekeeping genes (Rn18S, ActB, Hsp90, Uba52, Gapdh, Rpl37). We quantified three synthetic RNAs of different concentrations to explore its use as a reference. The signals of the RNA spikes were too strong 
and not quantifiable in all experimental runs and were therefore removed before further analyses. Fltp was detected in the negative control of Neurog3 cells and therefore had to be excluded from multivariate analyses. For the other cell types Fltp measurements were correct. In total, we used $\Delta \mathrm{Ct}$ values of 80 genes.

\section{Single-cell qPCR analysis}

All further analysis of single-cell qPCR data was performed using Scanpy (v.1.0.4). The singlecell neighborhood graph was computed on the 15 first principal components with a local neighborhood size of 5 (pp.pca and pp.neighbors) and UMAP was run for visualization (tl.umap). Louvain-based clustering at a resolution of 0.8 was used for subtype identification which were annotated based on the expression of known marker genes (pp.louvain). Genes characteristic for each subtype were identified using a wilcoxon-ranksum test (tl.rank_gene_groups). Top 5 ranked genes were considered for plotting. For the reconstruction of lineage relationships and differentiation trajectories we used PAGA (tl.paga) and diffusion pseudotime (dpt, tl.dpt). We first applied PAGA to find the branching into the EEC and Paneth cell lineage. For each lineage (branch in PAGA) we then arranged cells by their pseudotemporal order inferred from dpt (pl.paga_path). The root is represented by a cell in the ISC population, defined as $\mathrm{Lgr}^{+}$cells expressing the stem cell markers (Lgr5, Olfm4, Ascl2, Axin2 and Prom 1) but without specific lineage markers (Lyz1, Mmp 7, Atoh1, Dll4, Dll1 or Sis). Random variation of the root within the stem cell population did not substantially change dpt. Expression values along a trajectory are plotted as the smoothed average over $n$ cells using a sliding window with Gaussian noise as implemented in pl.paga_path ( $\mathrm{n}$ as indicated in figure legend).

\section{Single-cell RNA sequencing: RNA preparation, library generation and sequencing.}

Samples from SI crypts were prepared as described above under section crypt isolation and flow cytometry (Supplementary Table 2). For rare lineage enrichment live crypt cells were mixed with reporter positive cells at different ratios. Number of dead cells was estimated by trypan blue staining and sorted cells were counted. Single-cell libraries were generated using the Chromium ${ }^{\mathrm{TM}}$ Single cell 3' library and gel bead kit v2 (10X Genomics, \#120237) according to the manufacturer's instructions. Briefly, cells were loaded onto a channel of the 10X chip to generate Gel Bead-in-Emulsions (GEMs). These underwent reverse transcription to barcode RNA before cleanup and cDNA amplification. Afterwards, enzymatic fragmentation and attachment of $5^{\prime}$ adaptor and sample index was performed. Libraries were sequenced on the HiSeq4000 (Illumina) with $150 \mathrm{bp}$ paired-end sequencing of read 2. 


\section{Computational analysis}

\section{Pre-processing of droplet-based scRNAseq data}

De-multiplexing and alignment to mm10 mouse genome, identification of unique molecular identifiers (UMI) and barcode filtering was performed using the 'CellRanger' toolkit (version 2.0.0) provided by $10 \mathrm{X}$ Genomics. We performed a further barcode (=cell) selection step and additionally included cells with more than 1000 expressed genes, where a gene is counted as expressed if we found at least one UMI mapped to it. We further filtered cells with a fraction of counts from mitochondrial genes $>10 \%$ indicative for stressed or dying cells.

We removed doublets by computing the doublet score from scrublet ${ }^{42}$ on the UMI count matrix separately for every sample. Using a threshold of 0.4 , we removed 1651 cells from the analysis.

Cells from all samples were log-normalised and batch corrected using ComBat as Python implementation. Please note that we observed an overrepresentation of Paneth cells in the FVRenriched samples (with 50\% and 90\% enrichment, resp.), where we subsampled the Paneth cell populations to $15 \%$ to fit the cell-type distribution of all other samples before we corrected with ComBat. Then, we used the pre-computed regression coefficients to correct for batch effects in the filtered cells and merged them with the full data set. Further, we fixed all zero values to remain zero in order to preserve the support of the count data. We computed the top 2000 highly variable genes based on mean and dispersion (pp.filter_genes_dispersion in SCANPY v. 1.3.1 in Python 3.6 with the flavor 'cell_ranger' to compute normalised dispersions ${ }^{43}$ ).

Further, we corrected for the library size by scaling the reads per cell to the factor 100,000 (pp.normalize_per_cell in SCANPY v. 1.3.1 in Python 3.6).

\section{Dimension reduction}

We performed our analyses with $\mathrm{SCANPY}^{44}$ v. 1.3.1 in Python 3.6. We used a UMAP ${ }^{45}$ to represent the data in the two-dimensional embedding and data visualisation (tl.umap). This was created based on a PCA-space with $\mathrm{n}=50$ components, and the k-nearest neighbour graph on the PCA-space with $\mathrm{k}=30$ (tl.pca and pp.neighbors). PCA etc. was computed on the scaled and normalised data with 2,000 highly variable genes.

\section{Clustering and cell type annotation}

We determined the clustering and cell type annotation for the control samples as follows. We inspected first marker gene expression for respective major cell types and computed gene scores 
using known marker genes (tl.score_genes, based on ref. ${ }^{46}$ ). Analogously, we computed a cell cycle score to determine the respective cell-cycle phase state of the cells (tl.score_genes_cell_cycle, based on ref. ${ }^{46}$ ). Then, we performed clustering using the louvain algorithm $^{47}$ (tl.louvain with default resolution parameter 1.0) and found 18 clusters. Here, we annotated and merged clusters again according to the gene scores and marker gene expression. Here, we also identified 1589 immune cells, which were distinct from the remaining cells.

Subsequently, we inspected all main clusters for substructure and resolved it further based on marker gene expression. Here, we used louvain clustering with resolution parameters $\sim 1-2$ and merged the subclusters again according to marker gene expression (hierarchical 'split-andmerge' approach). Finally, we annotated 7 major cell types (ISC, Enterocytes, Goblet cells, Paneth cells, Enteroendocrine cells and Tuft cells), subdivided them into progenitor and mature cells. In the ISC population, we identified a PC-primed ISC population that was more similar to the Paneth progenitor population.

For the mutant samples, we employed a k-nearest neighbour approach to match every cell to the corresponding cluster $(\mathrm{k}=30)$, i.e. we derived the cell identity of the mutants from the cell identity of neighbouring cells of the control samples, which we annotated beforehand. Again, we identified 2289 immune cells, which we removed from analysis.

\section{Differential expression analysis}

We used the limma package ${ }^{48}$ (version 3.34.9 in R 3.4.3) to study differential expression. First, we excluded the FVF enriched samples and mutant samples. In order to determine differentially expressed genes between ISCs and progenitor populations, we tested pairwise progenitor populations vs ISC (without Paneth primed ISCs) and Paneth progenitors vs Goblet progenitors. Please note that differential expression was performed on the log-normalised (not batchcorrected) data, where we included the sample information (i.e. batch) as covariate. In addition, we excluded all genes with mean expression $<0.05$. We considered only significant genes $(\mathrm{FDR}<0.05)$ with $\log \mathrm{FC}>0.05$. For the analysis of transcription factors, we filtered differentially expressed transcription factors (gene ontology ID GO:0003700 (transcription factor activity)) with the biomartr package ${ }^{49}$ (version 0.7.0 in R 3.4.3) with a p-value threshold $p_{\text {adj }}<10^{-5}$.

Analogously, in order to determine differentially expressed genes between mutants and control samples (without FVF enriched samples), we tested every cluster separately with limma. Please note that differential expression was performed on the log-normalised (not batch-corrected) data, where we included the number of expressed genes as covariate, but not the sample information (i.e. batch) due to confounding of genetic condition and sample covariates. In 
addition, we excluded all genes with mean expression $<0.05$. We considered only significant genes $(\mathrm{FDR}<0.05)$ with $\log \mathrm{FC}>0.05$. For the analysis of transcription factors, we filtered differentially expressed transcription factors (gene ontology ID GO:0003700 (transcription factor activity)) with the biomartr package (version 0.7.0 in R 3.4.3) and with an adjusted pvalue threshold $\mathrm{p}_{\mathrm{adj}}<10^{-5}$ (Benjamini-Hochberg correction).

\section{Identifying cell differentiation trajectories via graph abstraction}

To derive cell trajectories, we computed a pseudotemporal ordering using diffusion pseudotime (DPT, tl.dpt in SCANPY $)^{50}$. As the topology of the data is complex, we used partition-based graph abstraction (PAGA) ${ }^{33}$ to quantify the connections between the clusters (i.e. connections of ISCs to respective progenitors and mature cell types, tl.paga in SCANPY). We display all connections with a scaled connectivity of at least 0.05 ('threshold' parameter in pl.paga in SCANPY).

\section{Gene Set Enrichment Analysis}

We performed a gene set enrichment analysis based on both $\mathrm{GO}^{51,52}$ terms and $\mathrm{KEGG}^{53}$ terms using g:profiler ${ }^{54}$. In particular, we adapted the Python wrapper from V. Svensson (https://github.com/vals/python-gprofiler). We set the background to all expressed genes and removed all resulting gene sets with significance level $p>=0.05$. Further, we split the input data set by the sign of the log-fold change, such that we considered up-regulated and down-regulated gene sets separately in each set of differentially expressed genes. For visualisation of gene set significance, we abridged p-values at $10^{-10}$.

\section{Code and data accession.}

Custom R scripts of the single cell qRT-PCR bioinformatics analyses are available in a jupyter notebook upon request.

Microarray data have been submitted to NCBI/GEO (GSE94092).

scRNAseq data have been submitted to NCBI/GEO (code upon request). 


\section{Statistical analysis}

No statistical methods were used to predetermine sample size. The experiments were not randomized and the investigators were not blinded to allocation during experiments and outcome assessment.

Statistical analysis was performed using GraphPad Prism 6 Software (GraphPad Software, USA). Data are expressed as values and were compared using unpaired t-tests or ANOVA, unless indicated otherwise. 


\section{Supplementary Table 1}

Shows the differentially expressed genes in the FVR populations.

\section{Supplementary Table 2}

Overview of samples used for scRNAseq analysis.

\section{Supplementary Table 3}

Shows the enrichment of rare lineages with Fltp ${ }^{\mathrm{ZV}}$ and FVF reporter mice.

\section{Supplementary Table 4}

Shows the genes used to determine the gene scores.

\section{Supplementary Table 5}

Genes used in single-cell qRT-PCR analysis.

\section{Supplementary Table 6}

Primer for single-cell qRT-PCR. 


\section{References}

1. Barker, N. et al. Identification of stem cells in small intestine and colon by marker gene Lgr5. Nature (2007) doi:10.1038/nature06196.

2. Grün, D. et al. De Novo Prediction of Stem Cell Identity using Single-Cell Transcriptome Data. Cell Stem Cell (2016) doi:10.1016/j.stem.2016.05.010.

3. Li, N., Nakauka-Ddamba, A., Tobias, J., Jensen, S. T. \& Lengner, C. J. Mouse LabelRetaining Cells Are Molecularly and Functionally Distinct From Reserve Intestinal Stem Cells. Gastroenterology (2016) doi:10.1053/j.gastro.2016.04.049.

4. Li, N. et al. Single-cell analysis of proxy reporter allele-marked epithelial cells establishes intestinal stem cell hierarchy. Stem Cell Reports (2014) doi:10.1016/j.stemcr.2014.09.011.

5. Kim, T. H. et al. Single-Cell Transcript Profiles Reveal Multilineage Priming in Early Progenitors Derived from Lgr5+ Intestinal Stem Cells. Cell Rep. (2016) doi:10.1016/j.celrep.2016.07.056.

6. Potten, C. S. Stem cells in gastrointestinal epithelium: Numbers, characteristics and death. Philos. Trans. R. Soc. B Biol. Sci. (1998) doi:10.1098/rstb.1998.0246.

7. Buczacki, S. J. A. et al. Intestinal label-retaining cells are secretory precursors expressing Igr5. Nature (2013) doi:10.1038/nature11965.

8. Grün, D. et al. Single-cell messenger RNA sequencing reveals rare intestinal cell types. Nature (2015) doi:10.1038/nature14966.

9. Heuberger, J. et al. Shp2/MAPK signaling controls goblet/paneth cell fate decisions in the intestine. Proc. Natl. Acad. Sci. U. S. A. (2014) doi:10.1073/pnas.1309342111.

10. Shroyer, N. F., Wallis, D., Venken, K. J. T., Bellen, H. J. \& Zoghbi, H. Y. Gfi1 functions downstream of Math1 to control intestinal secretory cell subtype allocation and differentiation. Genes Dev. (2005) doi:10.1101/gad.1353905.

11. van Es, J. H. et al. Dll1 marks early secretory progenitors in gut crypts that can revert to stem cells upon tissue damage. Nat. Cell Biol. (2012) doi:10.1038/ncb2581.Dll1.

12. Schonhoff, S. E., Giel-Moloney, M. \& Leiter, A. B. Neurogenin 3-expressing progenitor cells in the gastrointestinal tract differentiate into both endocrine and non-endocrine cell types. Dev. Biol. (2004) doi:10.1016/j.ydbio.2004.03.013.

13. Gehart, H. \& Clevers, H. Tales from the crypt: new insights into intestinal stem cells. Nature Reviews Gastroenterology and Hepatology (2019) doi:10.1038/s41575-0180081-y.

14. Fre, S. et al. Notch signals control the fate of immature progenitor cells in the intestine. Nature 435, 964-968 (2005).

15. Yang, Q., Bermingham, N. A., Finegold, M. J. \& Zoghbi, H. Y. Requirement of Math1 for secretory cell lineage commitment in the mouse intestine. Science (80-. ). (2001) doi:10.1126/science.1065718. 
16. Ritsma, L. et al. Intestinal crypt homeostasis revealed at single-stem-cell level by in vivo live imaging. Nature (2014) doi:10.1038/nature12972.

17. Takeda, N. et al. Interconversion between intestinal stem cell populations in distinct niches. Science (80-. ). (2011) doi:10.1126/science.1213214.

18. Sangiorgi, E. \& Capecchi, M. R. Bmi1 is expressed in vivo in intestinal stem cells. Nat. Genet. (2008) doi:10.1038/ng.165.

19. Montgomery, R. K. et al. Mouse telomerase reverse transcriptase (mTert) expression marks slowly cycling intestinal stem cells. Proc. Natl. Acad. Sci. U. S. A. (2011) doi:10.1073/pnas.1013004108.

20. Powell, A. E. et al. The pan-ErbB negative regulator Irig1 is an intestinal stem cell marker that functions as a tumor suppressor. Cell (2012) doi:10.1016/j.cell.2012.02.042.

21. Kozar, S. et al. Continuous clonal labeling reveals small numbers of functional stem cells in intestinal crypts and adenomas. Cell Stem Cell (2013) doi:10.1016/j.stem.2013.08.001.

22. Cortijo, C., Gouzi, M., Tissir, F. \& Grapin-Botton, A. Planar Cell Polarity Controls Pancreatic Beta Cell Differentiation and Glucose Homeostasis. Cell Rep. (2012) doi:10.1016/j.celrep.2012.10.016.

23. Bader, E. et al. Identification of proliferative and mature beta-cells in the islets of Langerhans. Nature 535, 430-+ (2016).

24. Roscioni, S. S., Migliorini, A., Gegg, M. \& Lickert, H. Impact of islet architecture on $\beta$ cell heterogeneity, plasticity and function. Nature Reviews Endocrinology (2016) doi:10.1038/nrendo.2016.147.

25. Grumolato, L. et al. Canonical and noncanonical Wnts use a common mechanism to activate completely unrelated coreceptors. Genes Dev. (2010) doi:10.1101/gad.1957710.

26. Niehrs, C. The complex world of WNT receptor signalling. Nature Reviews Molecular Cell Biology (2012) doi:10.1038/nrm3470.

27. Gegg, M. et al. Flattop regulates basal body docking and positioning in mono- and multiciliated cells. Elife 3, (2014).

28. Muñoz, J. et al. The Lgr5 intestinal stem cell signature: Robust expression of proposed quiescent ' +4' cell markers. EMBO J. (2012) doi:10.1038/emboj.2012.166.

29. Haber, A. L. et al. A single-cell survey of the small intestinal epithelium. Nature (2017) doi:10.1038/nature24489.

30. Lange, A. et al. FltpT2AiCre: A new knock-in mouse line for conditional gene targeting in distinct mono- and multiciliated tissues. Differentiation 83, S105-S113 (2012).

31. Muzumdar, M. D., Tasic, B., Miyamichi, K., Li, N. \& Luo, L. A global double-fluorescent cre reporter mouse. Genesis (2007) doi:10.1002/dvg.20335. 
32. Burtscher, I. \& Lickert, H. Foxa2 regulates polarity and epithelialization in the endoderm germ layer of the mouse embryo. Development (2009) doi:10.1242/dev.028415.

33. Wolf, F. A. et al. PAGA: graph abstraction reconciles clustering with trajectory inference through a topology preserving map of single cells. Genome Biol. (2019) doi:10.1186/s13059-019-1663-x.

34. Kim, T. H. et al. Broadly permissive intestinal chromatin underlies lateral inhibition and cell plasticity. Nature (2014) doi:10.1038/nature12903.

35. Weinreb, C., Rodriguez-Fraticelli, A., Camargo, F. D. \& Klein, A. M. Lineage tracing on transcriptional landscapes links state to fate during differentiation. Science (80-. ). (2020) doi:10.1126/science.aaw3381.

36. Wagner, D. E. \& Klein, A. M. Lineage tracing meets single-cell omics: opportunities and challenges. Nature Reviews Genetics (2020) doi:10.1038/s41576-020-0223-2.

37. Bastidas-Ponce, A. et al. Comprehensive single cell mRNA profiling reveals a detailed roadmap for pancreatic endocrinogenesis. Dev. (2019) doi:10.1242/dev.173849.

38. Curtin, J. A. et al. Mutation of Celsr1 disrupts planar polarity of inner ear hair cells and causes severe neural tube defects in the mouse. Curr. Biol. (2003) doi:10.1016/S09609822(03)00374-9.

39. Burtscher, I., Barkey, W. \& Lickert, H. Foxa2-venus fusion reporter mouse line allows live-cell analysis of endoderm-derived organ formation. Genesis (2013) doi:10.1002/dvg.22404.

40. Andersson-Rolf, A., Fink, J., Mustata, R. C. \& Koo, B. K. A video protocol of retroviral infection in primary intestinal Organoid culture. J. Vis. Exp. (2014) doi:10.3791/51765.

41. Yin, X. et al. Niche-independent high-purity cultures of Lgr5 + intestinal stem cells and their progeny. Nat. Methods (2014) doi:10.1038/nmeth.2737.

42. Wolock, S. L., Lopez, R. \& Klein, A. M. Scrublet: Computational Identification of Cell Doublets in Single-Cell Transcriptomic Data. Cell Syst. (2019) doi:10.1016/j.cels.2018.11.005.

43. Zheng, G. X. Y. et al. Massively parallel digital transcriptional profiling of single cells. Nat. Commun. (2017) doi:10.1038/ncomms14049.

44. Wolf, F. A., Angerer, P. \& Theis, F. J. SCANPY: Large-scale single-cell gene expression data analysis. Genome Biol. (2018) doi:10.1186/s13059-017-1382-0.

45. McInnes, L., Healy, J., Saul, N. \& Großberger, L. UMAP: Uniform Manifold Approximation and Projection. J. Open Source Softw. (2018) doi:10.21105/joss.00861.

46. Satija, R., Farrell, J. A., Gennert, D., Schier, A. F. \& Regev, A. Spatial reconstruction of single-cell gene expression data. Nat. Biotechnol. (2015) doi:10.1038/nbt.3192.

47. Blondel, V. D., Guillaume, J. L., Lambiotte, R. \& Lefebvre, E. Fast unfolding of communities in large networks. J. Stat. Mech. Theory Exp. (2008) doi:10.1088/1742- 
$5468 / 2008 / 10 / P 10008$

48. Ritchie, M. E. et al. Limma powers differential expression analyses for RNA-sequencing and microarray studies. Nucleic Acids Res. (2015) doi:10.1093/nar/gkv007.

49. Drost, H. G. \& Paszkowski, J. Biomartr: Genomic data retrieval with R. Bioinformatics (2017) doi:10.1093/bioinformatics/btw821.

50. Haghverdi, L., Buttner, M., Wolf, F. A., Buettner, F. \& Theis, F. J. Diffusion pseudotime robustly reconstructs lineage branching. Nat. Methods 13, 845-+ (2016).

51. Ashburner, M. et al. Gene ontology: Tool for the unification of biology. Nature Genetics (2000) doi:10.1038/75556.

52. Carbon, S. et al. Expansion of the gene ontology knowledgebase and resources: The gene ontology consortium. Nucleic Acids Res. (2017) doi:10.1093/nar/gkw1108.

53. Kanehisa, M., Sato, Y., Kawashima, M., Furumichi, M. \& Tanabe, M. KEGG as a reference resource for gene and protein annotation. Nucleic Acids Res. (2016) doi:10.1093/nar/gkv1070.

54. Reimand, J. et al. g:Profiler-a web server for functional interpretation of gene lists (2016 update). Nucleic Acids Res. (2016) doi:10.1093/nar/gkw199. 
Acknowledgements We thank Kerstin Diemer, Jürgen Schultheiss, Ines Kunze, Anita Ludwig, and Anke Bettenbrock for excellent technical assistance, and Aurelia Raducanu and Pallavi Mahaddalkar for assistance with the FACS, and Henner Farin for teaching intestinal single cell culture. We are grateful to Jennifer Murdoch for providing Celsr $1^{\text {crsh }}$ animals and Helena Edlund for providing the Ngn3 antibody. We thank Ralph Böttcher, Matthias Tschoep and Steve Woods for critical reading the manuscript. This work was supported by an EmmyNoether Fellowship and the European Union with the ERC starting grant Ciliary Disease and HumEn project. This work was funded by the Helmholtz Alliance ICEMED - Imaging and Curing Environmental Metabolic Diseases (H.L., J.B.) and through the Initiative and Networking Fund of the Helmholtz Association (H.L.). For financial support we would also like to thank the Helmholtz Society, Helmholtz Portfolio Theme 'Metabolic Dysfunction and Common Disease' (H.L. and J.B.), the Helmholtz Alliance 'Aging and Metabolic Programming, AMPro' (H.L., J.B.). Further, this projected was funded by ExNet-0041-Phase2-3 (,SyNergyHMGU“) through the Initiative and354Network Fund of the Helmholtz Association (H.L., F.J.T.), the German Research Foundation and the German Center for Diabetes Research (DZD e.V.) (H.L.).

Authors Contributions A.B. designed experiments, performed experiments, analysed data and wrote the manuscript. M.B. analysed single-cell qRT-PCR and RNAseq data. S.T. analysed single-cell qPCR data. M.S. analysed microarray data. A.A. performed western blot analysis of sorted cell populations. I.B. generated Ngn3-VF mouse line. S.S. analysed microarray data. M.I. and J.B. performed the microarrays and data analysis. C.Z. and W.E. contributed to single cell qPCR experiment and discussions. A.C.S., F.M.V. and O.E. provided single-cell qRT-PCR resources and contributed to discussions. F.J.T. supervised M.B., S.T. and S.S. and analysed single-cell RNAseq and qRT-PCR data. H.L. helped in study design, analysis and writing and acquired financial support.

\section{Competing interests}

F.J.T. reports receiving consulting fees from Roche Diagnostics GmbH and Cellarity Inc., and ownership interest in Cellarity, Inc. and Dermagnostix. S.T. reports receiving consulting fees from Cellarity, Inc. All other authors declare no conflict of interest. 


\section{Böttcher et al. Figure 1}

FVR labels the secretory enteroendocrine and Paneth cell lineages.

a

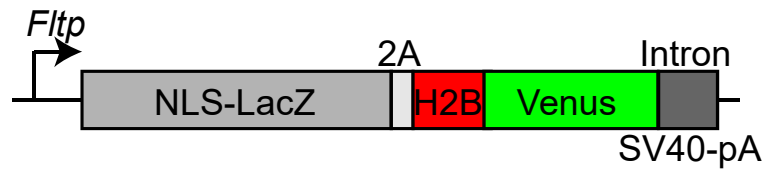

b

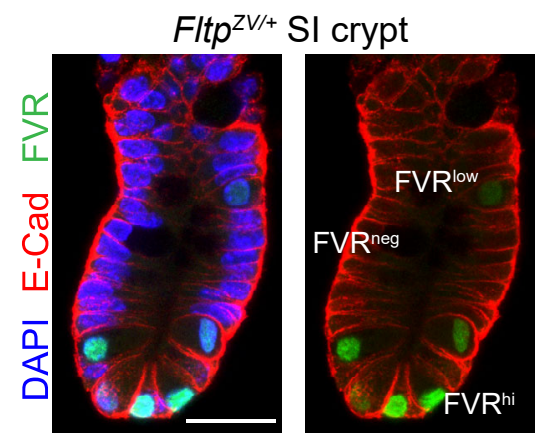

d

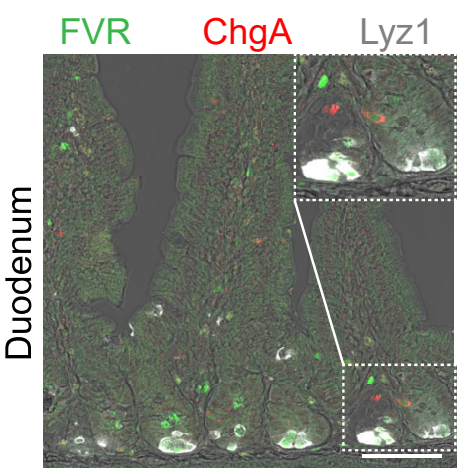

DAPI

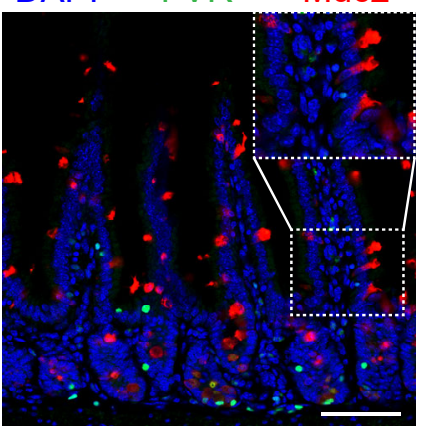

g

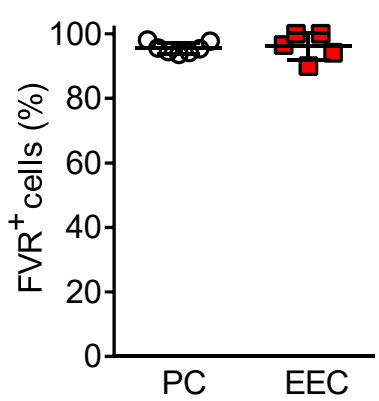

f

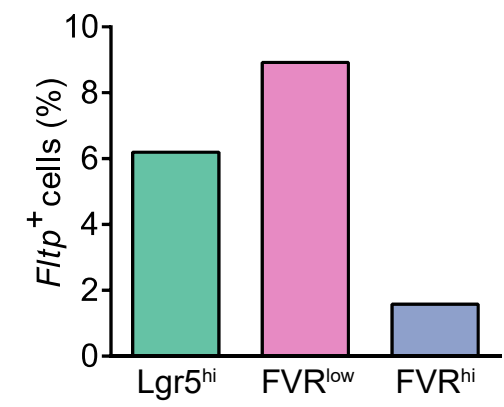

C

SI crypt isolation Fltp ${ }^{2 V /+}$
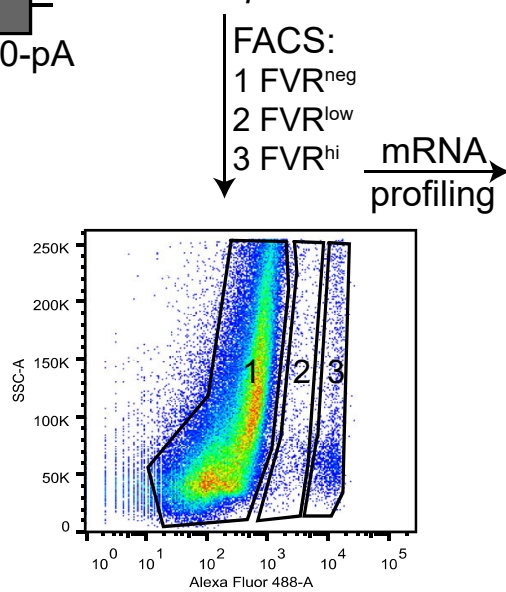

\begin{tabular}{lll}
1.5 & 0 & -1.5 \\
\hline & &
\end{tabular}

$F^{\text {neg }} \quad F V R^{\text {low }} \quad F V R^{\text {hi }}$

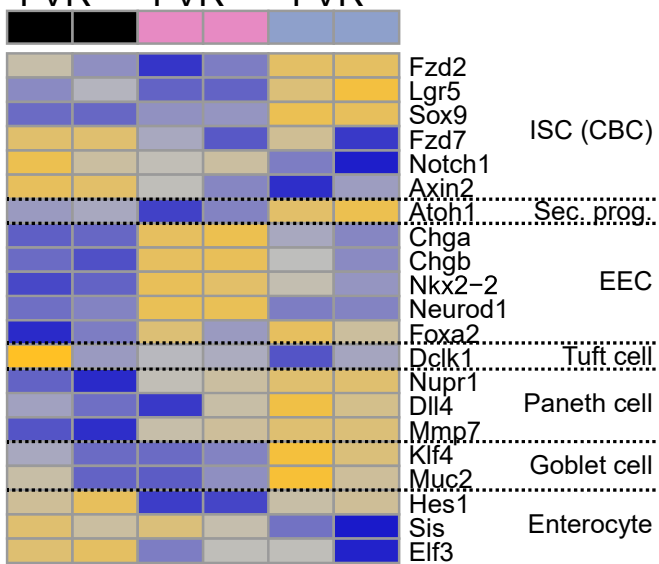

DAPI FVR Dclk1

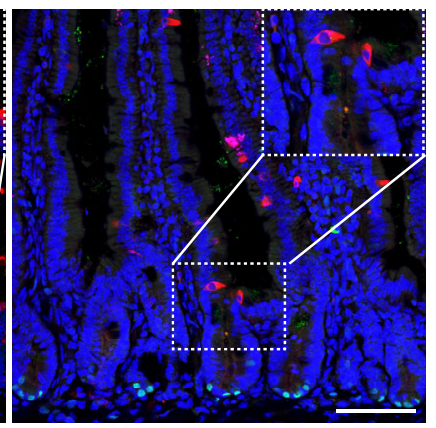

Fltp $^{z V /+}$ SI crypt

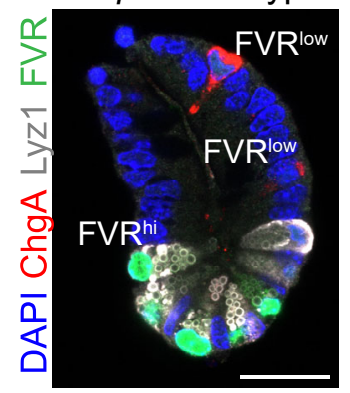

h

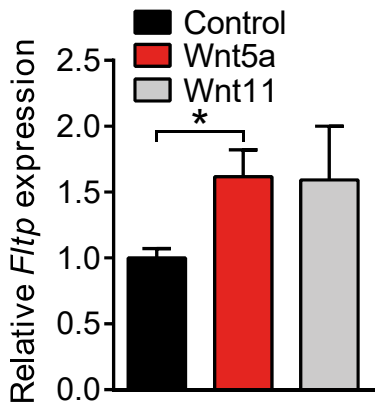


Figure 1 FVR labels the secretory enteroendocrine and Paneth cell lineages.

a, Schematic of the loss-of-function and NLS-LacZ/H2B-Venus transcriptional reporter allele $\left(\right.$ Fltp $\left.^{Z V}\right)$ for Fltp.

b, Laser scanning confocal microscopy (LSM) image of a representative small intestinal (SI) crypt isolated from a Fltp ${ }^{Z V /+}$ reporter mouse line depicting FVR ${ }^{\text {hi/low/neg }}$ crypt cells. FVR (Venus, green), DAPI (blue, nuclei), E-cad (red, membrane). Scale bar, $25 \mu \mathrm{m}$.

c, Experimental design for microarray analysis of the three small intestinal (SI) crypt cell populations distinguishable by FVR activity. The heatmap depicts the expression profiles of key stem-cell and intestinal lineage genes. Expression is scaled row-wise and the colours range from dark blue (low expression) to orange (high expression) and represent normalized expression (row z-score). ISC, intestinal stem cell. CBC, crypt base columnar cell. Sec. progenitor, secretory progenitor. EEC, enteroendocrine cell.

d, LSM images showing FVR (Venus, green) expression in the secretory lineages in the adult intestine of Fltp ${ }^{Z V /+}$ mice (4 months) co-stained against ChgA (red, enteroendocrine cells), Lyz1 (white, Paneth cells), Muc2 (red, goblet cells), and Dclk1 (red, tuft cells). DAPI (blue) stains the nucleus. Scale bars, $75 \mu \mathrm{m}$.

e, f, LSM image depicting a representative SI crypt isolated from adult Fltp ${ }^{Z V /+}$ reporter mice with indicated FVR ${ }^{\text {neg/low/hi }}$ cells stained for DAPI (blue, nucleus), FVR (Venus, green), ChgA (red, enteroendocrine cells), and Lyz1 (white, Paneth cells) (e) and quantification of $\mathrm{Lyz}^{+} \mathrm{FVR}^{+}$Paneth cells (PCs) and $\mathrm{ChgA}^{+} \mathrm{FVR}^{+}$enteroendocrine cells (EECs) (f). For Paneth cells: $n$ (mice) $=8$ with 99 analysed crypts $\left(95.58 \%\right.$ of $\left.\mathrm{PCs}=\mathrm{FVR}^{+}\right)$. For enteroendocrine cells: $n$ (mice) $=5$ with 76 analysed crypts $\left(96.13 \%\right.$ of EECs $\left.=\mathrm{FVR}^{+}\right)$. Scale bar, $25 \mu \mathrm{m}$.

g, Relative abundance of Fltp ${ }^{+} \mathrm{Lgr}^{\mathrm{hi}}, \mathrm{FVR}^{\text {low }}$ and FVR ${ }^{\text {hi }}$ cells determined by single-cell qRTPCR. $n=145 \mathrm{Lgr}^{\text {hi }}$ cells; $n=112 \mathrm{FVR}^{\text {low }}$ cells; $n=126 \mathrm{FVR}^{\text {hi }}$ cells. $n$ (mice) $=3$ for Fltp ${ }^{\mathrm{ZV} /+}$, Lgr5-ki

h, Fltp expression in crypts treated with indicated Wnt/PCP ligands for two days. $n$ (independent experiments $)=4$. Error bars represent SEM. Two-tailed Student's $t$-test, $* P<0.05, * * * P<0.001$. 


\section{Böttcher et al. Figure 2}

Wnt/ $\beta$-catenin and Wnt/PCP activated Lgr5+ ISCs are indistinguishable at the transcriptional level.

Fltp ${ }^{\text {T2AiCre/t+ }} ; \operatorname{Gt}(R O S A) 26^{m T m G /+}$

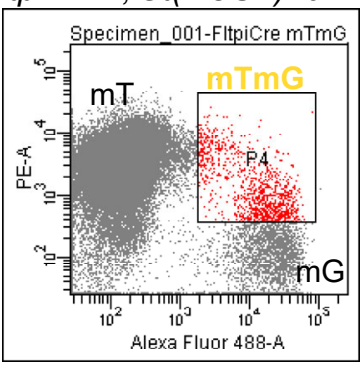

b

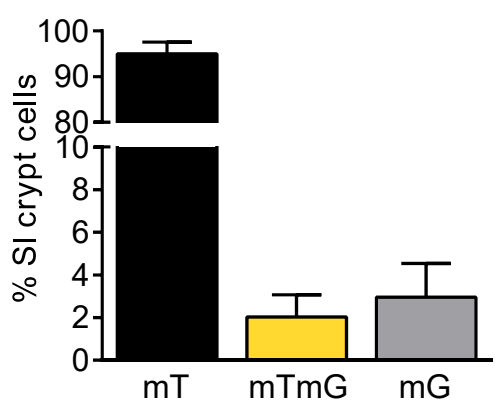

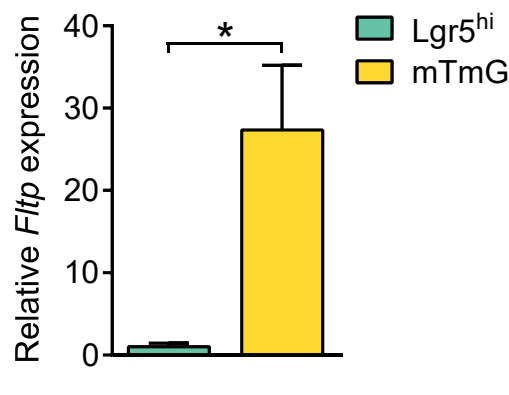

d

Fltp ${ }^{\text {T2AiCre/+ }} ;$ Gt(ROSA)26 $6^{m T m G /+}$

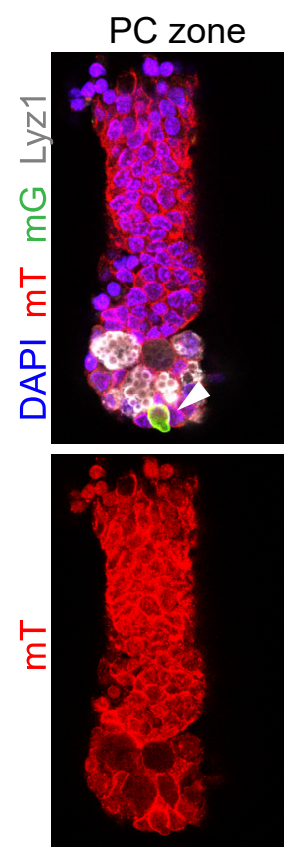

g
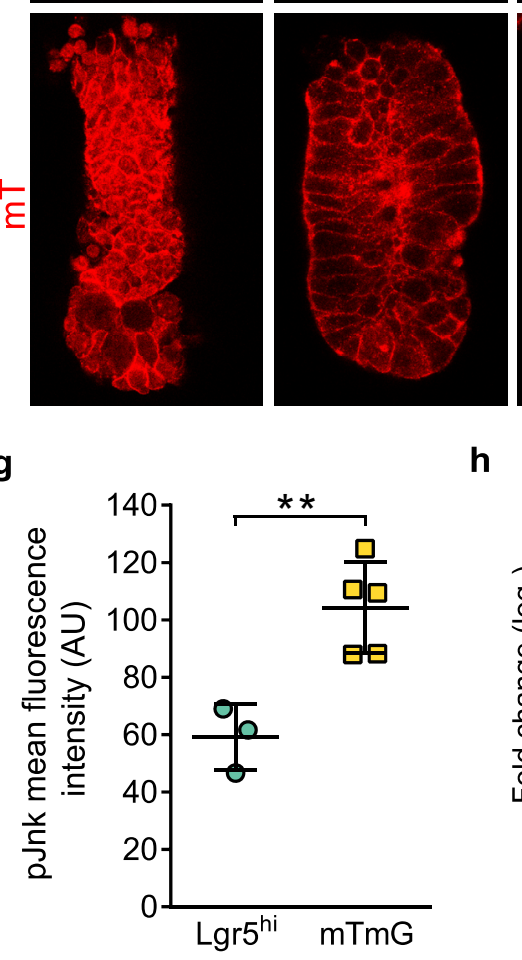

h e

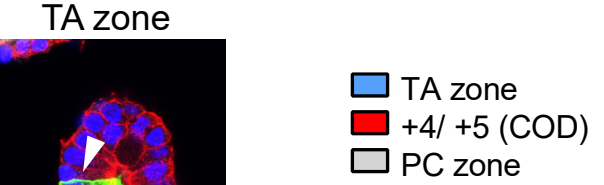

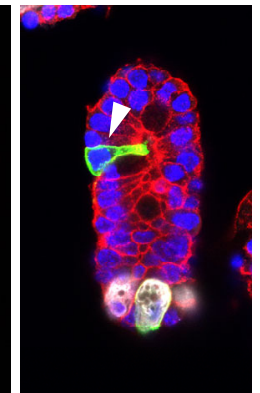
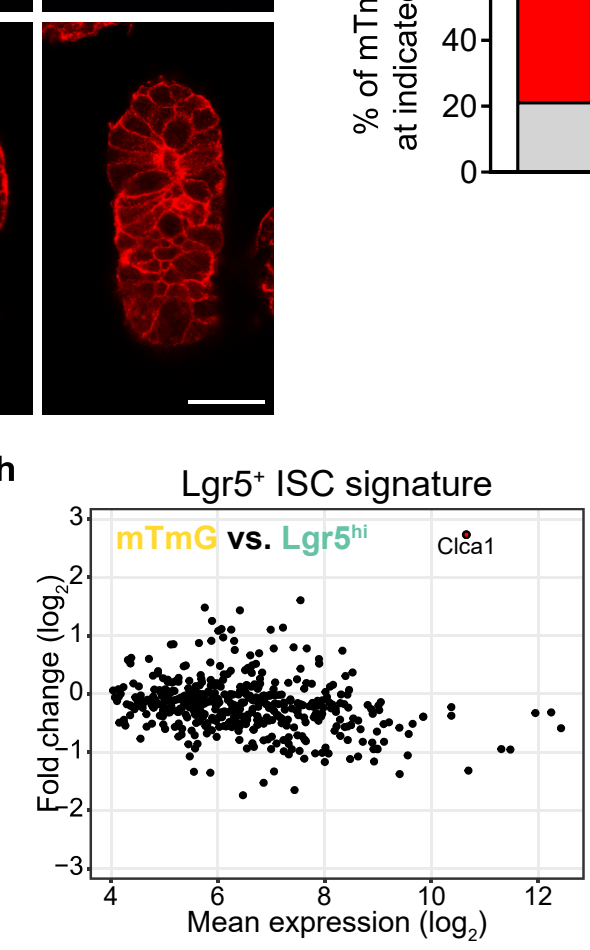

-Differentially expressed genes (FDR < 0.01)

- Similarily expressed genes (FDR >0.01) f

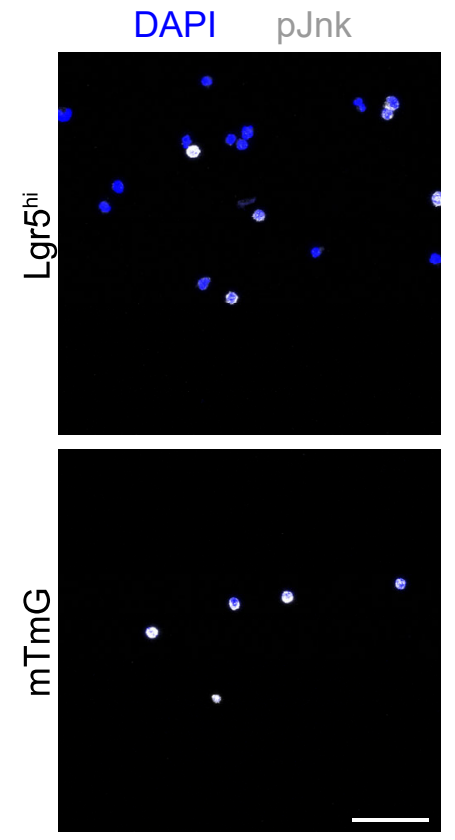

i

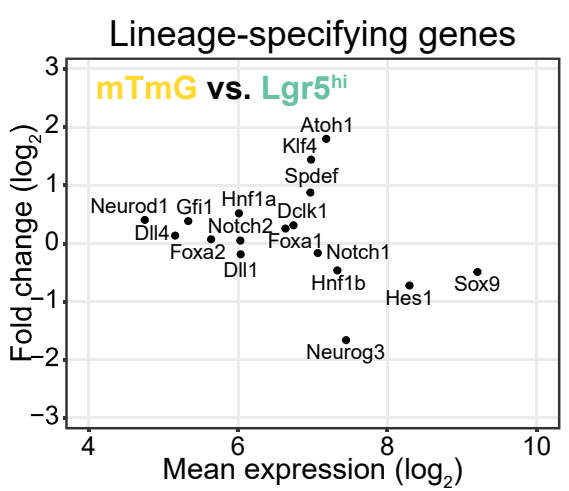

- Differentially expressed genes (FDR $<0.01)$

-Similarily expressed genes (FDR $>0.01)$ 
Figure $2 \mathrm{Wnt} / \beta$-catenin and Wnt/PCP activated $\mathrm{Lgr}^{+}$ISCs are indistinguishable at the transcriptional level.

a, b, Flow cytometry analysis (a) and relative abundance (b) of Fltp lineage $(\mathrm{mT})$, Fltp ${ }^{+}$ intermediate cells $(\mathrm{mTmG})$ and Fltp lineage ${ }^{+}(\mathrm{mG})$ crypt cells isolated from Fltp ${ }^{T 2 A i C r e /+} ; G t(\operatorname{ROSA}) 26^{m T m G /+}$ mice. $n($ mice $)=6$. Error bars represent SD.

c, qRT-PCR data comparing relative Fltp expression in $\mathrm{mTmG}$ crypt cells and Lgr $^{\text {hi }}$ intestinal stem cells. $n$ (independent experiments) $=3$ for Lgr5 $5^{\text {hi }}$ cells. $n$ (independent experiments) $=2(4$ mice) for $\mathrm{mTmG}$ cells. Error bars represent SD. Two-tailed Students's $t$-test, ${ }^{*} P<0.05$.

d, e, Representative LSM images of Fltp ${ }^{+}$intermediate $\mathrm{mTmG}$ cells at indicated positions. SI crypts from $\mathrm{Fltp}^{\mathrm{T2AiCre/+}} ; \mathrm{Gt}(\mathrm{ROSA}) 26^{m T m G /+}$ mice were stained for DAPI (blue, nucleus), mT (Fltp lineage ${ }^{-}$, RFP, green), $\mathrm{mG}$ (Fltp ${ }^{+}$intermediate cells, green, GFP), and Lyz1 (white, Paneth cells). Scale bar, $25 \mu \mathrm{m}$. (d). Abundance of $\mathrm{mTmG}$ cells at indicated positions $(\mathbf{e}) . n$ (mice) $=3$.

f, $\mathbf{g}$, Representative LSM images of $\mathrm{Lgr} 5^{\text {hi }}$ and $\mathrm{mTmG}$ cells isolated by flow cytometry and stained for active, phosphorylated Jun N-terminal kinase (pJnk, white) indicating active Wnt/PCP signalling and DAPI (blue, stains nuclei) (f). Quantification of the mean fluorescent intensity of pJnk (g). $n$ (independent experiments) $=3$ for Lgr $5^{\text {hi }}$ cells. $n$ (independent experiments) $=5$ for $\mathrm{mTmG}$ cells. Error bars represent SD. Two-tailed Student's $t$-test, ${ }^{*} P<0.05$. Scale bar, $50 \mu \mathrm{m}$.

h, MA-plot comparing the expression of ISC signature genes in $\mathrm{mTmG}$ and $\mathrm{Lgr}^{\text {hi }}$ (ISCs) cells. Differentially expressed genes (FDR $<0.01)$ are indicated in red. The y-axis indicates the fold change in $\log 2$ and the $\mathrm{x}$-axis indicates the mean $\log 2$ expression value. Clcal is the only significantly regulated gene. $n$ (Lgr5 ${ }^{\text {hi }}$ ISC microarray samples) $=6 . n$ (mTmG microarray samples) $=4$.

i, MA-plot comparing the expression of lineage-specifying genes in $\mathrm{mTmG}$ and Lgr $5^{\text {hi }}$ cells. The $\mathrm{y}$-axis indicates the fold change in $\log 2$ and the $\mathrm{x}$-axis indicates the mean $\log 2$ expression value. No gene is significantly regulated. $n$ (microarray samples) $=6$ for $\operatorname{Lgr} 5^{\text {hi }}$ ISC. $n$ (microarray samples) $=4$ for $\mathrm{mTmG}$. 


\section{Böttcher et al. Figure 3}

a

Subtype enrichment of crypt cells reveals distinct progenitor states for all intestinal lineages.

SI $\quad$-/+ Rare lineage enrichment
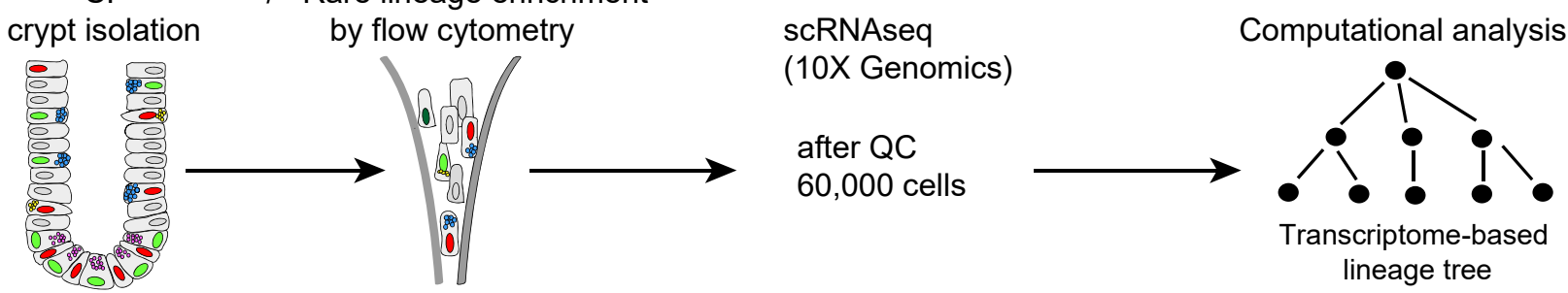

b

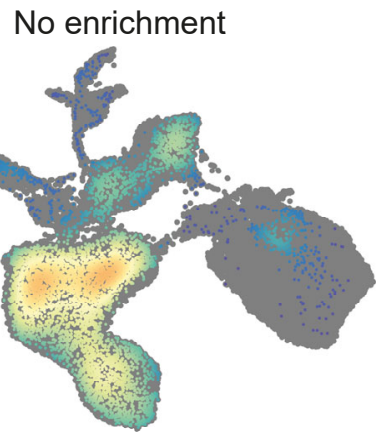

FVR enrichment (50\%)

FVR enrichment (90\%)
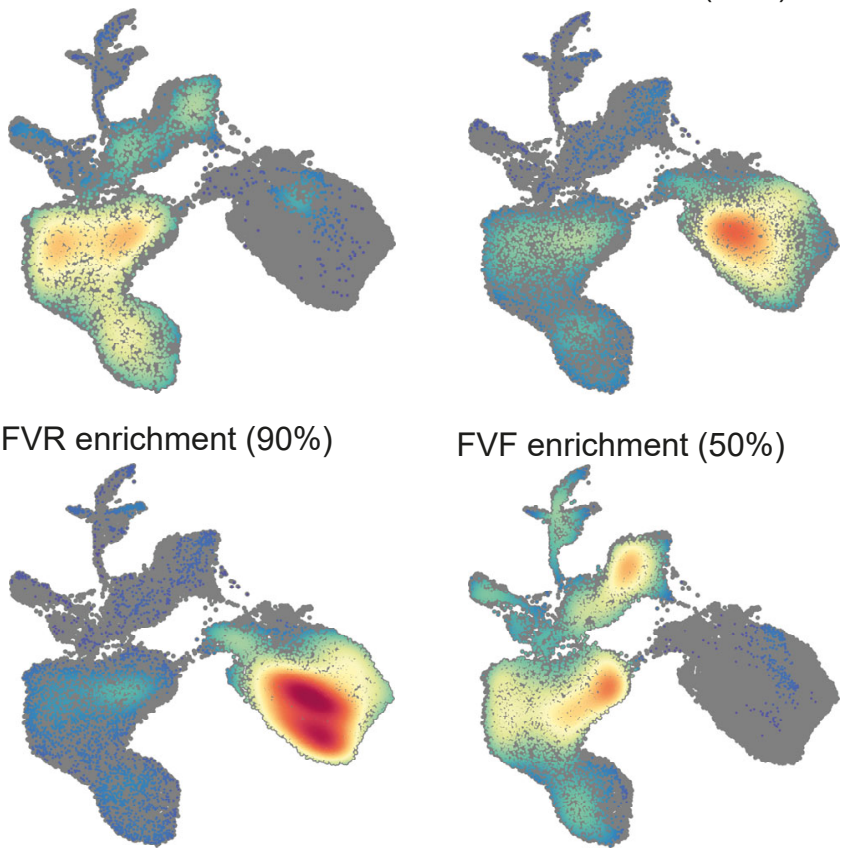

FVF enrichment (50\%)
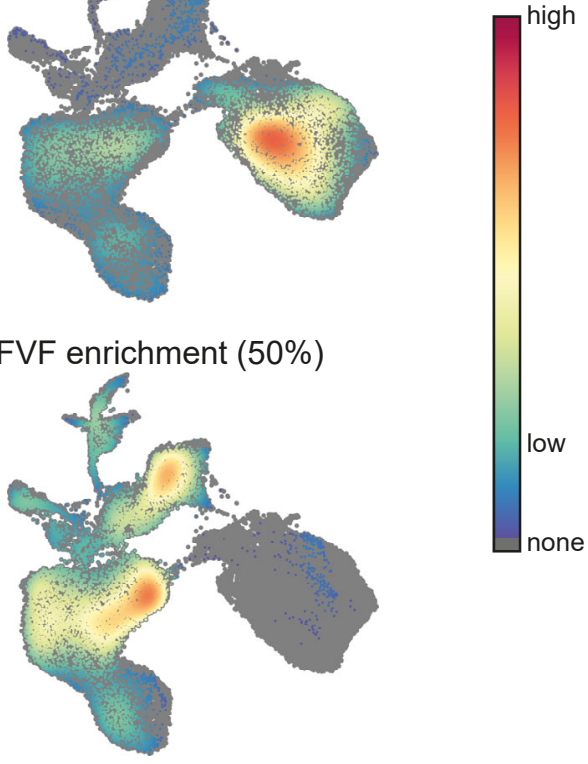

C

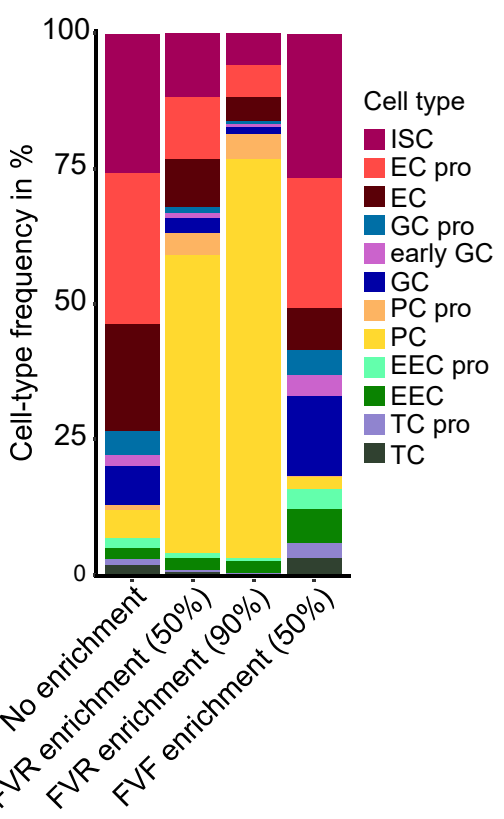

d

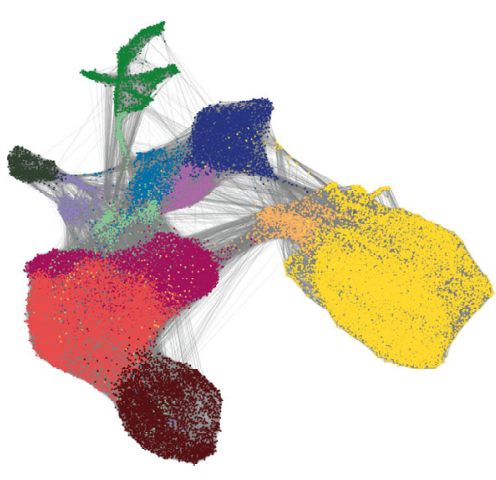

- ISC

- EC pro

- EC

- GC pro

early GC

- GC

PC pro

- PC

EEC pro

- EEC

TC pro

- TC

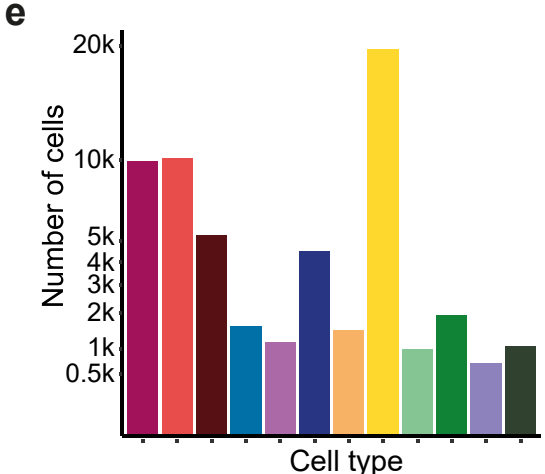


Figure 3 Subtype enrichment of crypt cells reveals distinct progenitor states for all intestinal lineages.

a, Experimental overview. SI crypt cells were obtained from reporter (FVR, FVF) mice to enrich rare cell states, and wild-type mice. Fluorescence-activated cell sorting (FACS) was used to enrich rare crypt cell populations from reporter mice. Transcriptional profiling of single cells was performed using the $10 \mathrm{X}$ Genomics platform.

b, Reporter mice enable the identification of rare cell states. Cell-density plots of FVR and FVF based enrichment compared to a non-enriched sample. No enrichment (pooled cells from 2 mice), FVR enrichment 50\% (pooled cells from 2 mice, flow-enriched $\mathrm{FVR}^{+}$cells were mixed with nonenriched cells in a ratio of 1:2), FVR enrichment 90\% (pooled cells from 2 mice, flow-enriched $\mathrm{FVR}^{+}$cells were mixed with non-enriched cells in a ratio of 9:1), FVV enrichment 50\% (n (mice) $=3$, flow-enriched $\mathrm{FVF}^{+}$cells were mixed with non-enriched cells in a ratio of 1:2.

c, Bar plot depicting the relative abundance of progenitor and mature cell types in the nonenriched, and FVR and FVF enriched sample. FVR enrichment enables the isolation of rare PC progenitors and mature PCs whereas FVF enrichment enables the efficient extraction of the EEC and goblet cell lineage compared to the non-enriched sample. ISC, intestinal stem cell. EC pro, enterocyte progenitor. EC, enterocyte. GC pro, goblet cell progenitor. Early GC, early goblet cell. GC, mature goblet cell. PC pro, Paneth cell progenitor. PC, Paneth cell. EEC pro, enteroendocrine cell progenitor. EEC, enteroendocrine cell. TC pro, tuft cell progenitor. TC, tuft cell.

d, UMAP plots of all control intestinal crypt cells highlighting progenitor cell-type annotation. Grey lines depict 30 nearest neighbors for each cell. Cells were obtained from 10 samples including wild-type (control) and reporter mice for lineage enrichment (Supplementary Table 2).

e, The bar plot depicts the number of captured cells per cell type. 


\section{Böttcher et al. Figure 4}

Temporal-resolved lineage labelling and pseudotemporal ordering of intestinal crypt cells shows that EECs and PCs directly allocate from ISCs via unipotent transition states.

a

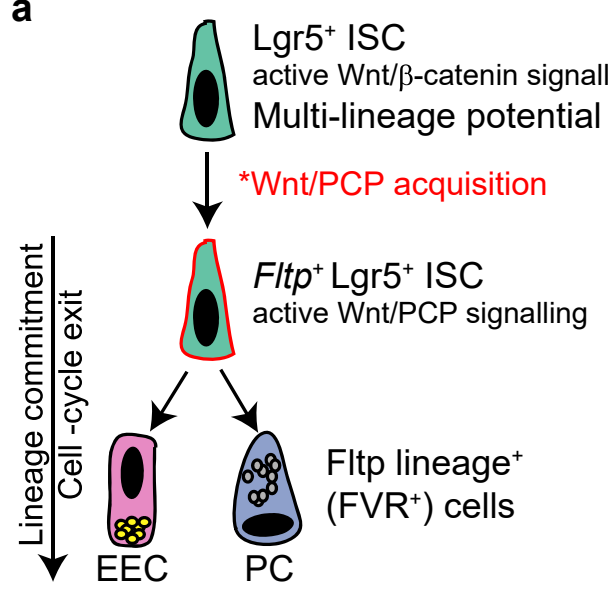

b

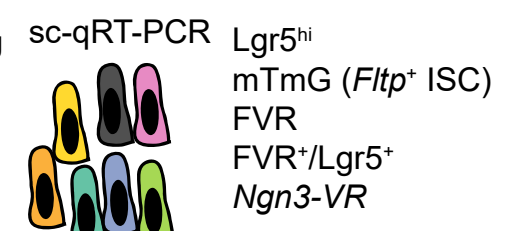

- Stem-cell markers

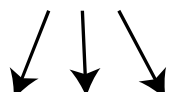

- Intestinal cell-type markers

- Cell-cycle activity/proliferation/ cell-cycle exit

- Notch signalling

- Wnt/ $\beta$-catenin signalling

- Wnt/PCP signalling

- ECM signalling

- Epigenetic regulation

C

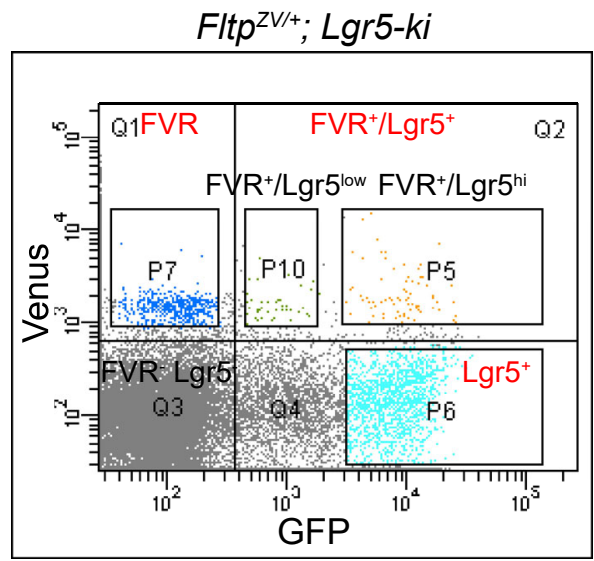

d

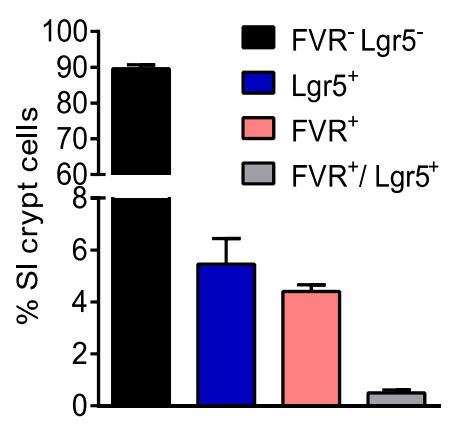

g

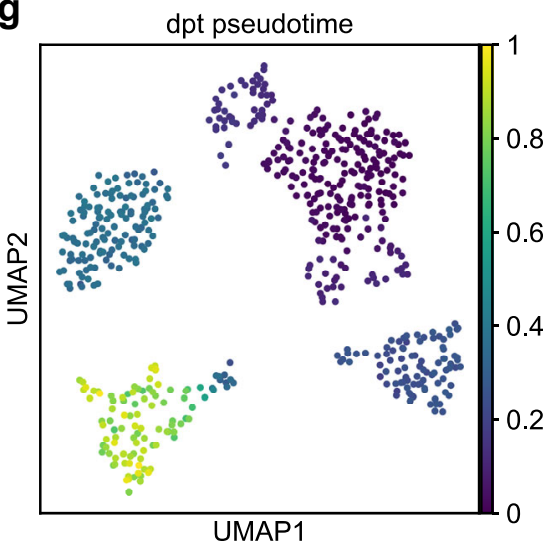

e

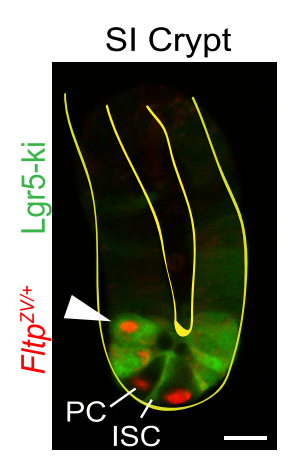

f

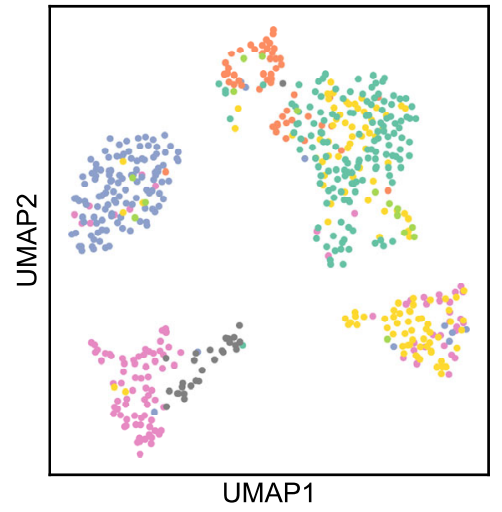

FACS group

FVR ${ }^{\text {low }}$

FVR ${ }^{\text {hi }}$

$\mathrm{FVR}^{+} / \operatorname{Lgr} 5^{\mathrm{hi}}$

mTmG

Lgr5 ${ }^{\text {hi }}$

Ngn3-VF

$\mathrm{FVR}^{+} /$Lgr5low h

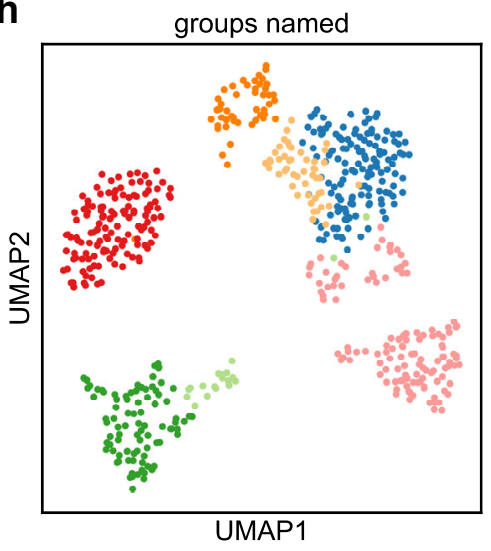

i

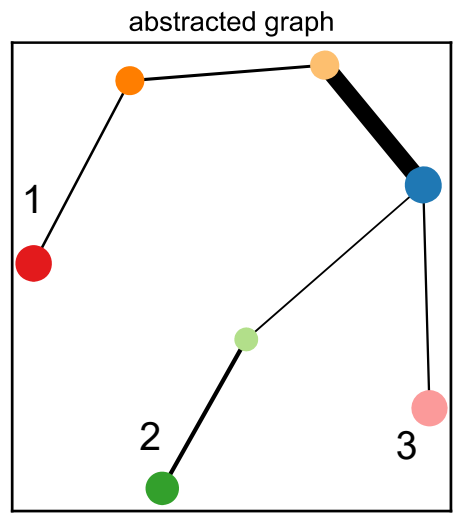

j

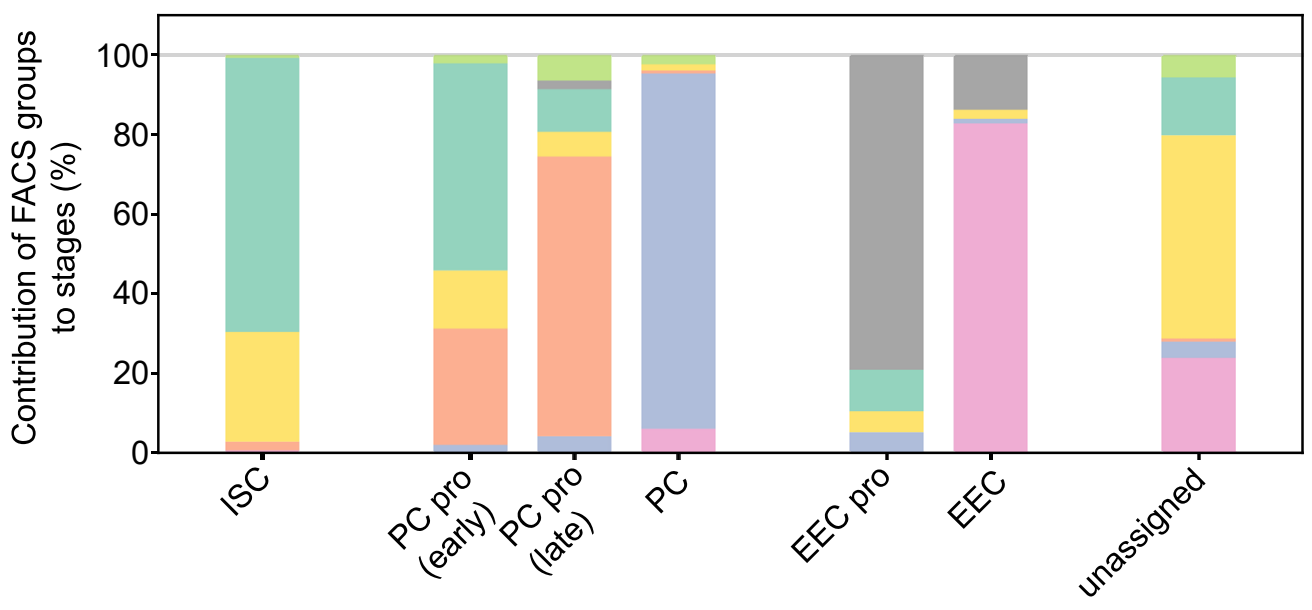

FACS group

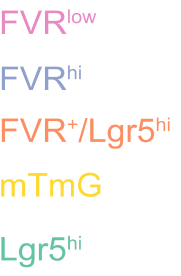

Ngn3-VF 
Figure 4 Temporal-resolved lineage labelling and pseudotemporal ordering of intestinal crypt cells shows that EECs and PCs directly allocate from ISCs via unipotent transition states.

a, b, Wnt/PCP activated Fltp ${ }^{+}$ISCs are committed to differentiate into Paneth cells (PCs) and enteroendocrine cells (EECs) (a). Integration of lineage labelling and single-cell gene expression to elucidate the differentiation trajectories from ISCs into the Paneth and enteroendocrine lineage by single-cell qRT-PCR analysis of 80 genes from defined categories in $\operatorname{Lgr}^{\text {hi }}$ ISCs, mTmG cells, FVR cells, $\mathrm{FVR}^{+} / \mathrm{Lgr}^{+}$cells and Ngn3-VR cells (b).

c, d, FACS plot of crypt cells from Fltp ${ }^{Z V /+}$; Lgr5-ki dual reporter mice depicting the separation of rare $\mathrm{FVR}^{+} / \operatorname{Lgr}^{+}\left(\mathrm{FVR}^{+} / \operatorname{Lgr} 5^{\mathrm{hi}}, \mathrm{FVR}^{+} / \operatorname{Lgr} 5^{\text {low }}\right)$ cells from $\mathrm{Lgr}^{+}-\mathrm{GFP}$ and $\mathrm{FVR}$ single positive, and Lgr5-GFP and FVR negative cells (c) and quantification of FACS analysis (d). $0.5 \%$ of the crypt cells are $\mathrm{FVR}^{+} \mathrm{Lgr}^{+}$double positive. $n$ (independent experiments, mice) $=7$. Error bars represent SEM.

e, LSM live image of a representative SI crypt, cultured in matrigel, isolated from Fltp ${ }^{Z V /+}$; Lgr5ki mice showing rare FVR (red), Lgr5 (green) double positive cells (arrowhead) located at position +4 (= supra-Paneth cell position). PC, Paneth cell. ISC, intestinal stem cell (Lgr5 ${ }^{+}$). Scale bar, 10 $\mu \mathrm{m}$.

f, $\mathbf{g}, \mathbf{h}, \mathrm{UMAP}$ projection of $\mathrm{Lgr} 5^{\mathrm{hi}}$ cells, early and late $\mathrm{mTmG}$ cells, $\mathrm{FVR}^{+} / \mathrm{Lgr}^{+}$cells, FVR cells and Ngn3-VF endocrine progenitors based on the expression of 80 marker genes. Each dot represents a single cell. Colours indicate FACS groups (f), pseudotime computed using dpt (g), and cell-type clusters annotated based on marker genes (h).

i, PAGA plot showing relationship of cell type clusters in (h).

j, The bar plot depicts the contribution of FACS groups to stages in the PC and EEC branch. 


\section{Böttcher et al. Figure 5}

FVR marks Lgr5+ label-retaining cells that give rise to Paneth cells.

a

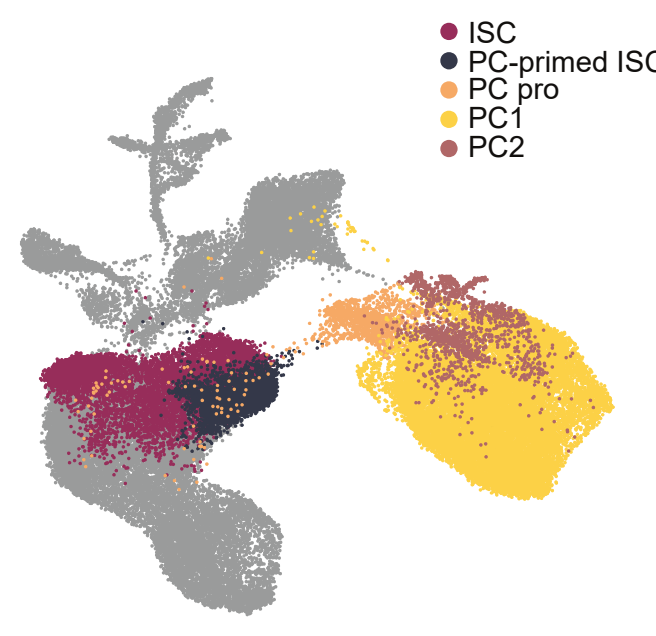

b

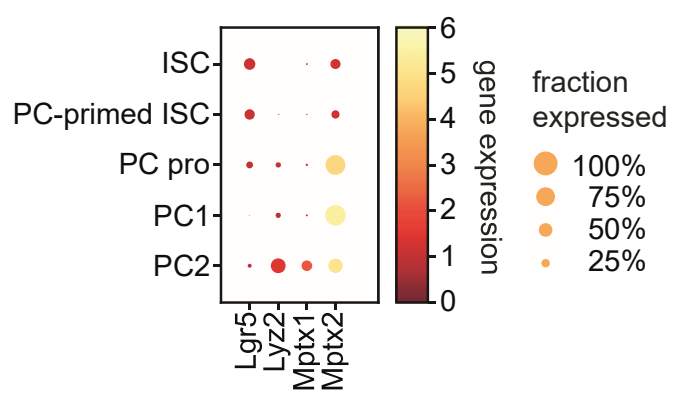

e

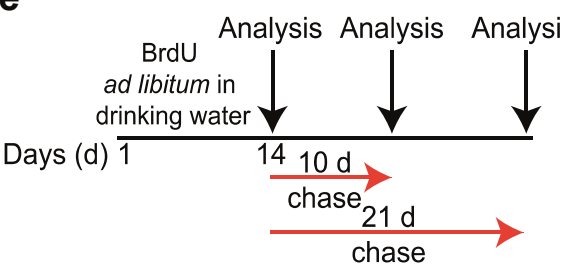

f

$14 \mathrm{~d}$ BrdU administration
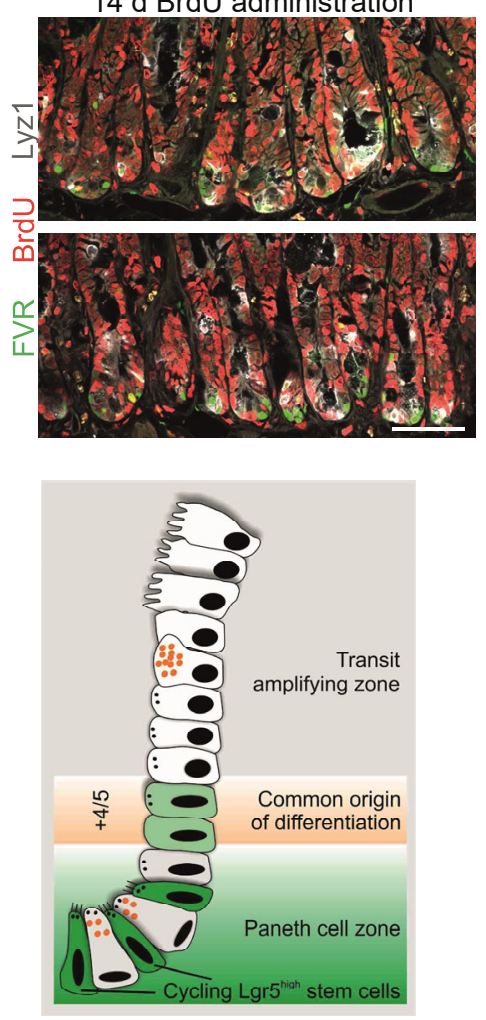

j
C
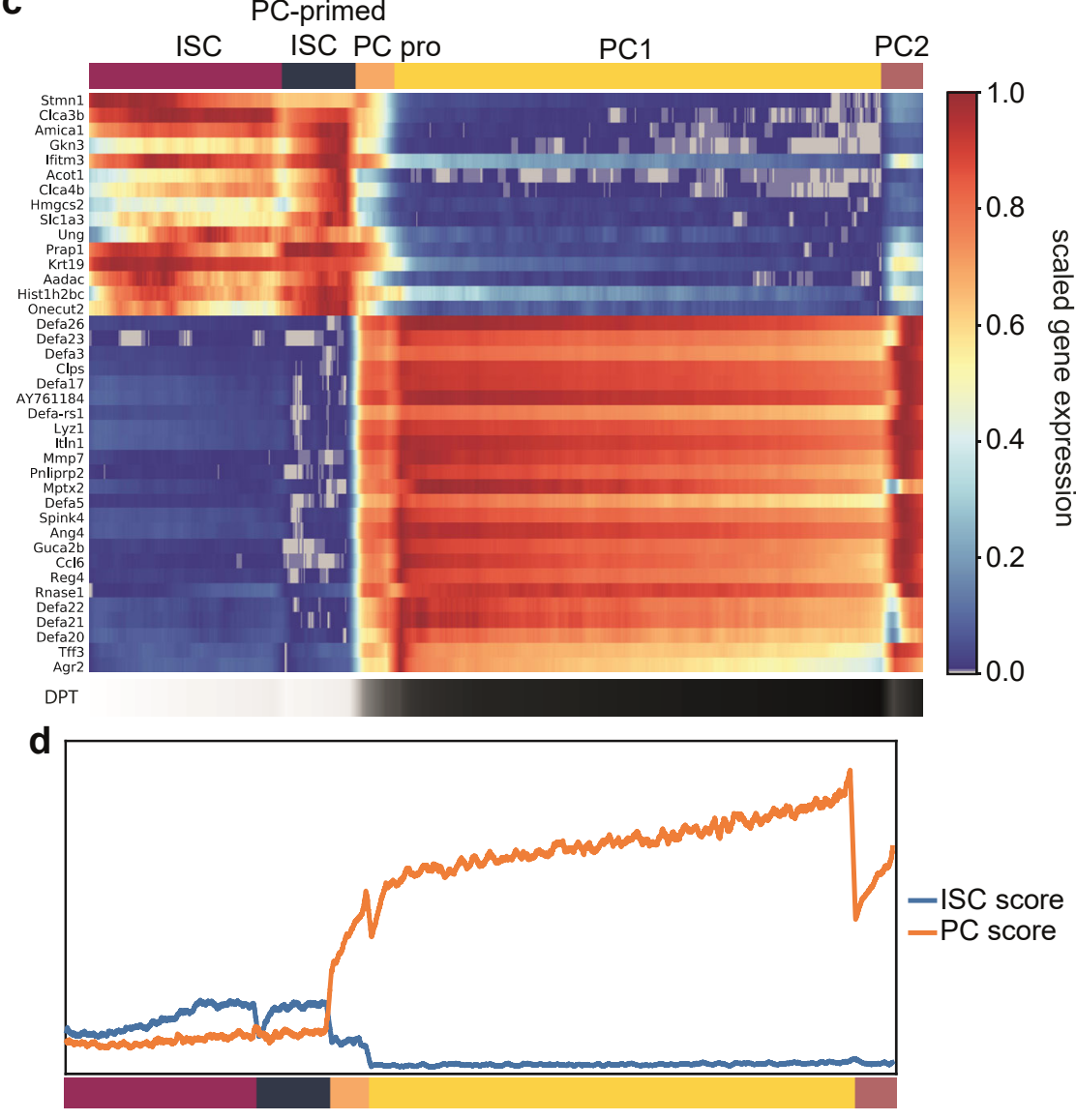

h
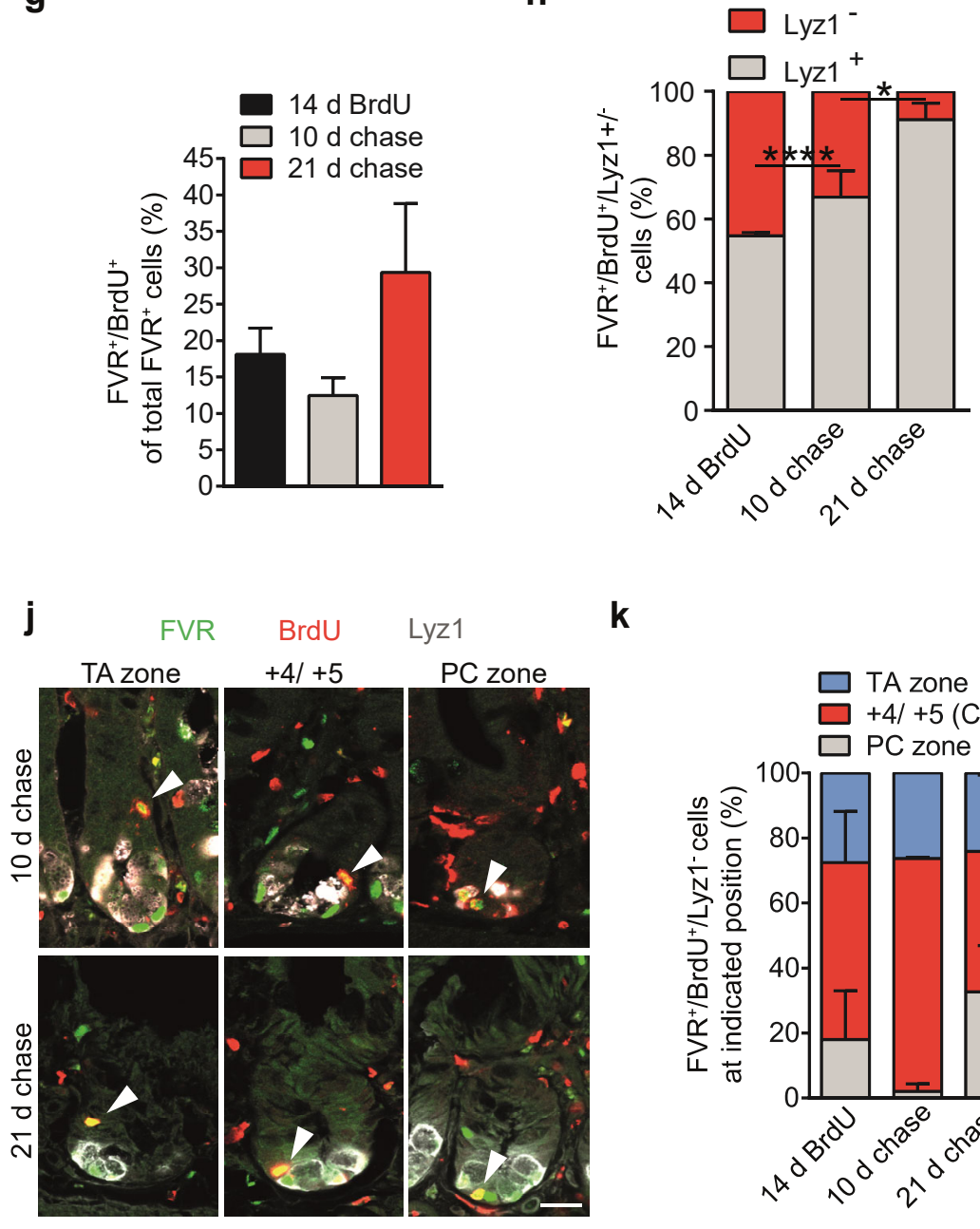

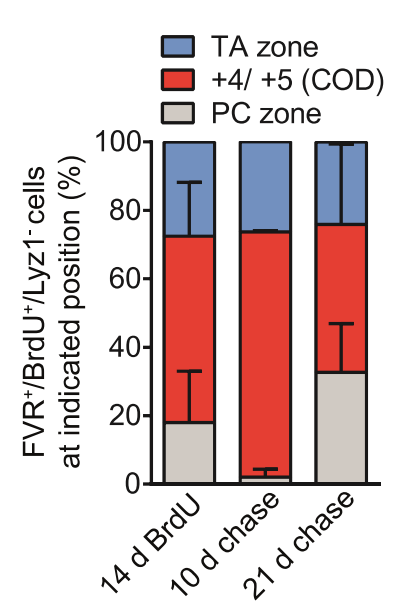


Figure 5 FVR marks Lgr5 ${ }^{+}$label-retaining cells that give rise to Paneth cells.

a, UMAP plot depicting the identified cell states during differentiation of ISCs towards the Paneth cell lineage. Our analysis revealed an ISC subpopulation that is primed towards PC fate (PCprimed ISC), a PC progenitor population (PC pro), a PC population characterized by absence of $L y z 2$ expression (PC1) and a PC population characterized by Lyz2 expression (PC2).

b, Dotplot showing the expression of the stem-cell marker Lgr5 in PC progenitor populations and genes that distinguish the mature PC types PC1 and PC2.

c, Smoothed gene expression heatmap of differentially expressed genes of the displayed cell states plotted along diffusion pseudotime from ISCs to mature PCs.

d, ISC score and PC score along pseudotime shows high ISC score in PC-primed ISCs. Also, Paneth cell progenitors express both ISC and PC markers.

e, Experimental scheme of the 5-bromo-2'-deoxyuridine (BrdU) pulse-chase experiment. To assess label-retention, Fltp ${ }^{Z V /+}$ mice were treated with BrdU for 14 days (d) and analysed after a chase period of 10 and $21 \mathrm{~d}$.

f, Representative LSM images of duodenal sections stained for FVR (Venus, green), BrdU (red) and Lyz1 (white, Paneth cells) after $14 \mathrm{~d}$ BrdU administration. Scale bar, $25 \mu \mathrm{m}$.

g, Relative proportion of $\mathrm{FVR}^{+} / \mathrm{BrdU}^{+}$cells of total $\mathrm{FVR}^{+}$cells at the indicated time points. $14 \mathrm{~d}$ BrdU: $n$ (mice) $=4$ with 1432 analysed FVR ${ }^{+}$cells; $10 \mathrm{~d}$ chase: $n$ (mice) $=2$ with 785 analysed FVR ${ }^{+}$cells; 21 d chase: $n$ (mice) $=4$ with 2401 analysed $\mathrm{FVR}^{+}$cells. Error bars represent SD.

h, Relative proportion of Lyz1 ${ }^{+}$(long-living and newly formed Paneth cells) and Lyz1- (LRC) cells of the total $\mathrm{FVR}^{+} / \mathrm{BrdU}^{+}$cells at the indicated time points. $14 \mathrm{~d}$ BrdU: $n$ (mice) $=4$ with 1432 analysed FVR ${ }^{+}$cells; 10 d chase: $n$ (mice) $=2$ with 785 analysed FVR ${ }^{+}$cells; $21 \mathrm{~d}$ chase: $n$ (mice) $=4$ with 2401 analysed $\mathrm{FVR}^{+}$cells. Error bars represent SD. Two-tailed Student's $t$-test, $* P<0.05$, $* * * * P<0.0001$.

$\mathbf{i}, \mathbf{j}, \mathbf{k}$, Scheme depicting the compartmentalization of the SI crypt. Actively cycling stem cells $\left(\mathrm{Lgr5}^{+}\right)$and Paneth cells reside in the Paneth cell (PC) zone. Quiescent/label-retaining cells locate at position $+4 /+5$. The transit-amplifying zone (TA zone) contains mainly proliferative 
(absorptive) progenitors (i). Representative LSM images from duodenal sections stained for FVR (Venus, green), BrdU (red) and Lyz1 (white, Paneth cells) after $10 \mathrm{~d}$ and $21 \mathrm{~d}$ chase. The position (arrowhead) of the $\mathrm{FVR}^{+} \mathrm{LRC}\left(\mathrm{BrdU}^{+} / \mathrm{Lyzl}^{-}\right)$is defined according to the scheme in (i). Quantification of $\mathrm{FVR}^{+} \mathrm{LRCs}\left(\mathrm{BrdU}^{+} / \mathrm{Lyzl}^{-}\right.$) at indicated positions (according to j). $14 \mathrm{~d} \mathrm{BrdU:} n$ $($ cells $)=116$ (in 4 mice), $10 \mathrm{~d}$ chase: $n($ cells $)=32$ (in 2 mice), $21 \mathrm{~d}$ chase: $n$ (cells) $=55$ (in 4 mice) (k). Scale bar, $25 \mu \mathrm{m}(\mathbf{j})$. Error bars represent SD. 


\section{Böttcher et al. Extended Data Figure 1}

FVR labels all Paneth and enteroendocrine subtypes.

a

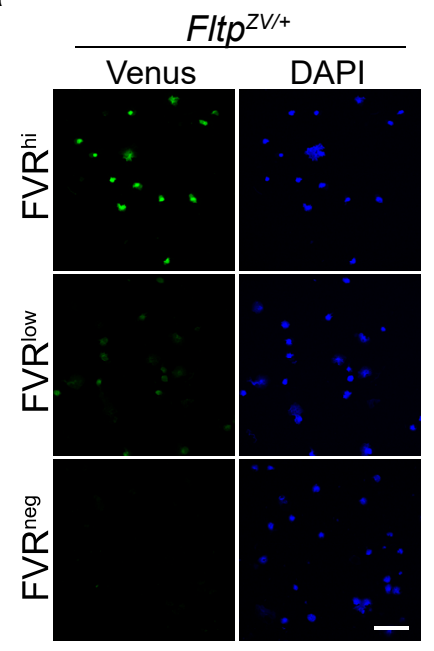

d Lgr5-EGFP-iresCreERT2 (Lgr5-ki) reporter

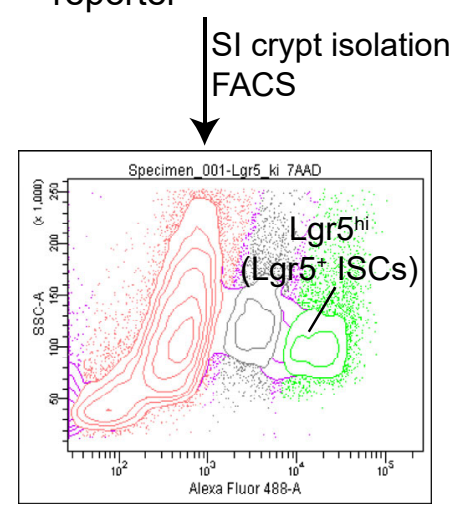

g

EEC genes

$F R^{\text {neg }} F R^{\text {low }} F V R^{\text {hi }}$

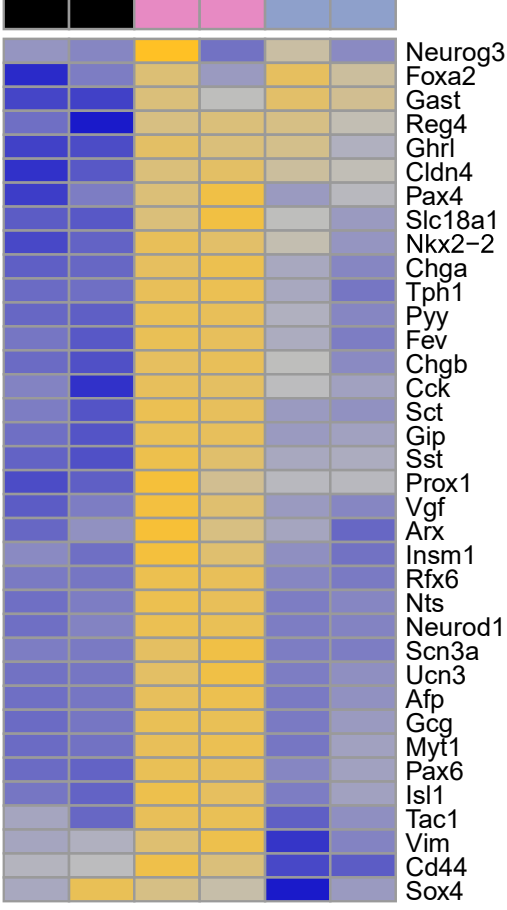

C Fltp $^{Z \mathrm{~V} /+}$

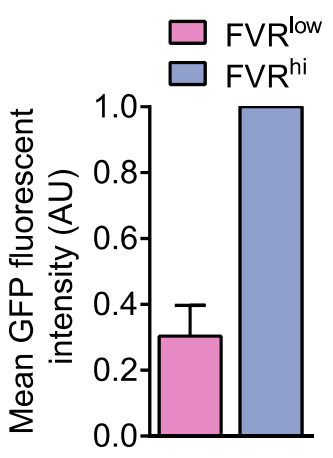

e

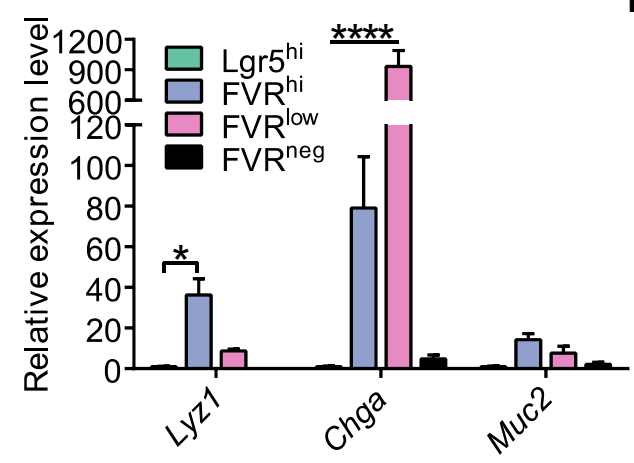

h PC via Lgr5+ ISCs

(Grün et al., 2016)

FVR $^{\text {neg }}$ FVR $^{\text {low }}$ FVR $^{\text {hi }}$

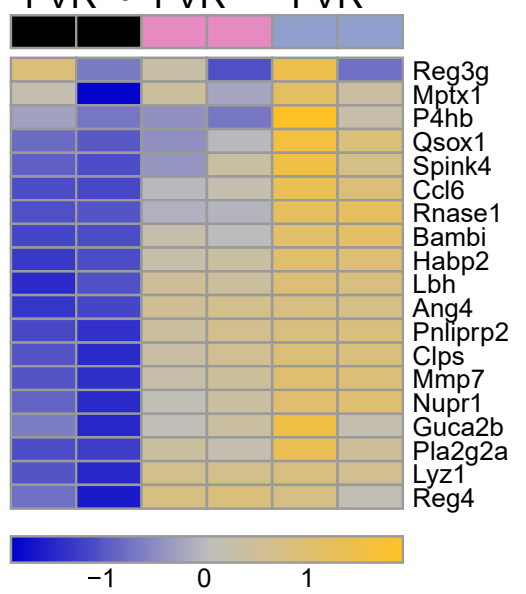

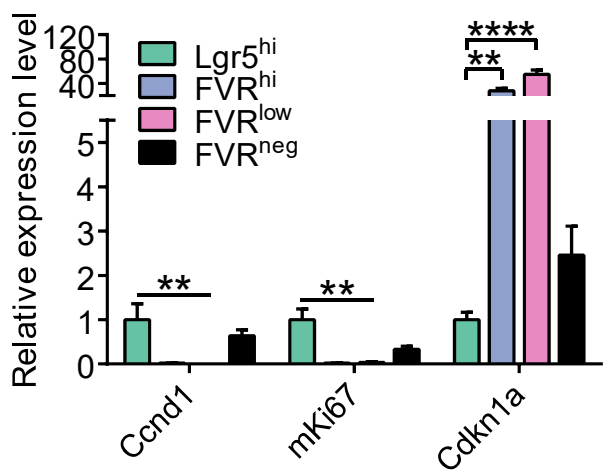

PC via Goblet/Paneth cell precursor (Grün et al., 2016)

FVR $^{\text {neg }} F V^{\text {low }} F^{\text {FV }}{ }^{\text {hi }}$

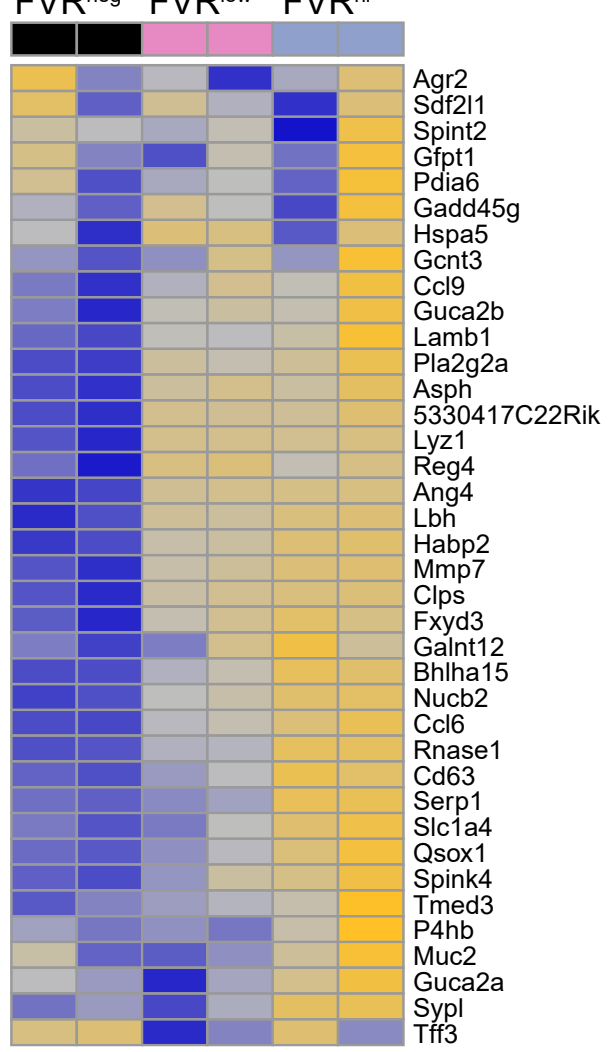


Extended Data Figure 1 FVR labels all Paneth and enteroendocrine subtypes.

a, LSM images of immune-stained flow-sorted FVR ${ }^{\text {hillow/neg }}$ SI crypt cells isolated from Fltp ${ }^{Z V /+}$ mice showing that three distinct crypt cell populations are distinguishable by FVR activity. FVR (Venus, green), DAPI (blue, nuclei). Scale bar, $75 \mu \mathrm{m}$.

b, Relative mean fluorescent intensity of GFP (Venus) in $\mathrm{FVR}^{\text {low }}$ and $\mathrm{FVR}^{\text {hi }}$ cells isolated from Fltp ${ }^{Z V /+}$ cells by flow cytometry. $n$ (independent experiments) $=6$. Error bar represent SD.

c, Heatmap depicting the p-values determined by Fisher's exact test of Kyoto Encyclopedia of Genes and Genomes (KEGG) pathways which were significantly enriched in at least one of the populations. Pathways associated with neuroendocrine (green), immuno-regulatory function (blue), actin cytoskeletal regulation and signalling (MAPK/ Insulin, red) as well as cellular metabolism (black) and the cell cycle (red) are indicated.

d, FACS plot depicting the experimental strategy to obtain $\mathrm{Lgr} 5^{\text {hi }}$ cells (intestinal stem cells, ISCs) from Lgr5-EGFP-ires-CreERT2 (Lgr5-ki) mice.

e, f, Confirmation of the microarray results. Comparison of the crypt cell populations distinguishable by FVR activity with Lgr5 $5^{\text {hi }}$ ISCs by qRT-PCR, for the expression of intestinal lineage markers (Lyz1, Paneth cells; Chga, enteroendocrine cells; Muc2, goblet cells) (e) and proliferation markers $(C c n d 1, K i 67)$ and cell-cycle exit marker $(C d k n 1 a)$ (f). $n$ (independent experiments $)=3$. Error bars represent SEM. Two-tailed Student's $t$-test, ${ }^{*} P<0.05,{ }^{* *} P<0.01$, $* * * * P<0.0001$.

$\mathbf{g}, \mathbf{h}, \mathbf{i}$, Heatmaps depicting the enrichment of the enteroendocrine cell (EEC) gene signature in the $\mathrm{FVR}^{\text {low }}$ population (g), and the enrichment of genes expressed in Paneth cells (PC) formed via Lgr $5^{\text {hi }}$ ISCs (h) or via a Goblet/Paneth cell precursor (i) in FVR ${ }^{\text {hi }}$ cells. Gene signatures are derived from Grün et al., 2016. FVR labels all reported EEC subsets ${ }^{29}$. Expression is scaled row-wise and the colours range from dark blue (low expression) to orange (high expression) and represent normalized expression (row z-score). 


\section{Böttcher et al. Extended Data Figure 2}

Fltp ${ }^{+}$cells possess limited organoid forming capacity in vitro.

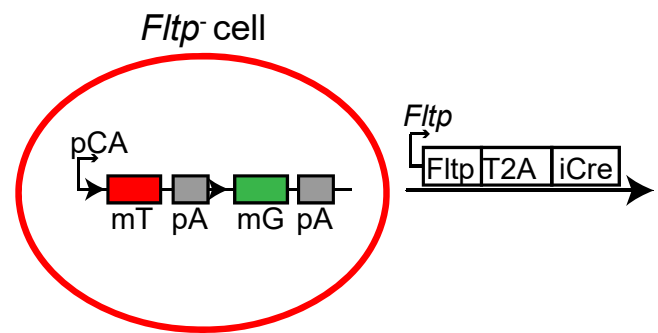

Fltp $^{+}$cell $=m T m G$

Fltp lineage ${ }^{+}$cell

b
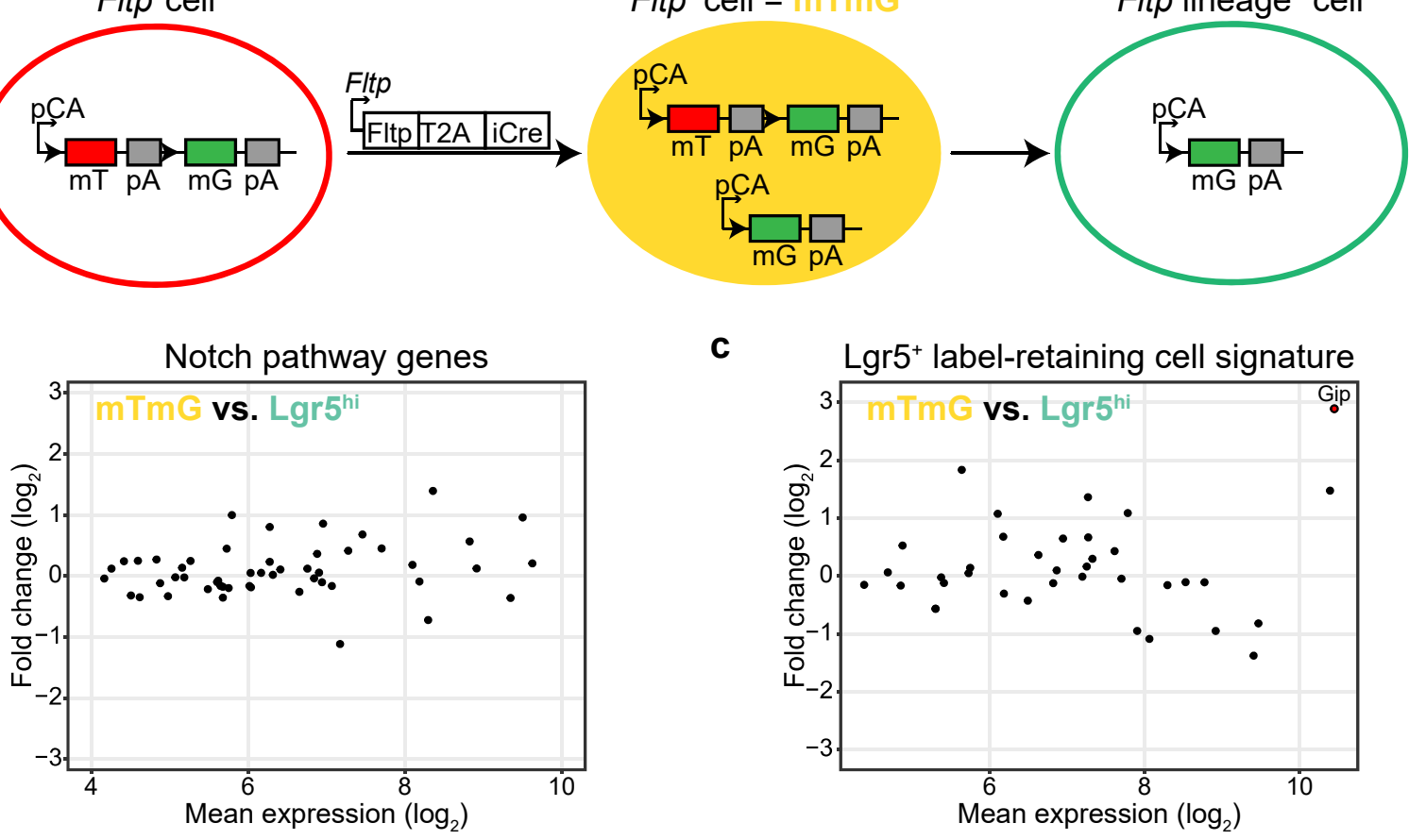

- Differentially expressed genes $(F D R<0.01)$

- Similarily expressed genes (FDR $>0.01$ )

C

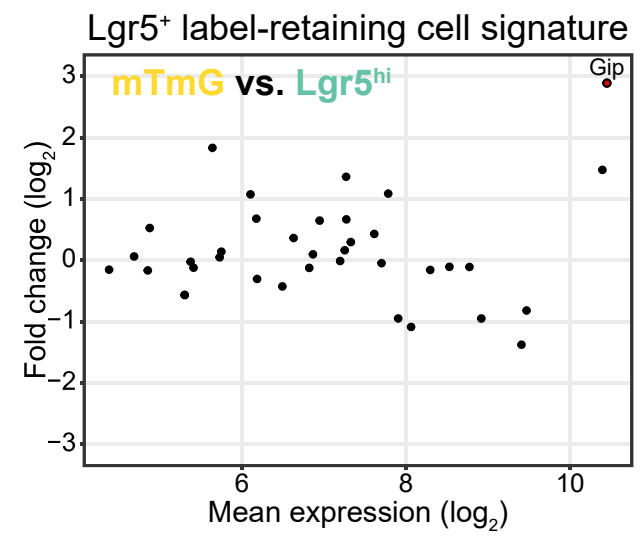

- Differentially expressed genes $(F D R<0.01)$

- Similarily expressed genes (FDR $>0.01$ )

d

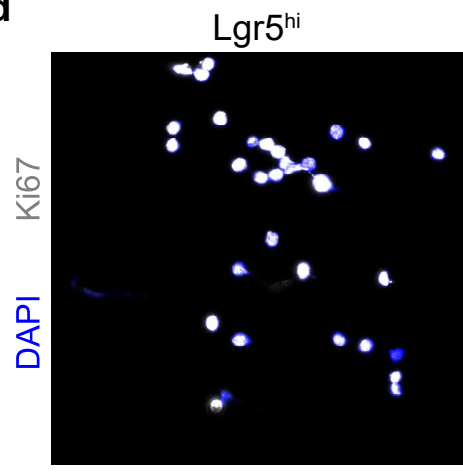

f

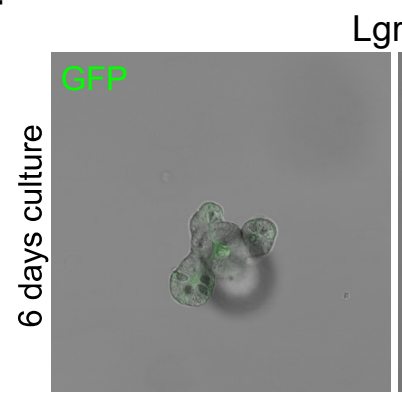

$\operatorname{Lgr}^{\text {hi }}$

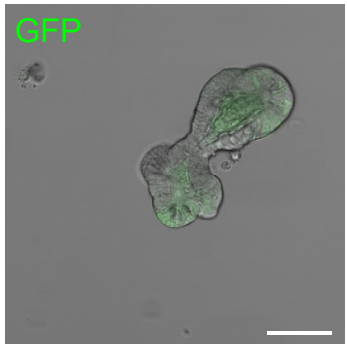

$\mathrm{mTmG}$

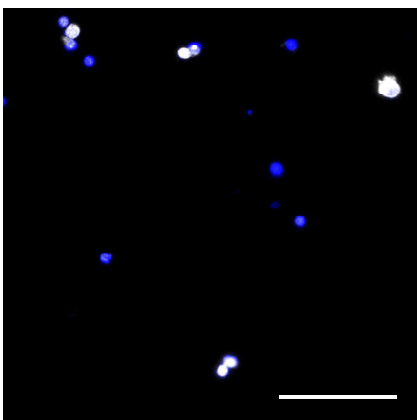

e

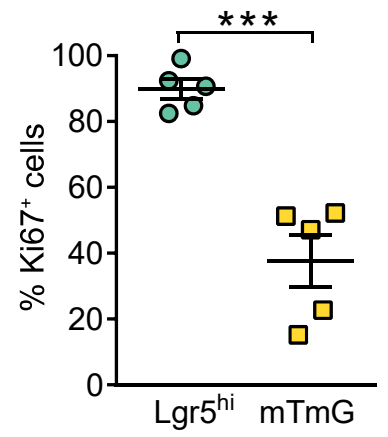

g $\mathrm{mTmG}$

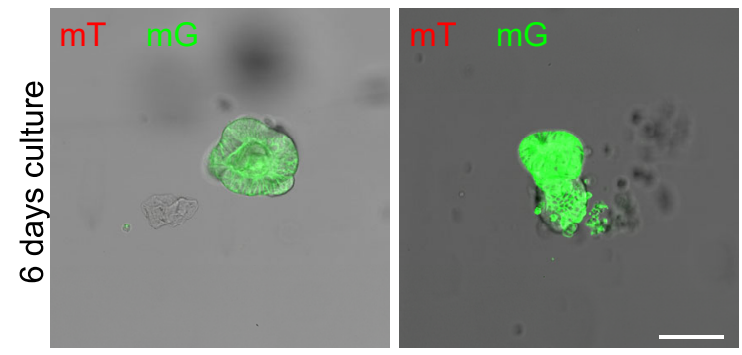

h

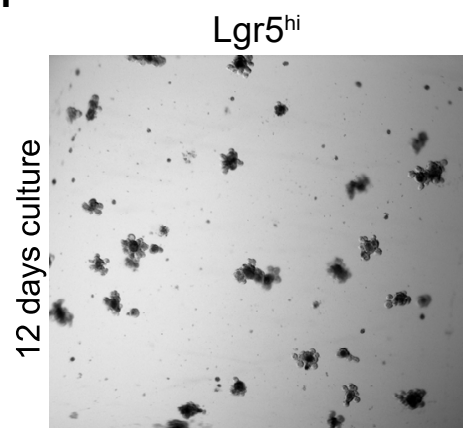

$\mathrm{mTmG}$

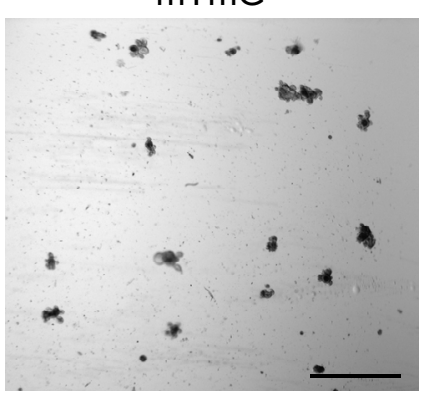

i

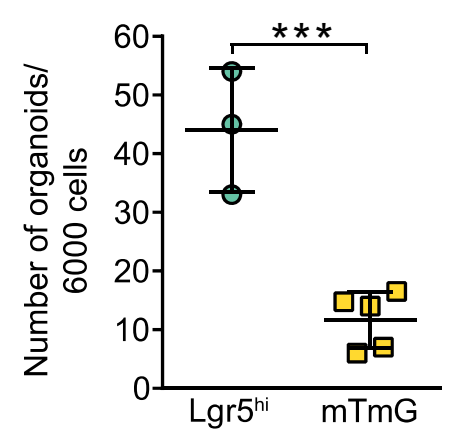


Extended Data Figure $2 \mathrm{Fltp}^{+}$cells possess limited organoid forming capacity in vitro.

a, Schematic depicting the Fltp ${ }^{T 2 A i C r e /+}$; Gt(ROSA) $26^{m T m G /+}$ lineage-tracing model. Fltp lineage cells (mT, red) convert into Fltp lineage ${ }^{+}$cells $(\mathrm{mG}$, green) upon Fltp-promoter driven Cre expression via an intermediate ( $\mathrm{mTmG}$, yellow) cell state. $\mathrm{mTmG}$ cells represent cells that started to express Fltp.

b, MA-plot comparing the expression of Notch pathway genes (KEGG mmu 04330) in mTmG and Lgr $5^{\text {hi }}$ (ISCs) cells. The y-axis depicts the fold change in $\log 2$ and the $\mathrm{x}$-axis depicts the mean $\log 2$ expression value. No gene is significantly regulated. $n$ (microarray samples) $=6$ for Lgr $5^{\text {hi }}$ ISC. $n$ (microarray samples) $=4$ for $\mathrm{mTmG}$.

c, MA-plot comparing the expression of Lgr $^{+}$label-retaining cell signature genes ${ }^{7}$ in $\mathrm{mTmG}^{\mathrm{T}}$ and Lgr $^{\text {hi }}$ (ISCs) cells. Differentially expressed genes $(F D R<0.01)$ are shown in red. The y-axis depicts the fold change in $\log 2$ and the x-axis depicts the mean $\log 2$ expression value. Gip is the only significantly regulated gene. $n$ (microarray samples) $=6$ for Lgr $5^{\text {hi }}$ cells. $n$ (microarray samples) $=4$ for $\mathrm{mTmG}$.

d, e, Representative LSM images showing Lgr $5^{\text {hi }}$ and mTmG cells stained for Ki67 (white) and DAPI (blue, nucleus) (d) and quantification of Ki67 positive cells (e). $n$ (mice) $=5$ for Lgr $5^{\text {hi }}$ cells. $n$ (mice) $=5$ for $\mathrm{mTmG}$ cells. Error bars represent SEM. Two-tailed Student's $t$-test, ${ }^{* * *} P<0.001$. Scale bar, $75 \mu \mathrm{m}$.

f, g, Representative LSM images showing live organoids derived from single Lgr $5^{\text {hi }}$ ISCs (f) and $\mathrm{mTmG}$ cells $(\mathbf{g})$. Scale bars, $75 \mu \mathrm{m}$.

h, i, Representative bright-field images of 6,000 FACS-purified mTmG and Lgr $5^{\text {hi }}$ cells cultured for 12 days (h) and quantification of organoid number (i). $n$ (independent experiments) $=3$ for Lgr $5^{\text {hi }}$ cells. $n$ (independent experiments) $=5$ for $\mathrm{mTmG}$ cells. Error bars represent SD. Two-tailed Student's $t$-test, $* * * P<0.001$. Scale bar, $5 \mathrm{~mm}$. 


\section{Böttcher et al. Extended Data Figure 3}

Fltp ${ }^{+}$cells possess limited multi-lineage potential in vivo, are resistant to chemical injury but do not contribute to regeneration after intestinal injury.

a

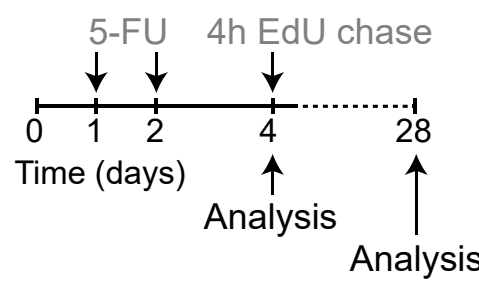

b

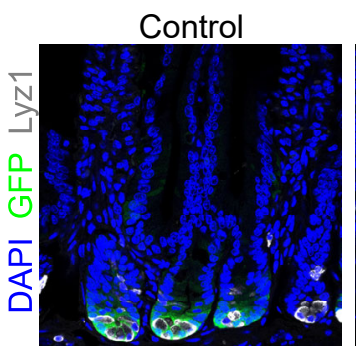

$48 \mathrm{~h}$ post $5-\mathrm{FU}$

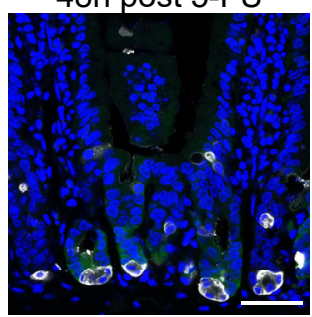

e
C

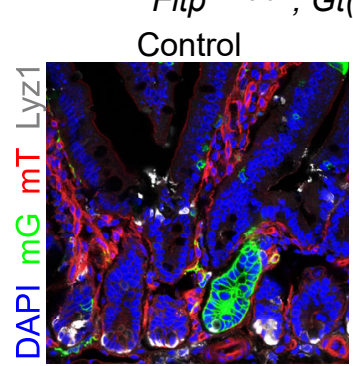

48h post 5-FU

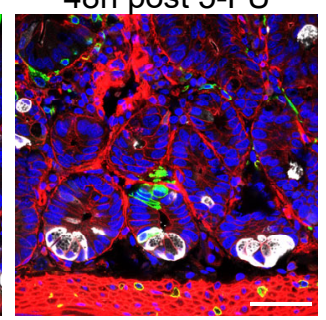

Fltp ${ }^{T 2 A i C r e /+} ; G t(R O S A) 26^{m T m G /+}$ d

Lgr5-EGFP-ires-CreERT2

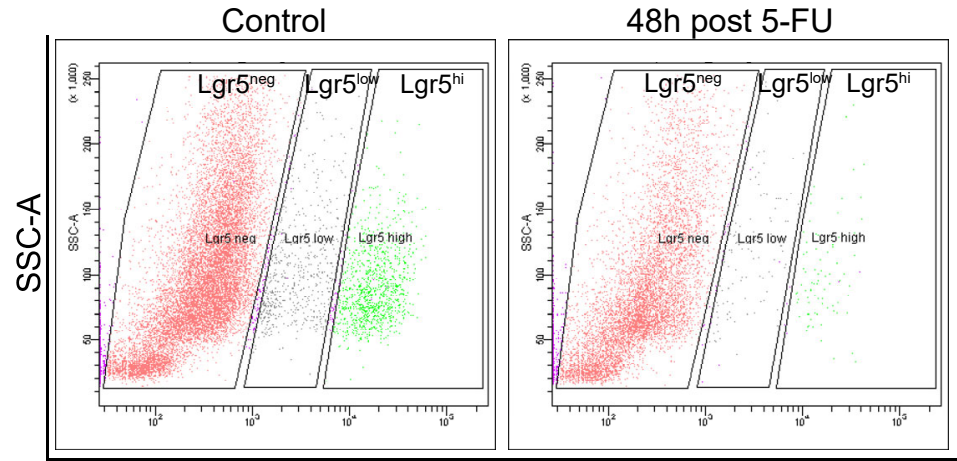

Lgr5::EGFP

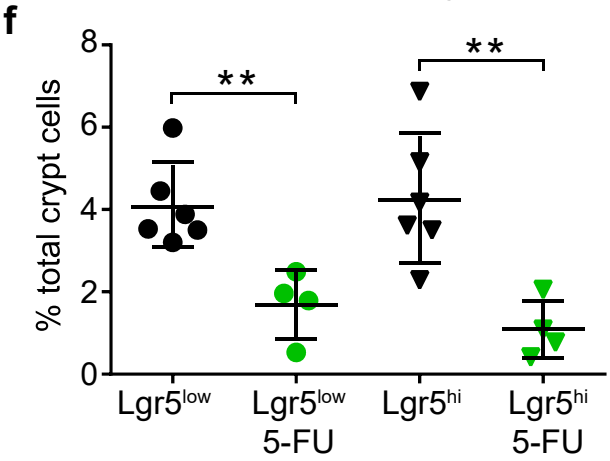

g

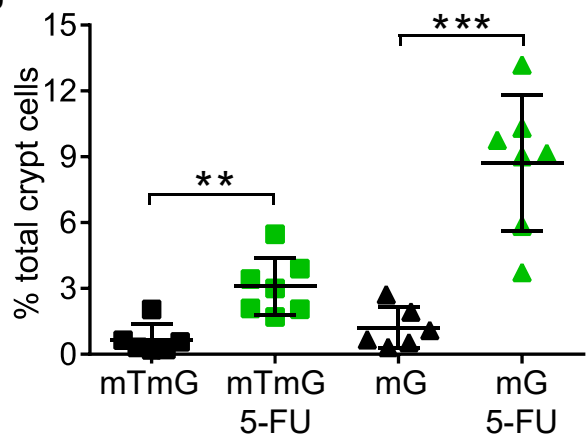

h
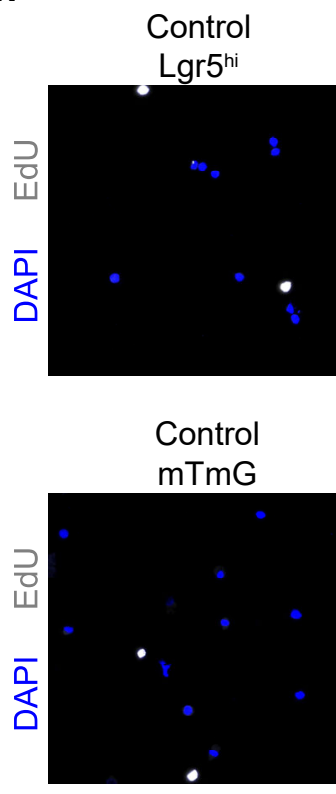
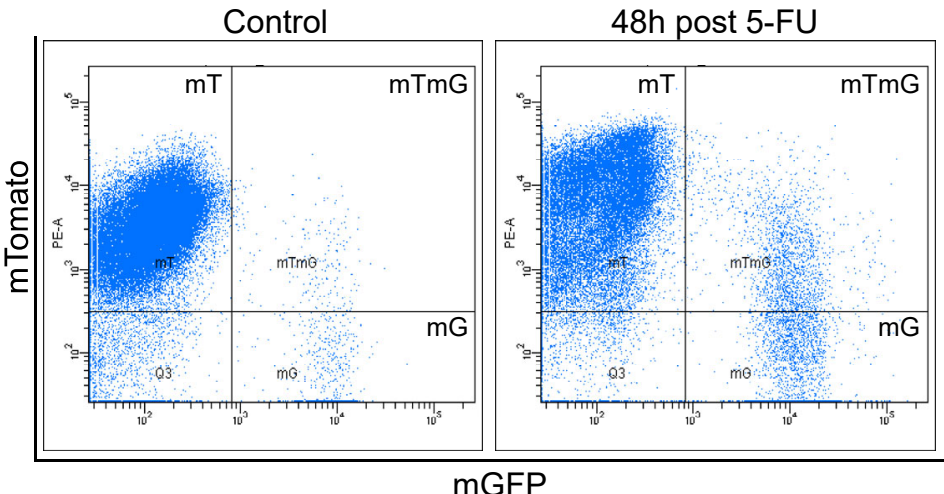

mGFP

5-FU

$\operatorname{Lgr}^{\text {hi }}$

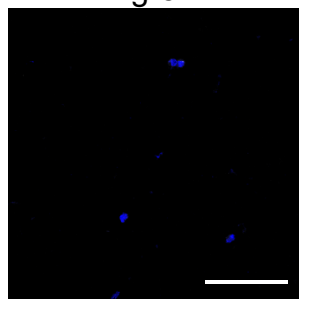

5-FU

$\mathrm{mTmG}$
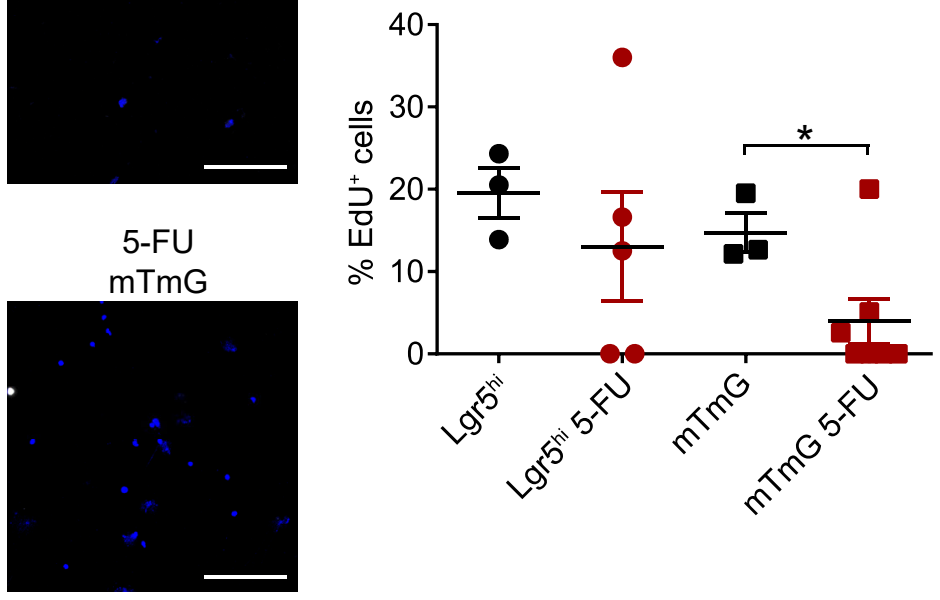

j

Fltp ${ }^{\text {T2AiCre/+; }}$ Gt(ROSA)26 $6^{m T m G /+}$ Control

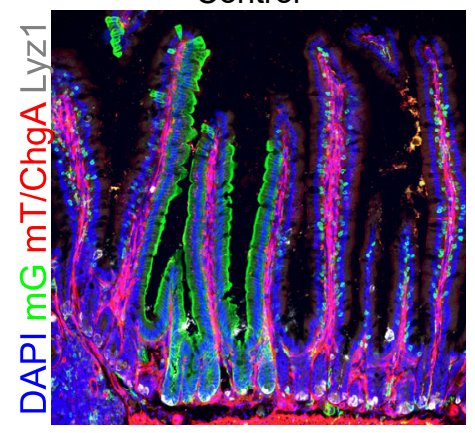

26d post 5-FU

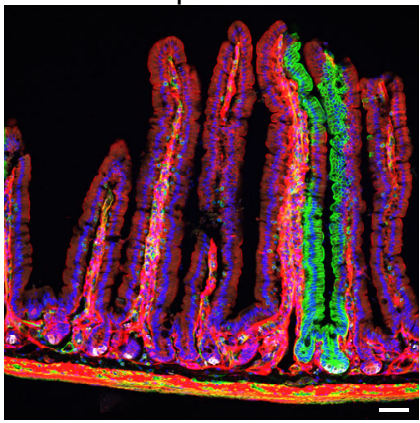

k

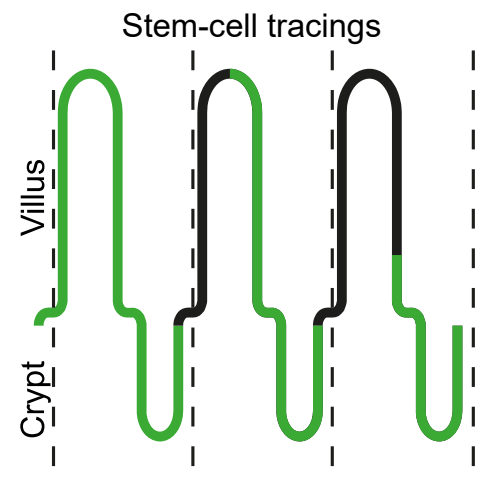

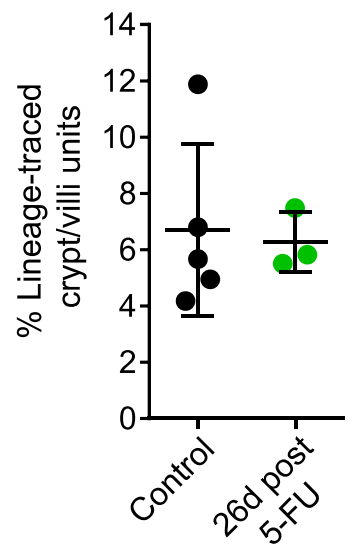


Extended Data Figure $3 \mathrm{Fltp}^{+}$cells possess limited multi-lineage potential in vivo, are resistant to chemical injury but do not contribute to regeneration after intestinal injury.

a, Experimental setup to assess stress response of Fltp $^{+}$cells (mTmG) compared to Lgr5 ${ }^{\text {hi }}$ ISCs. Mice were treated with two consecutive doses of 5-FU $\left(100 \mathrm{mg} / \mathrm{kg} /\right.$ day). Lgr5 ${ }^{+}$cells and Fltp ${ }^{+}$ $\mathrm{mTmG}$ cells were analysed by flow cytometry $48 \mathrm{~h}$ and 24 days after the final 5-FU dose. Proliferative activity was determined after a $4 \mathrm{~h}$ chase of 5-ethynyl-2'-deoxyuridine (EdU) $48 \mathrm{~h}$ after the final 5-FU dose.

b, c, Representative LSM images of duodenal sections from Lgr5-ki (b) and Fltp ${ }^{\text {T2AiCre/+ }}$; Gt(ROSA) $26^{m T m G /+}$ mice (c) $48 \mathrm{~h}$ post 5 -FU treatment. Sections were stained for DAPI (blue, nucleus), GFP (Lgr5 ${ }^{+}$cells, green) and Lyz1 (white, Paneth cells) (b) or mT (Fltp lineage-, RFP, red), $\mathrm{mG}$ (Fltp lineage ${ }^{+}$, GFP, green), and Lyz1 (white, Paneth cells) (c). Scale bars, $75 \mu \mathrm{m}$. $^{\circ}$

d-g, Representative FACS plots of Lgr5-GFP cell populations (d) and $\mathrm{mT} / \mathrm{mTmG} / \mathrm{mG}$ cell populations (e) from untreated and 5-FU treated mice and quantification of frequencies referred to total crypt cells $(\mathbf{f}, \mathbf{g}) 48 \mathrm{~h}$ post 5 -FU treatment. $n$ (mice) $=6$ for untreated Lgr5-ki mice. $n$ (mice) $=4$ for treated Lgr5-ki mice. $n$ (mice) $=6$ for untreated Fltp ${ }^{T 2 A i C r e /+}$; Gt $(\operatorname{ROSA}) 26^{m T m G /+}$ mice. $n$ $($ mice $)=7$ for treated $F l t p^{T 2 A i C r e /+} ;$ Gt $(\operatorname{ROSA}) 26^{m T m G /+}$ mice. Error bars represent SEM. Two-tailed Student's $t$-test, $* * P<0.01, * * * P<0.001$.

h, i, Representative LSM images (h) and quantification (i) of 5-ethynyl-2'-deoxyuridine (EdU) positive (white) Lgr $5^{\text {hi }}$ and $\mathrm{mTmG}$ cells after a $4 \mathrm{~h}$ chase of 5-ethynyl-2'-deoxyuridine (EdU) $48 \mathrm{~h}$ after the final 5-FU dose. DAPI (blue) stains the nucleus. $n$ (mice) $=3$ for untreated Lgr5-ki mice. $n($ mice $)=5$ for treated Lgr5-ki mice. $n$ (mice) $=3$ for Fltp ${ }^{T 2 A i C r e /+}$; Gt $\left(\right.$ ROSA) $26^{m T m G /+}$ mice. $n$ $($ mice $)=7$ for treated Fltp $^{T 2 A i C r e /+} ; G t(R O S A) 26^{m T m G /+}$ mice. Error bars represent SEM. Two-tailed Student's $t$-test, ${ }^{*} P<0.1$. Scale bars, $75 \mu \mathrm{m}$.

$\mathbf{j}, \mathbf{k}, \mathbf{l}$, Representative LSM images showing immune-stained duodenal sections from untreated and 5-FU treated Fltp ${ }^{T 2 A i C r e /+}$; Gt(ROSA) $26^{m T m G /+}$ mice. DAPI (blue) stains nuclei, mGFP (green) labels Fltp lineage ${ }^{+}$cells, mTomato (red) marks Fltp ${ }^{-}$cells, Lyz1 (Paneth cells, white) and ChgA (enteroendocrine cells, red) (j). Scheme showing the mGFP expression pattern in crypt-villi units that are considered as stem-cell tracings (mGFP expression in all intestinal lineages in crypt-villi 
units) (k) and quantification of stem-cell tracings from sections (I). $n$ (mice) $=5$ for untreated mice. $n($ mice $)=3$ for 5 -FU treated mice. Scale bar, $75 \mu \mathrm{m}$. Error bars represent SD. 


\section{Böttcher et al. Extended Data Figure 4}

Identification of progenitors for each intestinal lineage by scRNAseq.

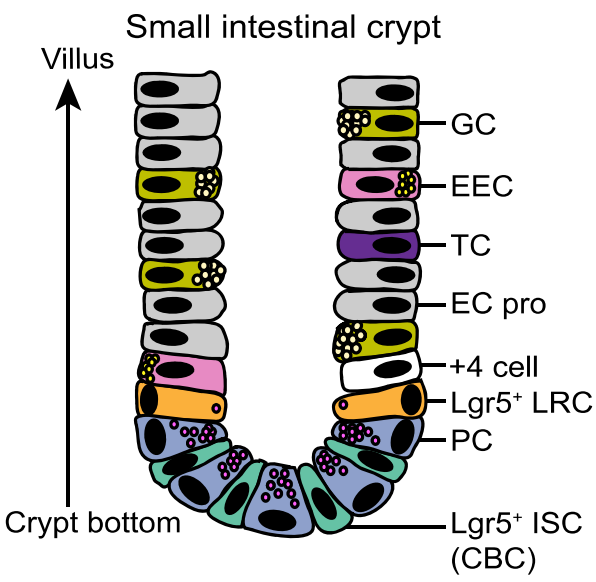

C

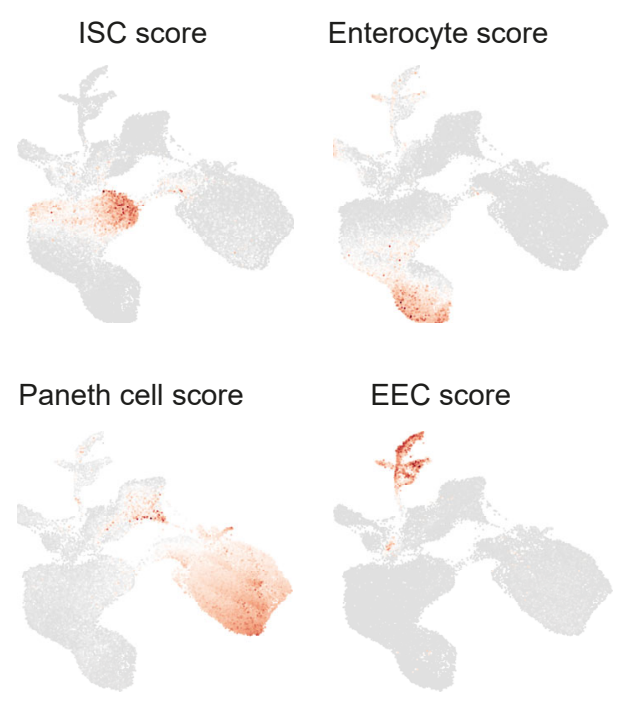

e

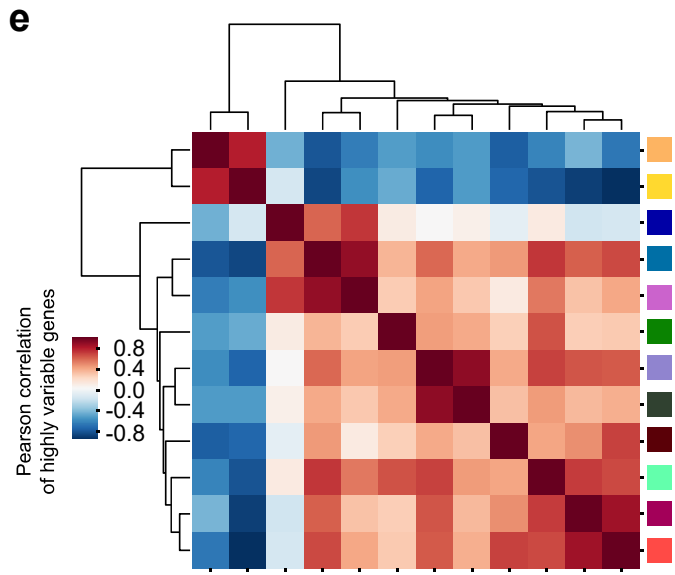

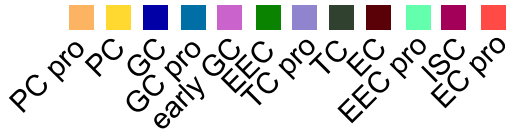

b
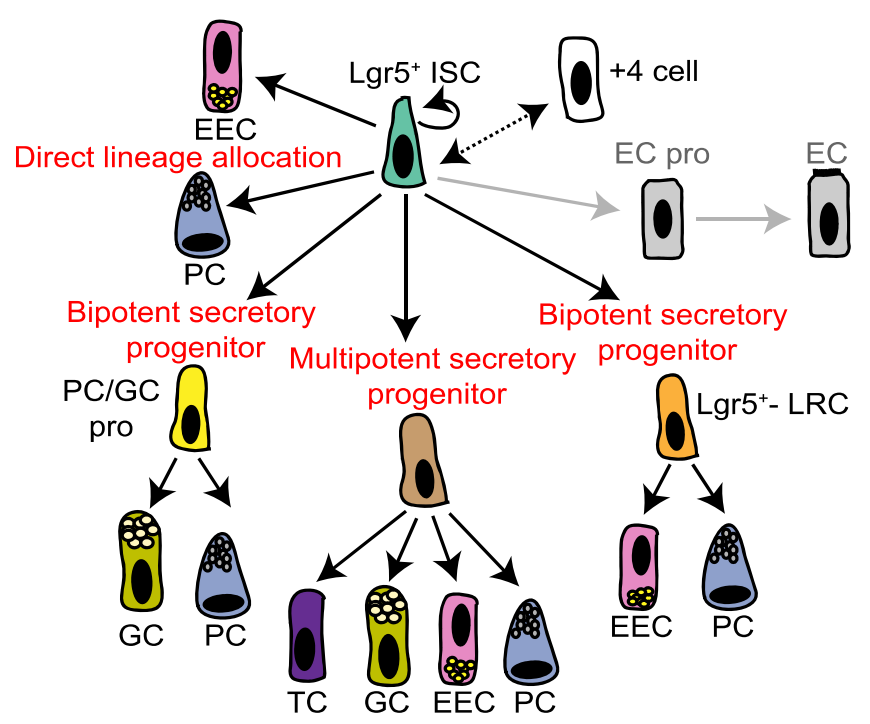

d

Goblet cell score

Tuft cell score

$\mathbf{f}$

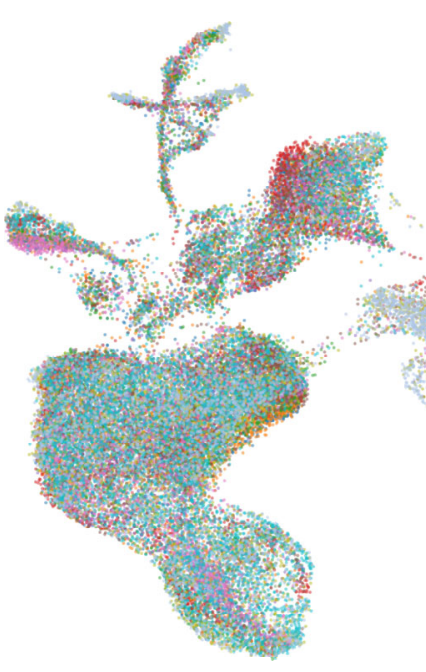

- Control 1

Control-2

Control 3

- $50 \%$ FVF enrichment 1 -50\% FVF enrichment 2

50\% FVF enrichment 3

10\% FVR enrichment 1

-10\% FVR enrichment_2

$50 \%$ FVR enrichment

90\% FVR enrichment
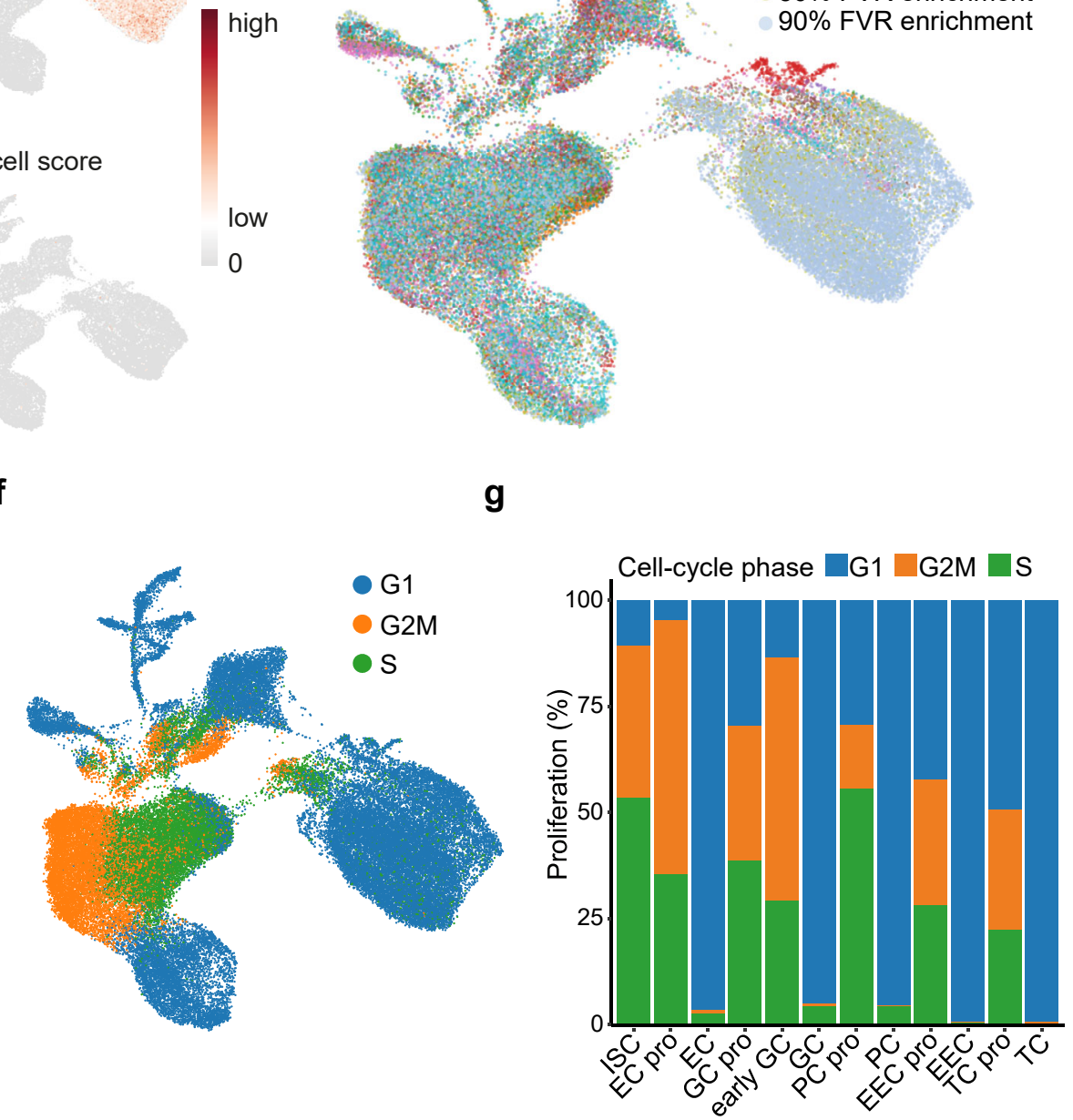
Extended Data Figure 4 Identification of progenitors for each intestinal lineage by scRNAseq.

a, Scheme of a SI crypt depicting all described intestinal epithelial cell types.

b, Overview of current models of secretory and absorptive fate specification in the gut. Wnt $/ \beta$ catenin dependent $\mathrm{Lgr}^{+}$ISCs self-renew or give rise to absorptive or multi-/ bipotent secretory progenitors that further differentiate into enterocytes or Paneth cells (PCs), enteroendocrine cells (EECs), goblet cells, tuft cells, respectively. A direct differentiation route from ISCs has been described for subtypes of Paneth and enteroendocrine cells. +4 reserve stem cells might correspond to $\mathrm{Lgr}^{+}$LRCs.

c, UMAP depicting the gene score of major cell types (Supplementary Table 4).

d, UMAP depicting all samples from control wildtype (no enrichment) and reporter mice (rare lineage enrichment) used in this study.

e, Correlation map showing highly variable genes between progenitor cell types.

f, UMAP depicting the cell-cycle score for each cell.

g, Bar plot showing proliferation score of the progenitor and mature cell types. 
a
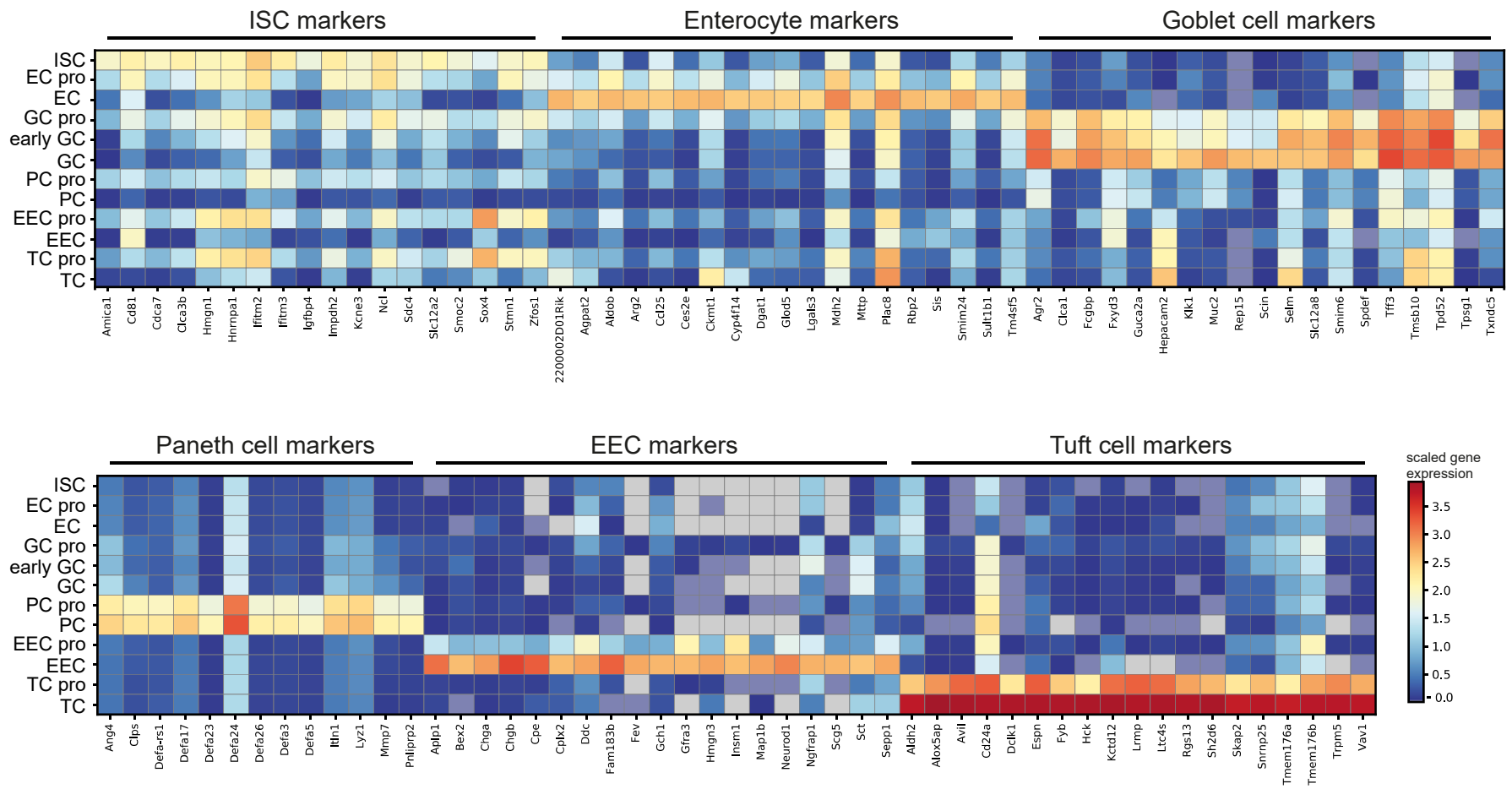

b

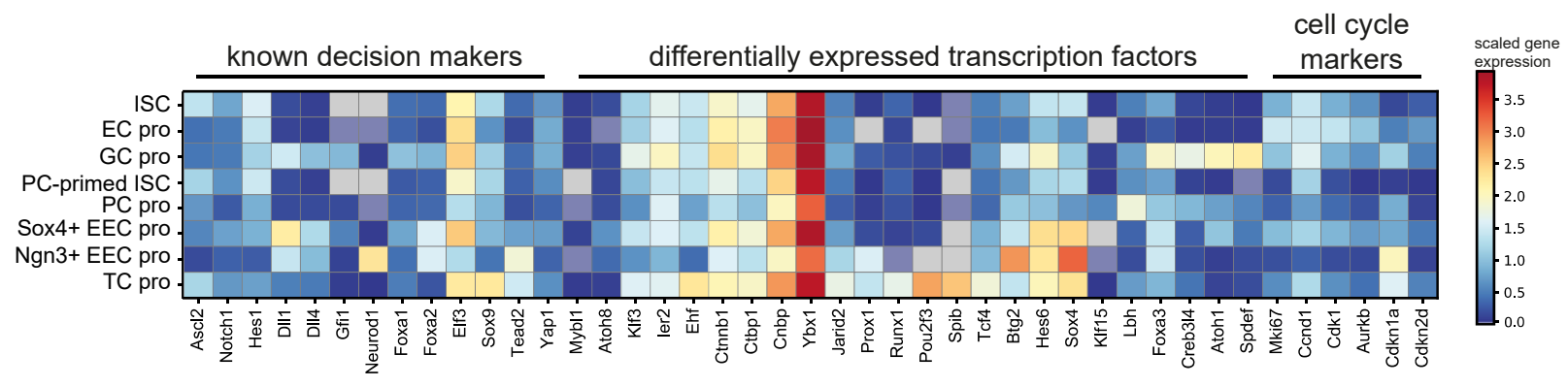

C

\begin{tabular}{|c|c|c|c|c|c|c|c|c|c|c|}
\hline & FGF & EGF & BMP & Notch & & Wnt & $\mathrm{Hh}$ & Hippo Ephrin & Integrin & \\
\hline ISC. & 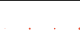 & $\cdots$ & $\ldots$. & . & . &. & . & ... & - & $\begin{array}{l}\text { scaled gene } \\
\text { expression }\end{array}$ \\
\hline EC pro- & & $\cdots$ & $\cdots \cdots$ &.$\quad \bullet$ & & . & &... & . $\bullet$ & $\Pi^{2.00}$ \\
\hline GC pro- & • & $\cdots \cdot$ & $\cdots$ & $\cdots \cdot$ & & . & . & $\ldots .$. & . & $1.50 \bigcirc 100 \%$ \\
\hline PC-primed ISC & & $\cdots$ & $\cdots \cdots$ & $\cdot$ & - & . . & . & $\cdots \cdot \cdot$ & $\bullet$ & $75 \%$ \\
\hline PC pro- & & $\cdots$ & $\ldots \ldots$ & $\cdots$ & . & . . & & $\cdots$ & - & $1.00 \div 50 \%$ \\
\hline Sox4+ EEC pro - & - & $\cdots$ & $\cdots$ & $\cdots \cdot$ & - & $\cdot \cdot$ & . & $\cdots$ & - & \\
\hline Ngn3+ EEC pro- & - & $\cdots$ & . . . . & $\cdots \cdot$ & - & $\cdot \cdot$ & - &.$\cdot \cdot$ & $\bullet$ & 0.50 \\
\hline TC pro- & & $\cdots$ & $\cdots \cdots$ & $\cdots \cdot$ & . & $\cdot \cdot$ & & $\cdots \cdot \cdot \cdot$ & $\cdot \bullet$ & $\perp_{0.00}$ \\
\hline
\end{tabular}

d

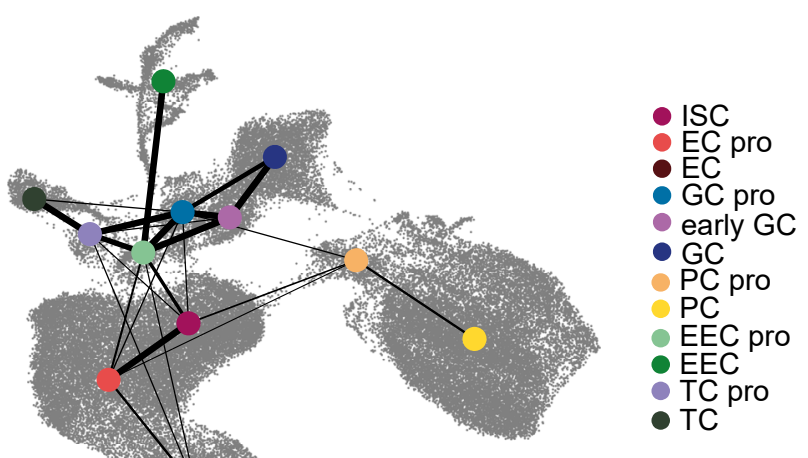


Extended Data Figure 5 Intestinal progenitors are characterized by distinct gene expression patterns.

a, Heatmap depicting the mean expression of marker genes in progenitor and mature cell populations. Distinct marker genes are already expressed in progenitor cells.

b, Heatmap showing differentially expressed transcription factors between progenitors.

c, Heatmap depicting signalling pathways involved in stem-cell maintenance, differentiation and cell positioning. Genes in black show significant expression differences.

d, Abstracted graph of the PAGA model plotted on UMAP, cell-type cluster are reduced to colored dots. Graph abstraction reveals connections between cell types and are depicted as lines. Line thickness describes confidence level of the connection between two cell types (high confidence corresponds to high confidence). Connections below the threshold confidence of 0.05 are not displayed. All progenitor populations connect to ISCs. 
Böttcher et al. Extended Data Figure 6

Pseudotemporal ordering of cells identifies unipotent transition states for EEC and PC lineage characterized by downregulation of stem-cell genes and co-expression of stem-cell and secretory lineage genes, respectively.

a

b
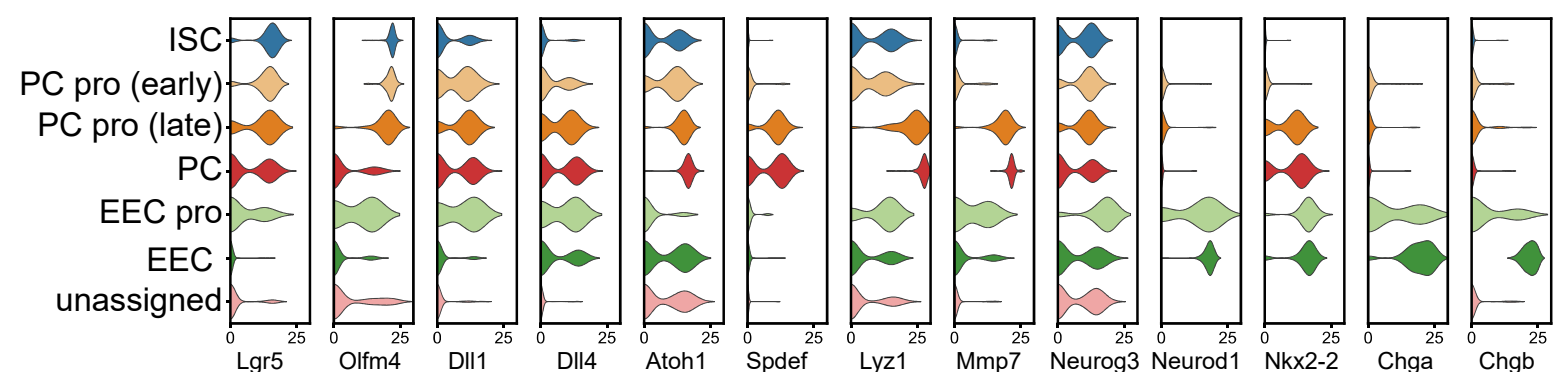

ISC ISC-->PC ISC-->EEC unassigned

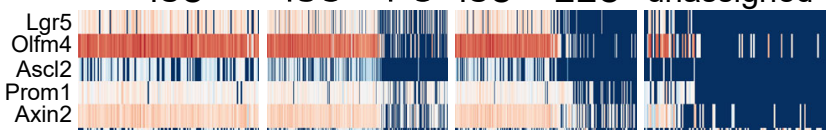

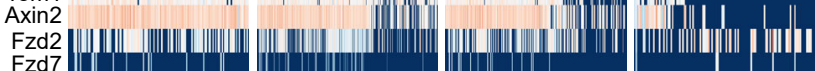

Fzd7 ||

Lrp5

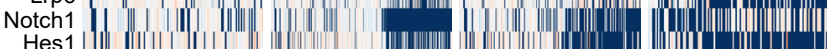

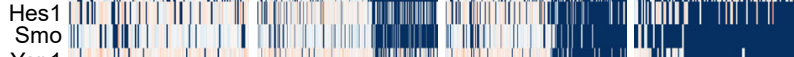

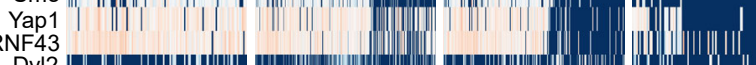

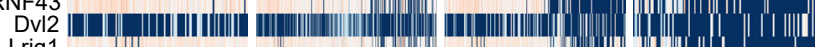

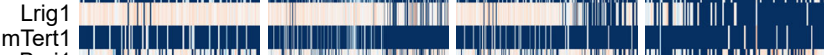

$\underset{\mathrm{Bmi1}}{\mathrm{Bm}}$

Hopx

El

C

PC - cell-cycle markers

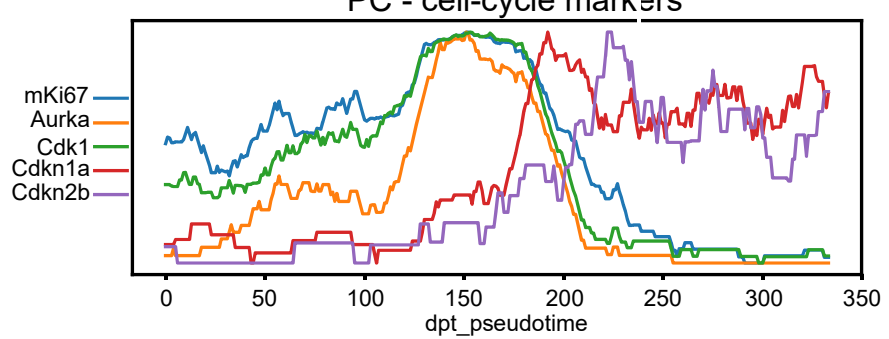

Fabp1
Atoh1

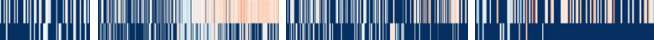

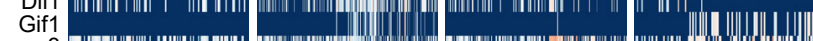

Neurog3

Chga

Chgb

Pax6

Nkx2-2

Sst

Tpg

Sox9

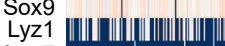

Mmp7

\begin{tabular}{r|l|l|} 
DII4 \\
Muc2
\end{tabular}

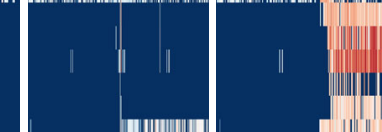

Spdef

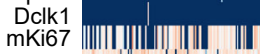

c-myc

Ccnd

Aurka

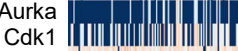
Cdk6

PCNA

(1)

Cdkn2b
EphB3

EphB3

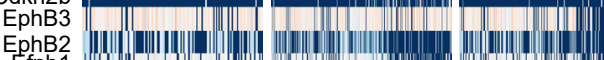

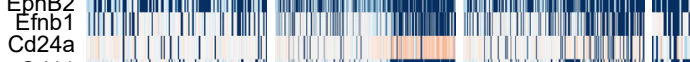

Cd44 || || || || | | ||

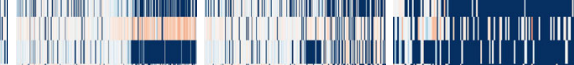

Itgb

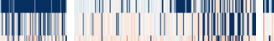

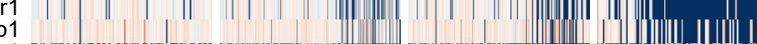

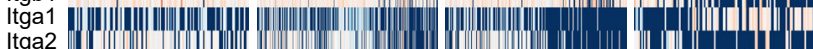

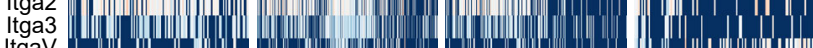

ItgaV L

Itga6

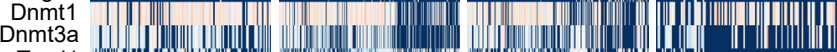

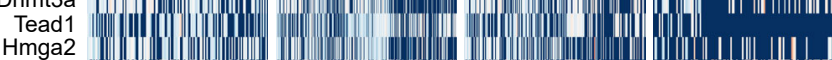

Hea2
Lfng groups_named

group
ISC
PC pro (early)
PC (late)
- PC
EEC pro
EEC
unassigned

state

FVR low

FVR $^{\text {hi }}$

$\mathrm{FVR}^{+} / \mathrm{Lgr}^{\text {hi }}$

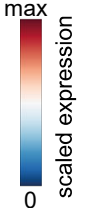

Lgr5 ${ }^{\text {hi }}$

Ngn3-VF

$\mathrm{FVR}^{+} /$Lgr5 $5^{\text {low }}$

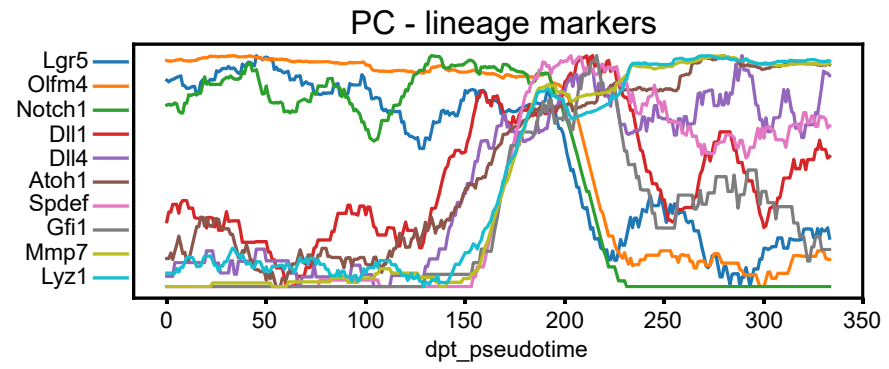

d

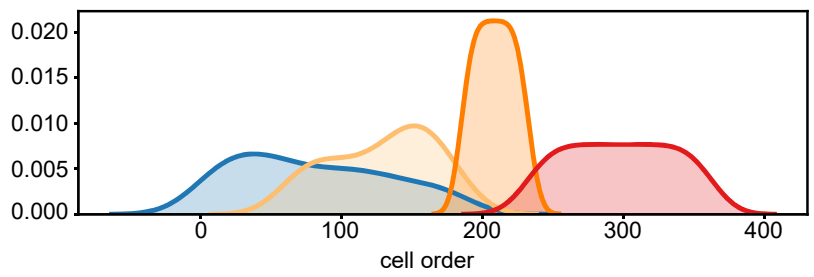

e
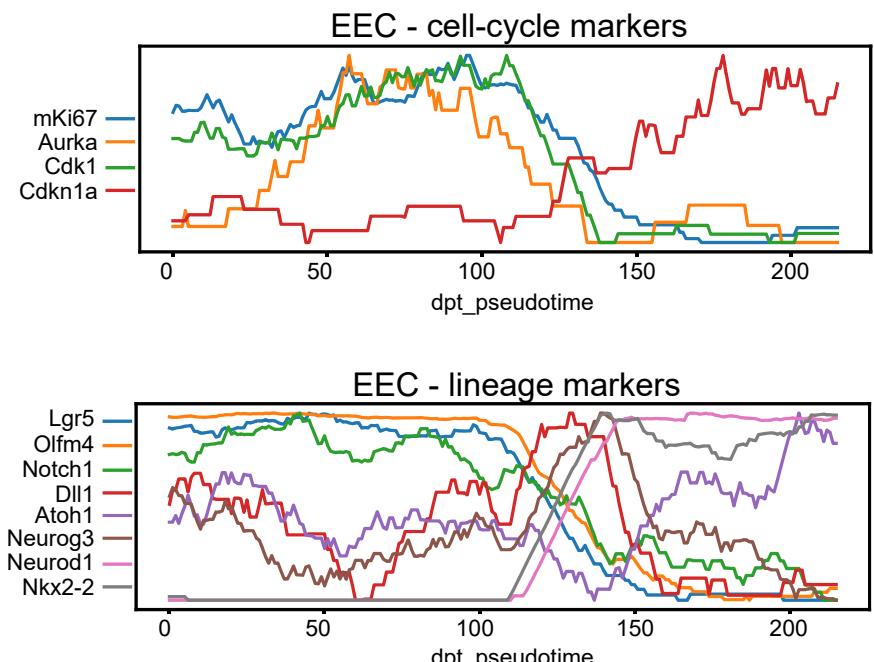

f

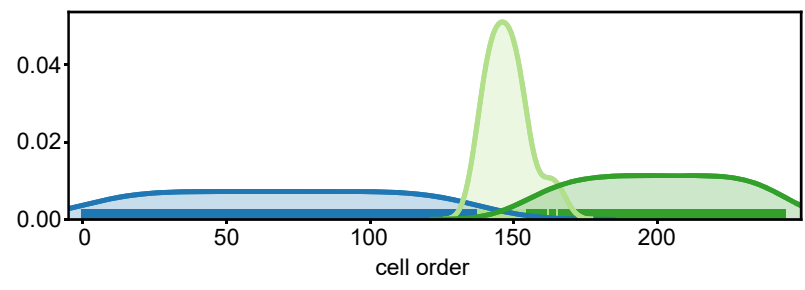


Extended Data Figure 6 Pseudotemporal ordering of cells identifies unipotent transition states for EEC and PC lineage characterized by downregulation of stem cell genes and co-expression of stem cell and secretory lineage genes, respectively.

a, Violin plots showing the distribution of selected marker genes from scqRT-PCR analysis in ISCs, and PC and EEC lineages.

b, Heatmap of gene expression along the lineage-trajectories inferred by PAGA (separated by white vertical bars). Within each trajectory cells are ordered by DPT. Colour bars at the bottom indicate cell-type clusters and FACS states.

c, Expression of cell-cycle (top) and lineage (bottom) marker genes along the PC lineage trajectory. Cells are ordered by DPT. Expression is shown as the running average over 30 cells.

d, Cell density of cell-type clusters along the PC trajectory. Cells are ordered by DPT.

e, Expression of cell-cycle (top) and lineage (bottom) marker genes along the EEC lineage trajectory. Cells are ordered by DPT. Expression is shown as the running average over 30 cells.

f, Cell density of cell type clusters along the EEC trajectory. Cells are ordered by DPT. 


\section{Böttcher et al. Extended Data Figure 7}

Wnt/PCP signalling is activated during differentiation of ISCs towards PCs and EECs.

a

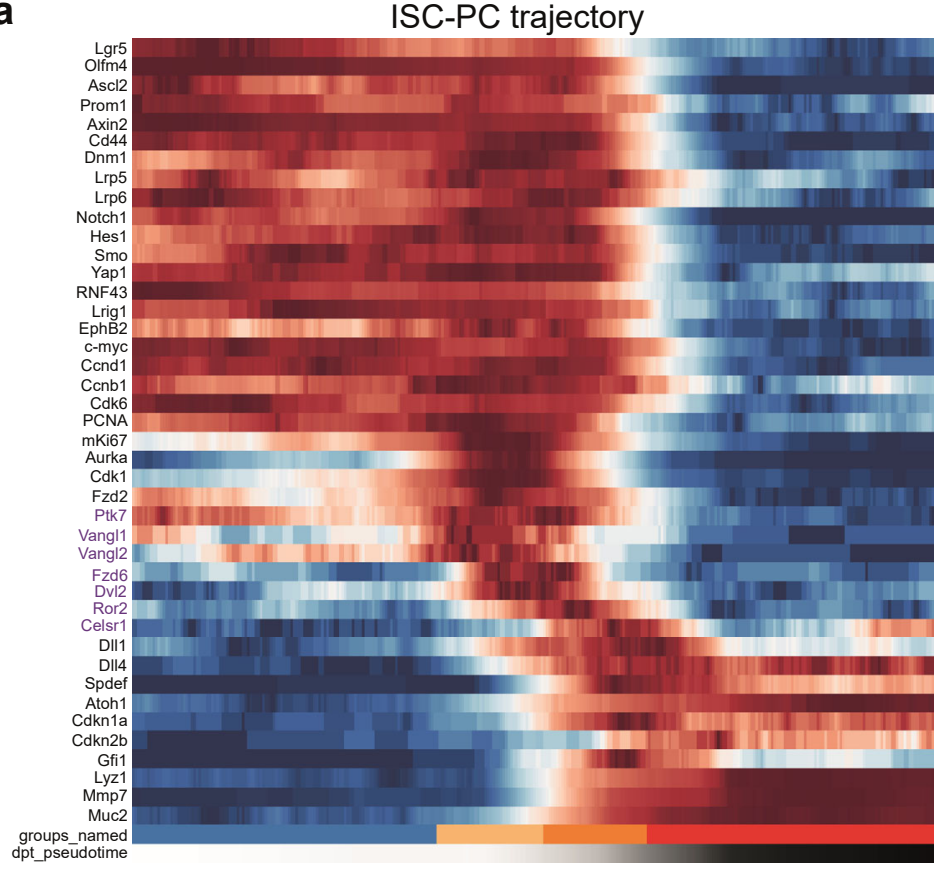

- ISC PC pro (early) PC (late) PC b

ISC-EEC trajectory

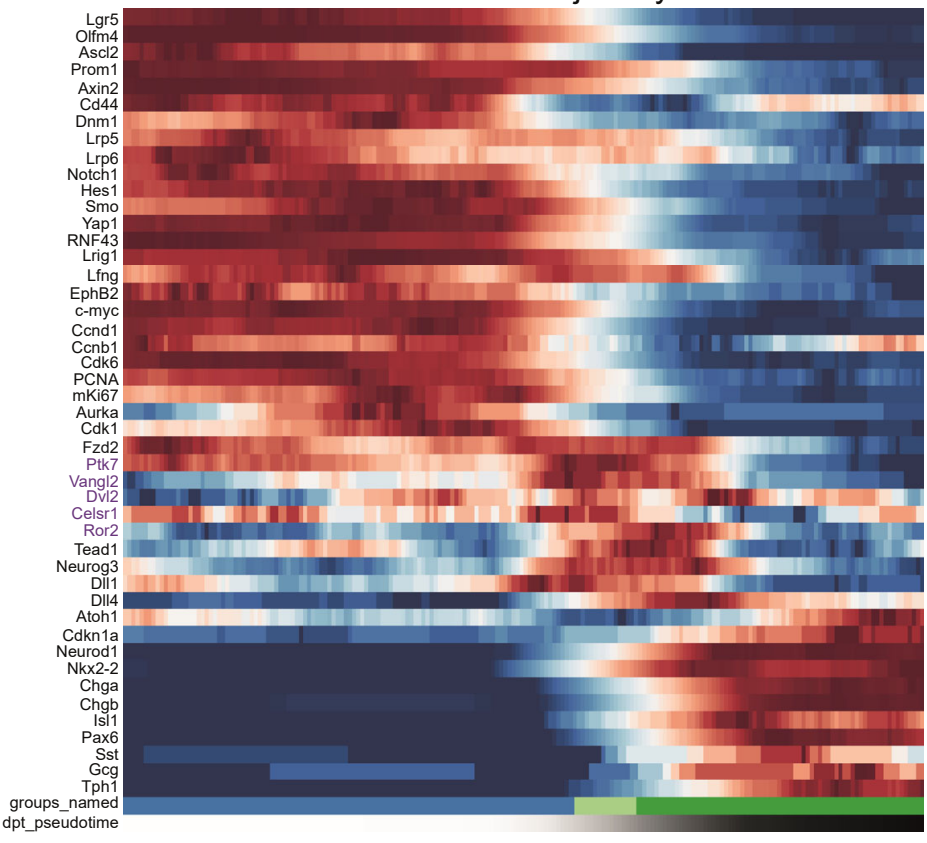

- ISC EEC pro $\bigcirc$ EEC
C

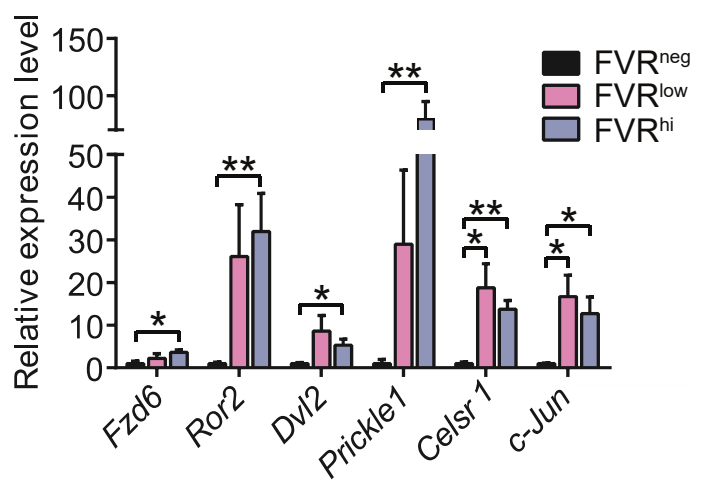

d

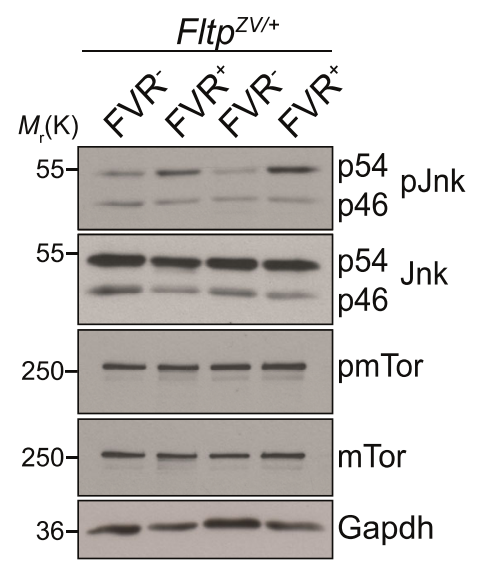

e

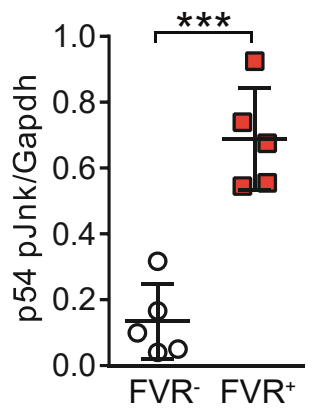


Extended Data Figure $7 \mathrm{Wnt} / \mathrm{PCP}$ signalling is activated during differentiation of ISCs towards PCs and EECs.

a, Heatmap showing gene expression from scqRT-PCR data along the PC lineage trajectory from ISCs to mature PCs. Cell-type clusters are ordered by the inferred PAGA trajectory and cells by DPT. Expression is shown as the running average over 30 cells scaled to the maximum observed level per gene. Bars at the bottom indicate cell type clusters and DPT.

b, Heatmap showing gene expression from scqRT-PCR data along the EEC lineage trajectory from ISCs to mature EECs. Cell-type clusters are ordered by the inferred PAGA trajectory and cells by DPT. Expression is shown as the running average over 30 cells scaled to the maximum observed level per gene. Bars at the bottom indicate cell type clusters and DPT.

c, Expression analysis of Wnt/PCP genes in FVR ${ }^{\text {hi/low/neg }}$ crypt cells obtained from Fltp ${ }^{Z V /+}$ mice by qRT-PCR. $n$ (independent experiments) $=3$. Error bars represent SEM. Two-tailed Student's $t$ test, $* P<0.05, * * P<0.01$.

d, e, Western blot (d) and quantification (e) of active, phosphorylated (p) Jun N-terminal kinase (Jnk) in flow-sorted $\mathrm{FVR}^{+}$and $\mathrm{FVR}^{-}$crypt cells isolated from adult Fltp ${ }^{Z V /+}$ reporter mice. Gapdh, mTor and pmTor are presented as controls. $n$ (independent experiments) $=5$. Error bars represent SD. Two-tailed Student's $t$-test, ${ }^{*} P<0.05$. 


\section{Böttcher et al. Extended Data Figure 8}

Disturbed Wnt/PCP signalling causes alterations in gene expression pattern and numbers of Paneth cells.

\section{a}

Pearson correlation of

highly variable genes

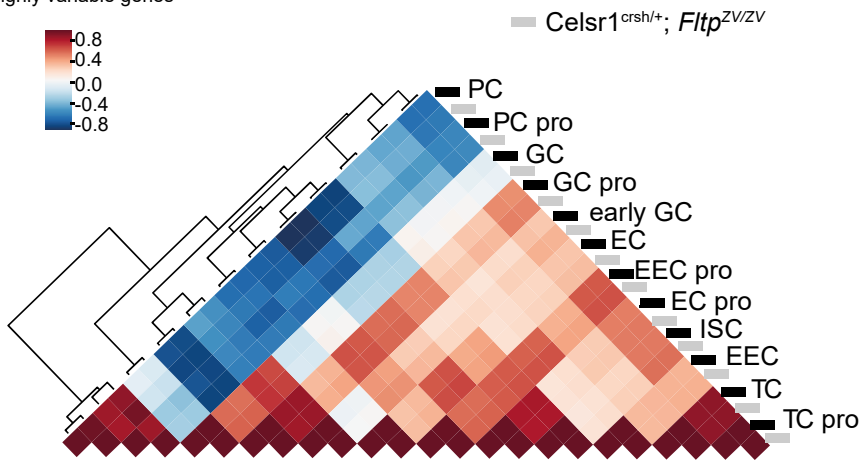

b
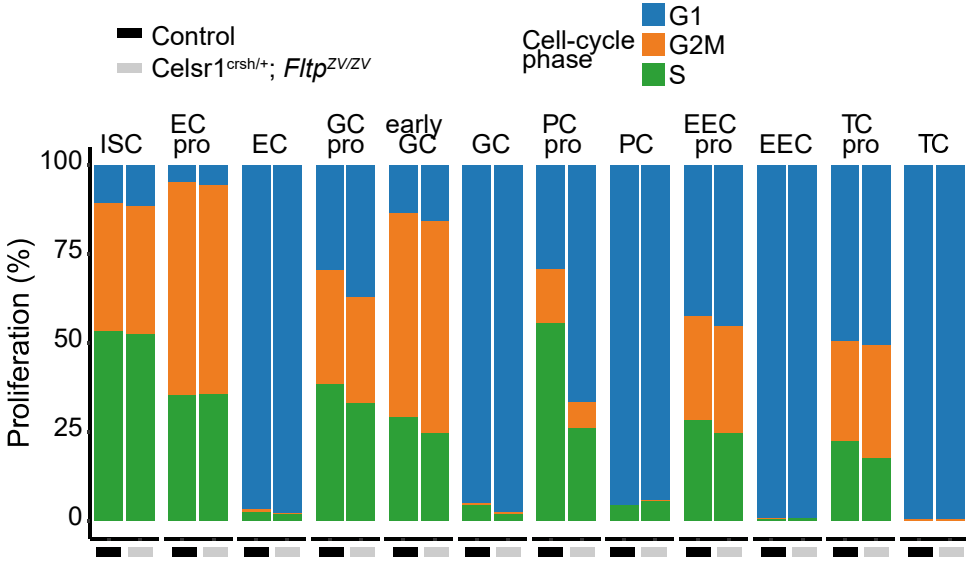

C
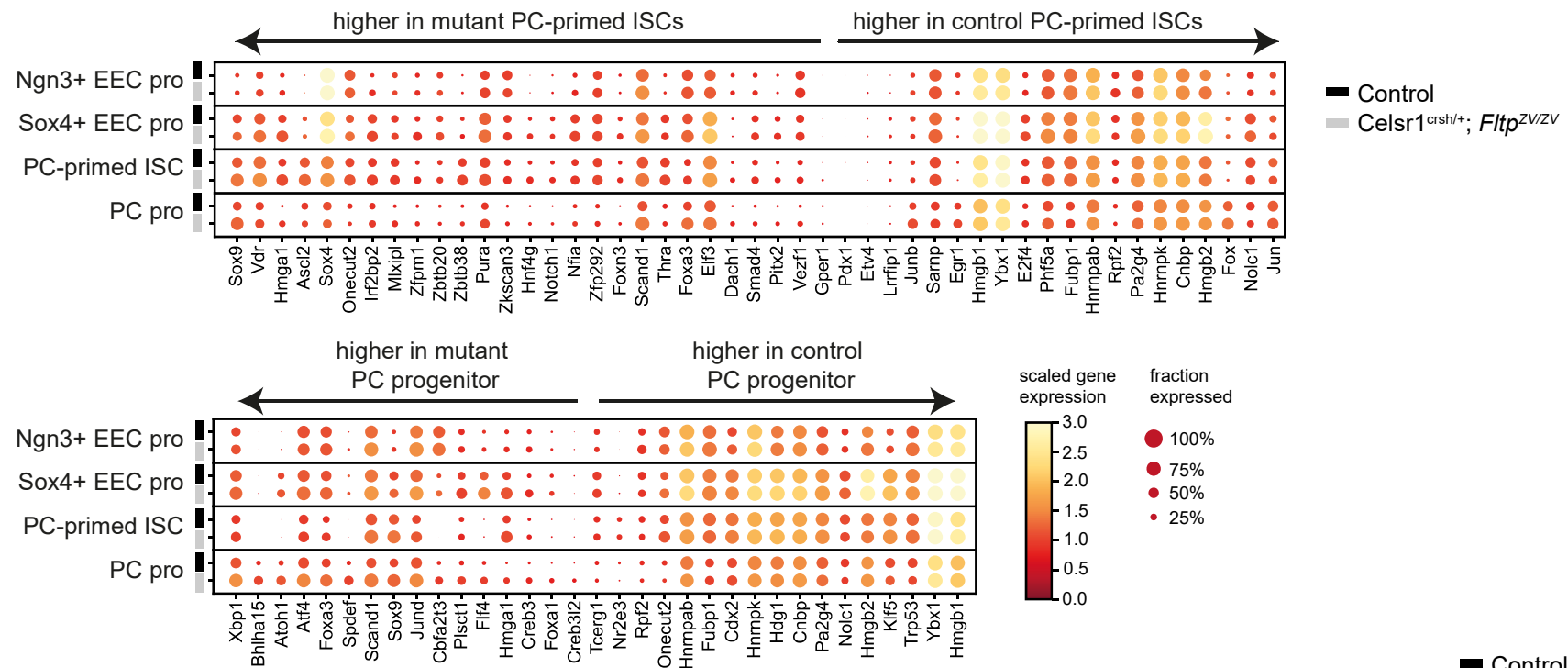

d

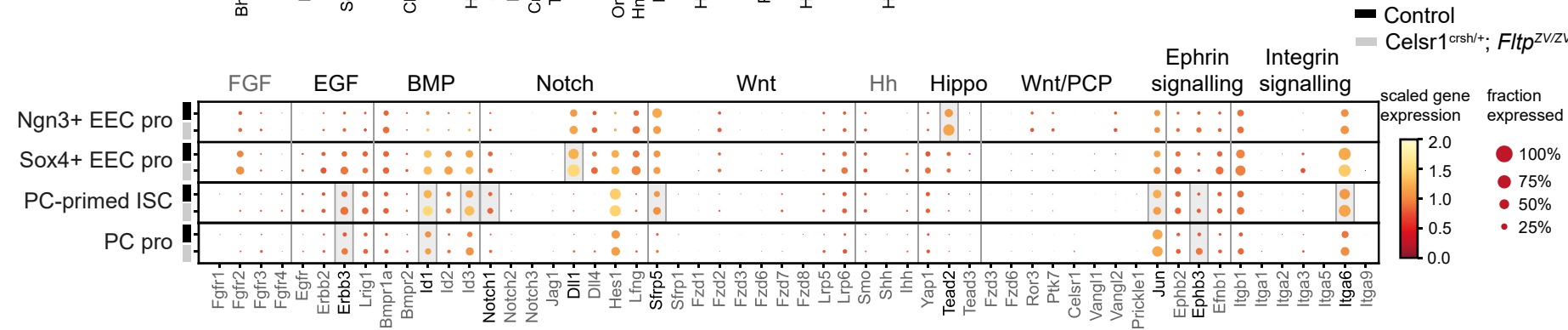

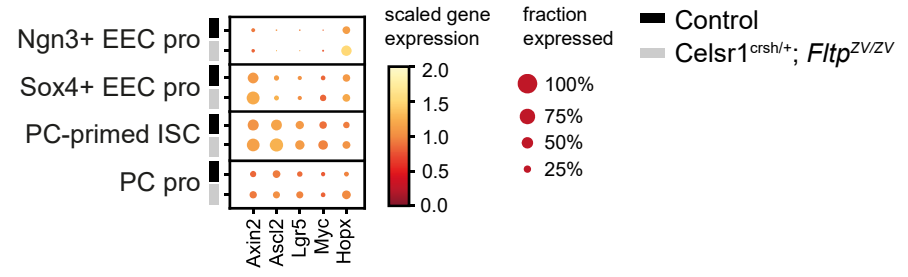

$\mathbf{f}$

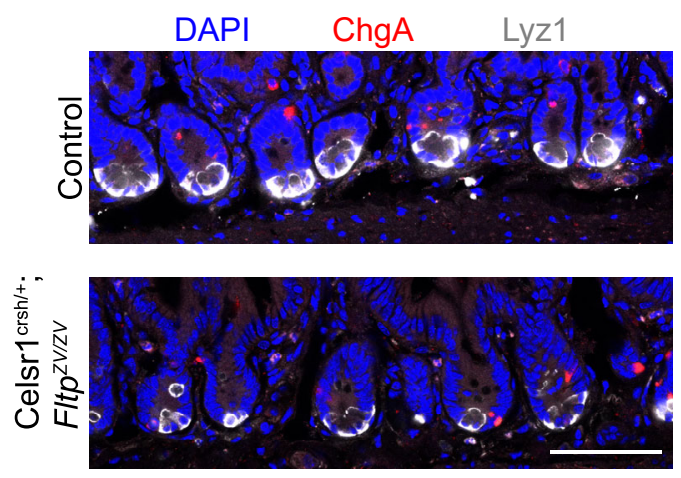

g

- Control

․ Celsr1 ${ }^{\text {crsh } /+} ;$ Fltp $^{z v / z V}$

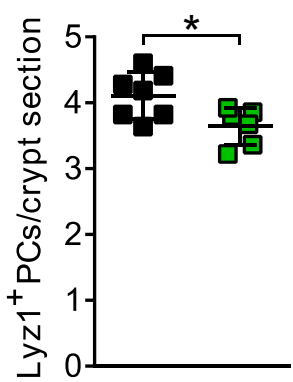

h

- Control

․ Celsr $1^{\text {crsh } /+} ;$ Fltp ${ }^{\text {zv/ZV }}$

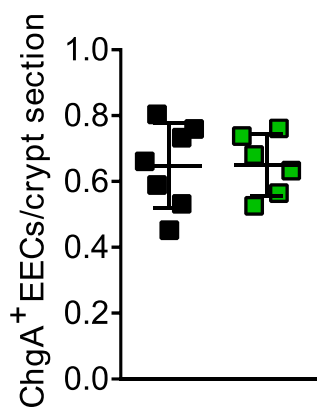


Extended Data Figure 8 Disturbed Wnt/PCP signalling causes alterations in gene expression pattern and numbers of Paneth cells.

a, Heatmap showing Pearson correlation of the highly variable genes in all cell types present in Celsr1 $^{\text {crsh/+}} ;$ Fltp $^{\mathrm{ZV} / \mathrm{ZV}}$ mutant and control samples (see Supplementary Table 2). In all cases, Celsr1 ${ }^{\text {crsh/+}} ;$ Fltp $^{\mathrm{ZV} / \mathrm{ZV}}$ and control samples of the respective cell type correlate the most. $n$ (mice) $=$ 10 for control mice. $n$ (mice) $=4$ for Celsr $1^{\text {crsh/++ }}$; Fltp ${ }^{\mathrm{ZV} / \mathrm{ZV}}$ compound mutant mice.

b, Barplot depicting cell-cycle annotation for all cell types into G1 (non-cycling), S and G2M (proliferating) phase. Paneth progenitors in Celsr $1^{\mathrm{crsh} /+}$; Fltp ${ }^{\mathrm{ZV} / \mathrm{ZV}}$ mutant mice are less proliferative than in control mice. All other cell types are not affected in terms of proliferation. $n($ mice $)=4$ for control mice. $n$ (mice) $=4$ for Celsr $1^{\text {crsh/+ }}$; Fltp ${ }^{\mathrm{ZV} / \mathrm{ZV}}$ compound mutant mice.

c, Heatmap depicting differential expression of transcription factors between Celsr1 ${ }^{\text {crsh/+ }}$; Fltp ${ }^{\mathrm{ZV} / \mathrm{ZV}}$ mutant and control mice and between displayed progenitor groups, ordered by log-fold change in PC-primed ISCs and in PC progenitors. In the EEC progenitors, only Hmgal was significantly different in $\mathrm{Sox}^{+}$early EEC progenitors and none was significantly different in $\mathrm{Ngn}^{+}$ progenitors. Benjamin-Hochberg adjusted p-value $<10^{-5} . n$ (mice) $=10$ for control mice. $n$ (mice) $=4$ for Celsr1 ${ }^{\text {crsh/+}}$; Fltp ${ }^{\mathrm{ZV} / \mathrm{ZV}}$ compound mutant mice.

d, Heatmap depicting differential gene expression in specific signalling pathways. Grey boxes in the dotplot highlight significantly different genes. Benjamin-Hochberg adjusted p-value $<10^{-5} . n$ $($ mice $)=10$ for control mice. $n($ mice $)=4$ for Celsr $1^{\text {crsh/+}}$; Fltp ${ }^{\mathrm{ZV} / \mathrm{ZV}}$ compound mutant mice.

e, Heatmap depicting expression of Wnt target genes. Expression of Axin2, Ascl2 and Lgr5 were significantly different in Celsr1 ${ }^{\text {crsh/+}}$; Fltp ${ }^{\mathrm{ZV} / Z V}$ PC-primed ISCs. Benjamin-Hochberg adjusted pvalue $<10^{-5} . n$ (mice) $=10$ for control mice. $n$ (mice) $=4$ for Celsr $1^{\text {crsh } /+}$; Fltp ${ }^{\mathrm{ZV} / \mathrm{ZV}}$ compound mutant mice.

$\mathbf{f}, \mathbf{g}, \mathbf{h}$, Representative LSM images of duodenal sections stained for ChgA (red, enteroendocrine cells), Lyz1 (white, Paneth cells), DAPI (blue, nucleus) (f) and quantification of Paneth cells (g) and $\mathrm{ChgA}^{+}$EECs $(\mathbf{h})$ in control and Celsr $1^{\text {crsh/+}}$; Fltp ${ }^{\mathrm{ZV} / \mathrm{ZV}}$ compound mutant mice. $n$ (mice) $=6$ for control mice. $n$ (mice) $=7$ for Celsr $1^{\text {crsh/+}}$; Fltp ${ }^{\mathrm{ZV} / \mathrm{ZV}}$ compound mutant mice. Error bars represent SEM. Two-tailed Student's $t$-test, $* P<0.1$. Scale bar, $75 \mu \mathrm{m}$. 